Electron/Photon Verification Calculations

Using MCNP4B

D. P. Gierga

K. J. Adams 



\section{DISCLAIMER}

Portions of this document may be illegible in electronic image products. Images are produced from the best available original document. 


\section{TABLE OF CONTENTS}

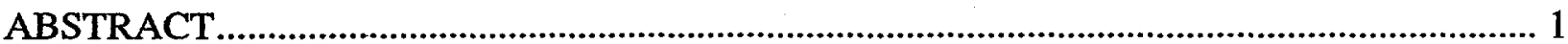

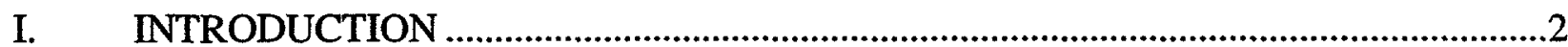

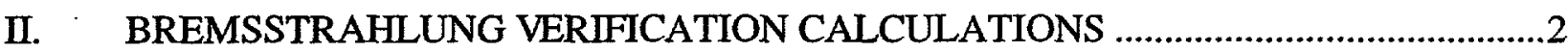

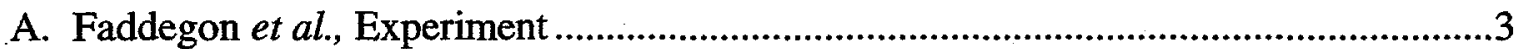

1. Experimental Methods ........................................................................................ 3

2. Monte Carlo Simulation Description ......................................................................... 4

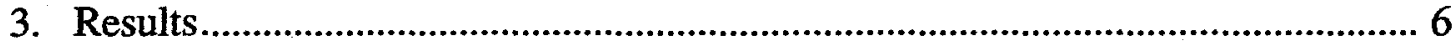

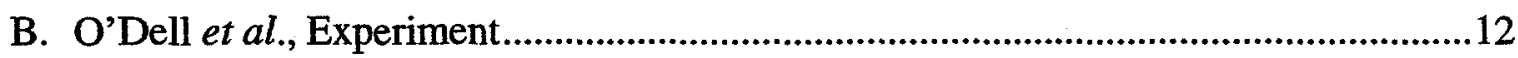

1. Experimental Methods ........................................................................................ 12

2. Monte Carlo Simulation Description ..................................................................... 12

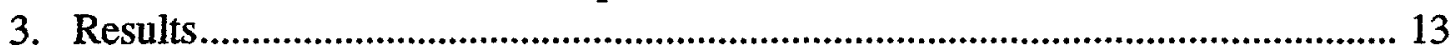

C. Starfelt and Koch Experiment............................................................................14

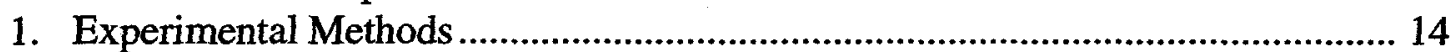

2. Monte Carlo Simulation Description ................................................................. 16

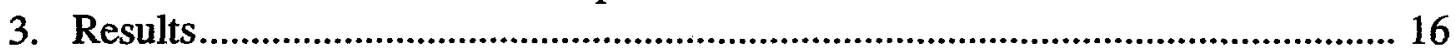

III. ELECTRON TRANSMISSION AND BACKSCATTER …......................................18

A. Ebert et al., Experiment ...................................................................................18

1. Experimental Methods ............................................................................................. 18

2. Monte Carlo Simulation Description ................................................................. 19

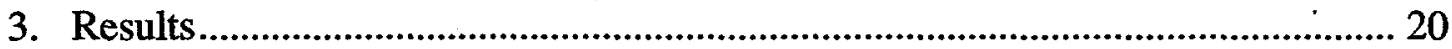

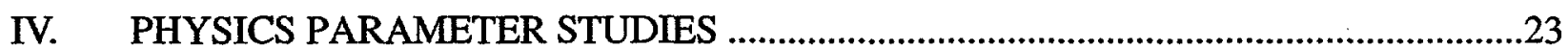

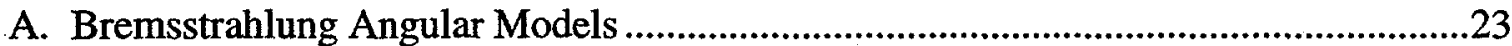

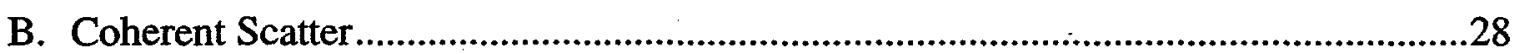

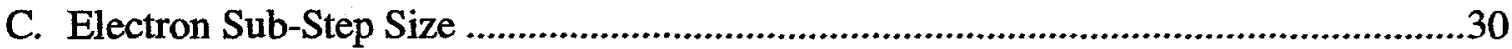

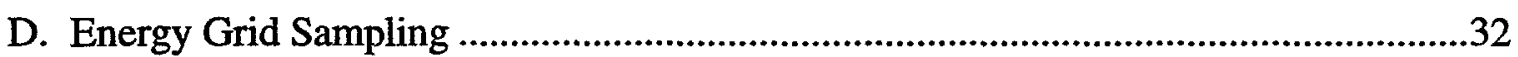

E. Splitting Schemes for Bremsstrahlung Production ......................................................38

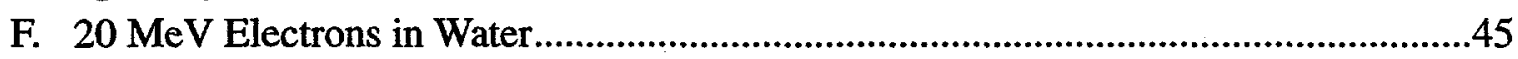

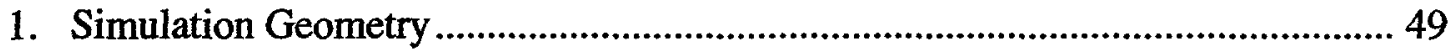

2. Variation of Parameters ................................................................................... 49

3. Problem Tallies ................................................................................................... 51

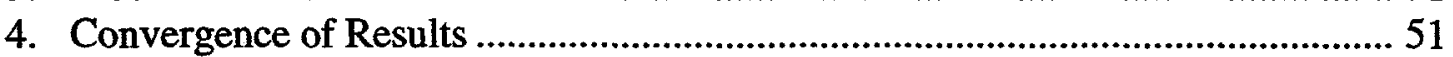

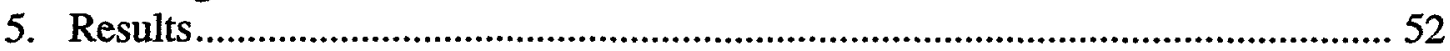

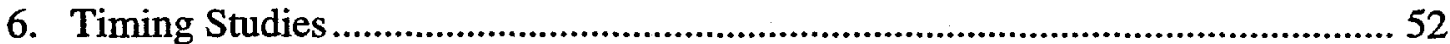

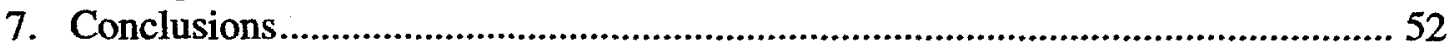

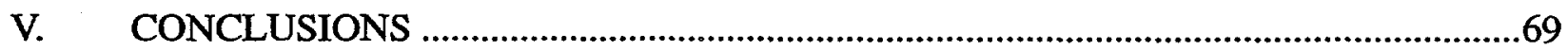

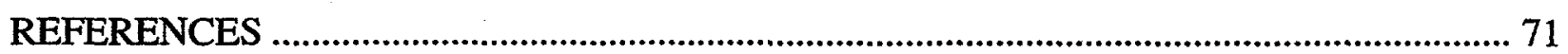

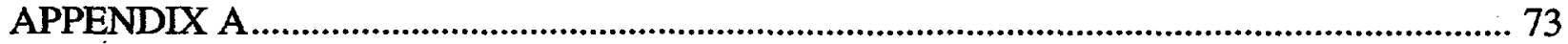

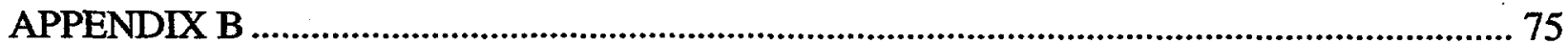


vi 


\section{LIST OF FIGURES}

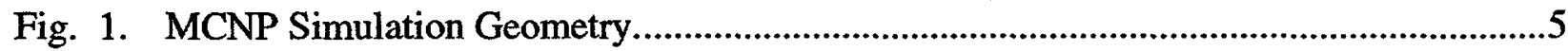

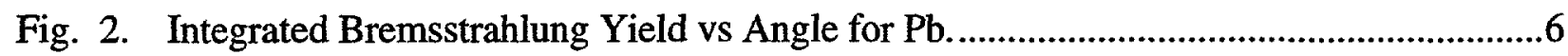

Fig. 3. Integrated Bremsstrahlung Yield vs Angle for Al..........................................................

Fig. 4. Integrated Bremsstrahlung Yield vs Angle for Be..............................................................

Fig. 5. Comparison of experimental and MCNP cell tally bremsstrahlung spectra for aluminum at $10^{\circ}$

Fig. 6. Comparison of experimental and MCNP cell tally bremsstrahlung spectra for beryllium at $10^{\circ}$

Fig. 7. Comparison of experimental and MCNP cell tally bremsstrahlung spectra for lead at $10^{\circ}$.

Fig. 8. Experimental setup for O'Dell et al............................................................................13

Fig. 9. Bremsstrahlung Energy Spectrum, $10.0 \mathrm{MeV}$ electrons incident on $\mathrm{Au} / \mathrm{W}$....................14

Fig. 10. Bremsstrahlung Energy Spectrum, $20.9 \mathrm{MeV}$ electrons incident on $\mathrm{Au} / \mathrm{W}$....................15

Fig. 11. Experimental setup for Starfelt and Koch......................................................................16

Fig. 12. Experimental and calculated bremsstrahlung spectrum at $0^{\circ}$ for $9.66 \mathrm{MeV}$ electrons incident on tungsten.

Fig. 13. Experimental and calculated bremsstrahlung spectrum at $12^{\circ}$ for $9.66 \mathrm{MeV}$ electrons incident on tungsten.

Fig. 14. Geometry for Ebert transmission and backscatter experiments.

Fig. 15. Comparison of transmission coefficients for $10.2 \mathrm{MeV}$ electrons incident on $\mathrm{C}$ foils.

Fig. 16. Comparison of transmission coefficients for $10.2 \mathrm{MeV}$ electrons incident on $\mathrm{Ag}$ foils.

Fig. 17. Comparison of transmission coefficients for $10.2 \mathrm{MeV}$ electrons incident on U foils.

Fig. 18. Integrated bremsstrahlung yield IBAD comparison for lead. 24

Fig. 19. IBAD comparison for aluminum. .26

Fig. 20. IBAD comparison for beryllium .27

Fig. 21. Coherent scatter comparison for lead, detector tally. 
Fig. 22. Increasing ESTEP

Fig. 23. Ratio of calculation to experiment for a few values of ESTEP; Al target.

Fig. 24. Schematic for energy group treatment.

Fig. 25. Bremsstrahlung spectrum at $90^{\circ}$ for default MCNP and DBCN18=1 (nearest bin).......35

Fig. 26. Bremsstrahlung spectrum at $1^{\circ}$ for default MCNP and DBCN18=1 (nearest bin).........36

Fig. 27. Comparison of transmission coefficients for $10.2 \mathrm{MeV}$ electrons incident on $\mathrm{C}$ foils.

Fig. 28. Comparison of transmission coefficients for $10.2 \mathrm{MeV}$ electrons incident on $\mathrm{Ag}$ foils.

Fig. 29. Comparison of transmission coefficients for $10.2 \mathrm{MeV}$ electrons incident on $\mathrm{U}$ foils.

Fig. 30. A comparison of the Starfelt and Koch bremsstrahlung spectra for default (BNUM=1) and standard $\mathrm{BNUM}=20$ variance reduction for the $0^{\circ}$ cell tally. .40

Fig. 31. A comparison of the Starfelt and Koch bremsstrahlung spectra for default (BNUM=1) and standard $\mathrm{BNUM}=20$ variance reduction for the $12^{\circ}$ cell tally. .40

Fig. 32. A comparison of the $\mathrm{Pb}$ bremsstrahlung spectra for default (BNUM=1) and standard $\mathrm{BNUM}=20$ variance reduction for the $0^{\circ}$ cell tally

Fig. 33. A comparison of the $\mathrm{Pb}$ bremsstrahlung spectra for standard and modified BNUM sampling $(B N U M=20)$ variance reduction for the $0^{\circ}$ cell tally.

Fig. 34. A comparison of the $\mathrm{Pb}$ bremsstrahlung spectra for default (BNUM=1) and standard $\mathrm{BNUM}=20$ variance reduction for the $2^{\circ}$ cell tally

Fig. 35. A comparison of the $\mathrm{Pb}$ bremsstrahlung spectra for standard and modified BNUM sampling $(B N U M=20)$ variance reduction for the $2^{\circ}$ cell tally.

Fig. 36. Ratio of modified FOM to standard FOM vs BNUM for photon flux cell tallies.

Fig. 37. Ratio of modified FOM to standard FOM vs BNUM for photon flux detector tallies...45

Fig. 38. Ratio of FOM $\mathrm{F}_{\mathrm{i}}$ to $\mathrm{FOM}_{1}$ vs BNUM for standard splitting; cell tallies. .46

Fig. 39. Ratio of $\mathrm{FOM}_{\mathbf{i}}$ to $\mathrm{FOM}_{1}$ vs BNUM for modified splitting; cell tallies. .46

Fig. 40. Ratio of $\mathrm{FOM}_{\mathrm{i}}$ to $\mathrm{FOM}_{1}$ vs BNUM for standard splitting; detector tallies. .47

Fig. 41. Ratio of $\mathrm{FOM}_{\mathrm{i}}$ to $\mathrm{FOM}_{1}$ vs BNUM for standard splitting sampling; detector tallies. ....47

Fig. 42. FOM vs BNUM for photon current exiting the downstream face of the target in the forward direction. 
Fig. 43. FOM vs BNUM for photon flux tally of photons crossing the downstream face of the target

Fig. 44. Depth Dose Geometry

Fig. 45. The solid line indicates default settings. The dashed line indicates the same settings, except that there are no knock-on electrons. In both cases, the problem includes photons and electrons.

Fig. 46. The solid line indicates default settings. The dashed line indicates the same settings, except that there are no knock-on electrons. For the default case, the problem includes photons and electrons, whereas only electrons are included in the other case.

Fig. 47. The solid line indicates default settings. The dashed line indicates the same settings, except that there are no knock-on electrons, and the ITS energy grid is used. In both cases, the problem includes photons and electrons.

Fig. 48. The solid line indicates default settings. The dashed line indicates the same settings, except that there are no knock-on electrons, and the ITS energy grid is used. For the default case, the problem includes photons and electrons, whereas only electrons are included in the other case.

Fig. 49. The solid line indicates all default settings. The dashed line indicates all default settings, except the problem only includes electrons.

Fig. 50. Both runs have default settings, except for ESTEP. The solid line is estep=3 (default), whereas the dashed line is ESTEP $=15$.

Fig. 51. The solid line indicates default settings. The dashed line indicates the same settings, except that the ITS energy grid is used. In both cases, the problem includes photons and electrons.

Fig. 52. The solid line indicates default settings. The dashed line indicates the same settings, except that the ITS energy grid is used. For the default case, the problem includes photons and electrons, whereas only electrons are included in the other case.

Fig. 53. The solid line indicates default settings. The dashed line indicates the same settings, except that there is no straggling, i.e., CSDA energy loss, and no knock-on electrons. In both cases, the problem includes photons and electrons.

Fig. 54. The solid line indicates default settings. The dashed line indicates the same settings, except that there is CSDA energy loss, and no knock-on electrons. For the default case, the problem includes photons and electrons, whereas only electrons are included in the other case.

Fig. 55. The solid line indicates default settings. The dashed line indicates the same settings, except that there is CSDA energy loss, the ITS energy grid is used, and there are no knock-on electrons. In both cases, the problem includes photons and electrons. 
Fig. 56. The solid line indicates default settings. The dashed line indicates the same settings, except that there is CSDA energy loss, the ITS energy grid is used, and no knock-on electrons. For the default case, the problem includes photons and electrons, whereas only electrons are included in the other case.

Fig. 57. The solid line indicates default settings. The dashed line indicates the same settings, except that there is CSDA energy loss. In both cases, the problem includes photons and electrons.

Fig. 58. The solid line indicates default settings. The dashed line indicates the same settings, except that there is CSDA energy loss. For the default case, the problem includes photons and electrons, whereas only electrons are included in the other case.

Fig. 59. The solid line indicates default settings. The dashed line indicates the same settings, except that there is CSDA energy loss, and the ITS energy grid is used. In both cases, the problem includes photons and electrons.

Fig. 60. The solid line indicates default settings. The dashed line indicates the same settings, except that there is CSDA energy loss, and the ITS energy grid is used. For the default case, the problem includes photons and electrons, whereas only electrons are included in the other case.

Fig. 61. The solid line indicates default settings. The dashed line indicates the same settings, except that electrons do not produce photons, and there are no knock-on electrons. In both cases, the problem includes photons and electrons.

Fig. 62. The solid line indicates default settings. The dashed line indicates the same settings, except that electrons do not produce photons, and there are no knock-on electrons. For the default case, the problem includes photons and electrons, whereas only electrons are included in the other case.

Fig. 63. The solid line indicates default settings. The dashed line indicates the same settings, except that electrons do not produce photons, there are no knock-on electrons, and the ITS energy grid is used. In both cases, the problem includes photons and electrons....62

Fig. 64. The solid line indicates default settings. The dashed line indicates the same settings, except that electrons do not produce photons, there are no knock-on electrons, and the ITS energy grid is used. For the default case, the problem includes photons and electrons, whereas only electrons are included in the other case.

Fig. 65. The solid line indicates default settings. The dashed line indicates the same settings, except that electrons do not produce photons. In both cases, the problem includes photons and electrons.

Fig. 66. The solid line indicates default settings. The dashed line indicates the same settings, except that electrons do not produce photons. For the default case, the problem includes photons and electrons, whereas only electrons are included in the other case. 
Fig. 67. The solid line indicates default settings. The dashed line indicates the same settings, except that electrons do not produce photons, and the ITS energy grid is used. In both cases, the problem includes photons and electrons.

Fig. 68. The solid line indicates default settings. The dashed line indicates the same settings, except that electrons do not produce photons, and the ITS energy grid is used. For the default case, the problem includes photons and electrons, whereas only electrons are included in the other case.

Fig. 69. The solid line indicates default settings. The dashed line indicates the same settings, except that electrons do not produce photons, there are no knock-on electrons, and CSDA energy loss. In both cases, the problem includes photons and electrons.

Fig. 70. The solid line indicates default settings. The dashed line indicates the same settings, except that electrons do not produce photons, there are no knock-on electrons, and CSDA energy loss. For the default case, the problem includes photons and electrons, whereas only electrons are included in the other case.

Fig. 71. The solid line indicates default settings. The dashed line indicates that electrons do not produce photons, there are no knock-on electrons, the ITS energy grid is used, and CSDA energy loss. In both cases, the problem includes photons and electrons.

Fig. 72. The solid line indicates default settings. The dashed line indicates that electrons do not produce photons, there are no knock-on electrons, the ITS energy grid is used, and CSDA energy loss. For the default case, the problem includes photons and electrons, whereas only electrons are included in the other case.

Fig. 73. The solid line indicates default settings. The dashed line indicates the same settings, except that electrons do not produce photons, and there is CSDA energy loss. In both cases, the problem includes photons and electrons.

Fig. 74. The solid line indicates default settings. The dashed line indicates the same settings, except that electrons do not produce photons, and there is CSDA energy loss. For the default case, the problem includes photons and electrons, whereas only electrons are included in the other case.

Fig. 75. The solid line indicates default settings. The dashed line indicates the same settings, except that electrons do not produce photons, the ITS energy grid is used, and there is CSDA energy loss. In both cases, the problem includes photons and electrons.

Fig. 76. The solid line indicates default settings. The dashed line indicates the same settings, except that electrons do not produce photons, the ITS energy grid is used, and there is CSDA energy loss. For the default case, the problem includes photons and electrons, whereas only electrons are included in the other case. 


\section{LIST OF TABLES}

TABLE I: $\quad$ INTEGRATED BREMSSTRAHLUNG YIELD VS ANGLE FOR PB ....................7

TABLE II: INTEGRATED BREMSSTRAHLUNG YIELD VS ANGLE FOR AL .....................8

TABLE III: INTEGRATED BREMSSTRAHLUNG YIELD VS ANGLE FOR BE ......................9

TABLE IV: $\quad$ BREMSSTRAHLUNG SPECTRA MEAN ENERGIES .......................................12

TABLE V: INTEGRATED YIELDS FOR AU/W FOR O'DELL ........................................15

TABLE VI: INTEGRATED YIELDS FOR TUNGSTEN FOR STARFELT AND KOCH.........18

TABLE VII: $\quad$ ELECTRON SATURATION BACKSCATTER COMPARISON ............................22

TABLE VIII: PB INTEGRATED BREMSSTRAHLUNG YIELD, IBAD COMPARISON..........25

TABLE IX: AL INTEGRATED BREMSSTRAHLUNG YIELD, IBAD COMPARISON ........25

TABLE X: $\quad$ BE INTEGRATED BREMSSTRAHLUNG YIELD, IBAD COMPARISON .........26

TABLE XI: $\quad$ PB INTEGRATED BREMSSTRAHLUNG YIELD, MCNP4A IBAD

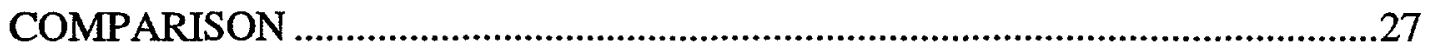

TABLE XII: AL INTEGRATED BREMSSTRAHLUNG YIELD, MCNP4A IBAD

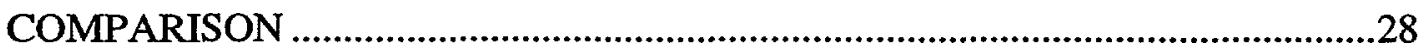

TABLE XIII: PB INTEGRATED BREMSSTRAHLUNG YIELD, COHERENT SCATTER

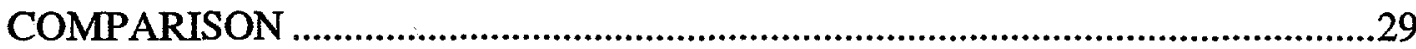

TABLE XIV: MCNP4A ESTEP COMPARISON FOR AL …………..........................................31

TABLE XV: $\quad$ MCNP4B ESTEP COMPARISON FOR AL …….................................................

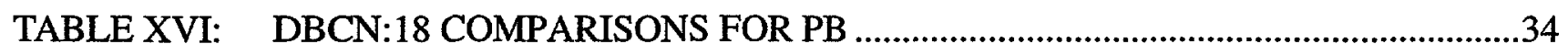

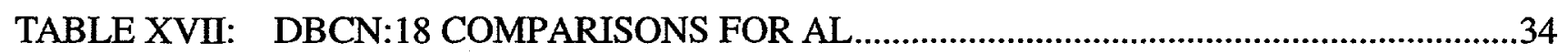

TABLE XVIII: ELECTRON SATURATION BACKSCATTER COMPARISON ..........................38

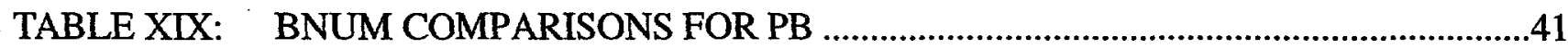

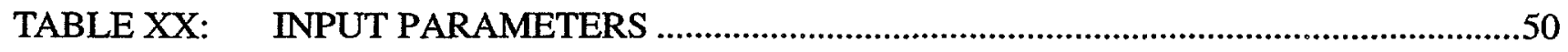

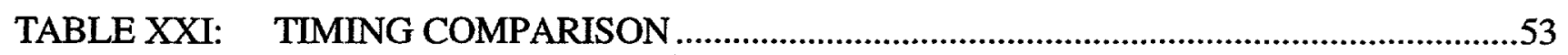




\title{
ELECTRON/PHOTON VERIFICATION CALCULATIONS USING MCNP4B
}

\author{
by
}

\author{
D. P. Gierga and K. J. Adams
}

\begin{abstract}
MCNP4B $^{\text {TM }}$ was released in February 1997 with significant enhancements to electron/photon transport methods. These enhancements have been verified against a wide range of published electron/photon experiments, spanning high energy bremsstrahlung production to electron transmission and reflection. The impact of several MCNP tally options and physics parameters was explored in detail. The agreement between experiment and simulation was usually within two standard deviations of the experimental and calculational errors. Furthermore, sub-step artifacts for bremsstrahlung production were shown to be mitigated. A detailed suite of electron depth dose calculations in water is also presented. Areas for future code development have also been explored and include the dependence of cell and detector tallies on different bremsstrahlung angular models and alternative variance reduction splitting schemes for bremsstrahlung production.
\end{abstract}

MCNP is a trademark of the Regents of the University of California, Los Alamos National Laboratory. 


\section{INTRODUCTION}

MCNP4B was released in February 1997 with significant enhancements to electron/photon transport methods. ${ }^{1}$ These enhancements have been verified against a wide range of published electron/photon experiments, spanning high-energy bremsstrahlung production to electron transmission and reflection. Three sets of bremsstrahlung experiments were simulated. The first verification calculations for bremsstrahlung production used the experimental results of Faddegon for $15 \mathrm{MeV}$ electrons incident on lead, aluminum, and beryllium targets. The calculated integrated bremsstrahlung yields, the bremsstrahlung energy spectra, and the mean energy of the bremsstrahlung beam were compared with experiment. The impact of several MCNP tally options and physics parameters was explored in detail. The second was the experiment of O'Dell which measured the bremsstrahlung spectra from 10 and $20.9 \mathrm{MeV}$ electrons incident on a gold/ tungsten target. The final set was a comparison of relative experimental spectra with calculated results for $9.66 \mathrm{MeV}$ electrons incident on tungsten based on the experiment of Starfelt and Koch. The transmission experiments of Ebert were also studied, including comparisons of transmission coefficients for $10.2 \mathrm{MeV}$ electrons incident on carbon, silver, and uranium foils. Backscatter . coefficients for electrons of a range of energies were also compared. The agreement between experiment and simulation was usually within two standard deviations of the experimental and calculational errors. Furthermore, sub-step artifacts for bremsstrahlung production were shown to be mitigated. A detailed suite of electron depth dose calculations in water is also presented. Areas for future code development have also been explored and include the dependence of cell and detector tallies on different bremsstrahlung angular models and alternative variance reduction splitting schemes for bremsstrahlung production.

\section{BREMSSTRAHLUNG VERIFICATION CALCULATIONS}

This portion of the report describes a set of thick-target bremsstrahlung calculations. The targets are "thick" in that their thickness is comparable to an electron range; the targets are thin to photons. Previous studies on these data have been performed using EGS, ${ }^{2,3}$ ITS, ${ }^{4}$ and MCNP4A. ${ }^{5}$ MCNP4B simulations of three sets of experiments are described. They are the absolute thick-target bremsstrahlung measurements of Faddegon et al., ${ }^{6,7}$ and O'Dell et al., ${ }^{8}$ as well as the relative bremsstrahlung measurements of Starfelt and Koch. ${ }^{9}$ 


\section{A. Faddegon et al., Experiment}

1. Experimental Methods. The most detailed set of the three bremsstrahlung calculations described in this report were the MCNP simulations of the experiments of Faddegon et al. ${ }^{6,7}$ These experiments provided bremsstrahlung spectra and integrated yields from thick targets of $\mathrm{Be}, \mathrm{Al}$, and $\mathrm{Pb}$ at angles of $0^{\circ}, 1^{\circ}, 2^{\circ}, 4^{\circ}, 10^{\circ}, 30^{\circ}, 60^{\circ}$, and $90^{\circ}$ relative to the beam axis for electrons of $15 \mathrm{MeV}$ incident energy. The spectra are absolute in the sense that they are in units of photons per incident electron.

The bremsstrahlung yield, as defined by Faddegon, is the number of photons of energy $E$ per unit energy from the target which reach a given point $P$ in a vacuum per unit solid angle per electron incident on the target. The solid angle is defined from the point of intersection of the electron beam axis with the upstream surface of the target. The differential bremsstrahlung yield can be written

$$
\frac{d S}{d E}=\frac{1}{N_{e}} \frac{d^{2} N_{\gamma}(E)}{d E d \Omega}
$$

where the derivative term on the right hand side of the Eq. (1) is the number of photons with energy between $E$ and $E+d E$ which exit the target and reach point $P$ per unit solid angle, and $N_{e}$ is the number of incident electrons. The integrated bremsstrahlung yield can be written

$$
S_{E_{0}}=\int_{E_{0}}^{E_{\max }} \frac{d S}{d E} d E
$$

where $E_{0}$ is the low energy cutoff and $E_{\max }$ is the maximum photon energy in the spectrum, which is equal to the incident electron energy. Both the experiment and simulations used a low energy cutoff of $145 \mathrm{keV}$.

The electron beam passed through a thin Ti exit window $(0.013 \mathrm{~cm})$ and a Si beam monitoring detector $(0.01 \mathrm{~cm})$ prior to impinging on the target chamber. The targets were $\mathrm{Pb}(9.13 \mathrm{~g} /$ $\mathrm{cm}^{2}$ thick, $17.95 \mathrm{~g} / \mathrm{cm}^{2}$ radius $), \mathrm{Al}\left(9.74 \mathrm{~g} / \mathrm{cm}^{2}\right.$ thick, $9.81 \mathrm{~g} / \mathrm{cm}^{2}$ radius $)$, and $\mathrm{Be}\left(11.67 \mathrm{~g} / \mathrm{cm}^{2}\right.$ thick, $6.72 \mathrm{~g} / \mathrm{cm}^{2}$ radius) cylinders. The targets are thick for electrons but not for photons. The targets were surrounded by a stainless steel target chamber, except for the $30^{\circ}, 60^{\circ}$, and $90^{\circ}$ mea- 
surements. There was an additional $\mathrm{Al}$ exit window downstream of the target. The photons then passed through a $\mathrm{Pb}$ collimator prior to being collected in a $\mathrm{NaI}$ detector.

2. Monte Carlo Simulation Description. The Monte Carlo simulation was designed to match the experiment as faithfully as possible. See Appendix B.1 and B.2 for sample MCNP input templates. The work of DeMarco, ${ }^{5}$ who performed a similar study using MCNP4A, was used extensively as a reference. The Al target exit window, side walls of the stainless steel target chamber, and $\mathrm{Pb}$ collimator were not included in the simulation since the published experimental results are corrected for these factors. The simulations were done in a vacuum, since the experimental data is also corrected for attenuation in air. The target dimensions corresponded exactly to the published values. The thicknesses for the Ti exit window and Si beam monitory system were taken from the published values, although the radial dimensions were estimated. Further uncertainty is introduced in modeling the stainless steel entrance window. In the MCNP model, the stainless steel was defined as $18 \%$ (weight fraction) chromium, $8 \%$ nickel, and $76 \%$ iron. This model was based on typical 304 stainless steel, neglecting the trace impurities.

The bremsstrahlung yields were tallied using cell flux and detector flux tallies. The spectral data over individual energy bins were tallied, although the primary item of interest was the bremsstrahlung yield integrated over all energies. The tallies were multiplied by the source-todetector distance (SDD) to convert the tally units from photons per $\mathrm{cm}^{2}$ to photons per steradian. The SDD of $300 \mathrm{~cm}$ is defined from the upstream surface of the target. The cell tallies were based on the union of two cones and two spheres, which forms an annular spherical region. An angular range of $0.5^{\circ}$ and a radial thickness of $1 \mathrm{~mm}$ were used. Ring detectors were defined according to the SDD and a ring radius which reproduces the desired angle. Since the geometry is cylindrically symmetric, ring detectors were used rather than point detectors for maximum efficiency. The simulation geometry is shown in Fig. 1 for some arbitrary angle $\theta$.

Detector and cell flux tallies are calculated in fundamentally different ways. The cell tally for flux is a track length estimate, in which the time integrated flux is estimated by the summing $W T_{l} / V$, where $W$ is the particle weight, $T_{l}$ is the track length of the particle in the cell, and $V$ is the cell volume. Conversely, a detector tally is a deterministic estimate of the flux at a point in space, or in the case of a ring detector tally, at a point sampled from some location on a ring. The detector flux is calculated ${ }^{1}$ 


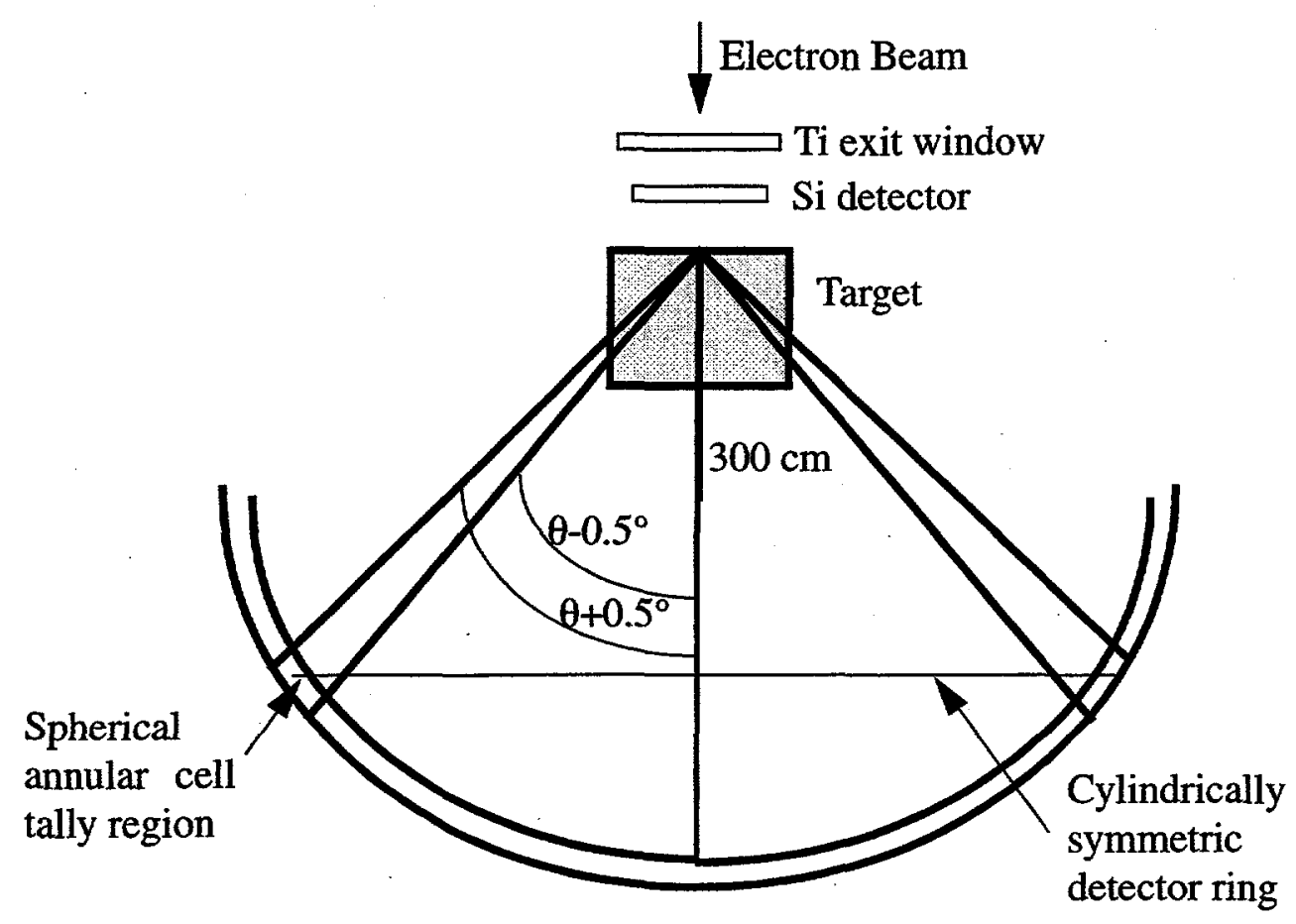

Fig. 1. MCNP Simulation Geometry.

$$
\Phi(\mathbf{r}, E, \mu)=\frac{W p(\mu) e^{-\lambda}}{2 \pi R^{2}}
$$

where $2 \mathrm{p}(\mu)$ is the probability density function at $\mu$, the cosine of the angle between the particle trajectory and the direction to the detector; $R$ is the distance from the source or collision event to the detector; and

$$
\lambda=\int_{0}^{R} \Sigma_{t}(s) d s
$$

which is the total number of mean free paths integrated over the trajectory from the source or collision event to the detector; $\Sigma_{t}$ is the total macroscopic cross section.

The exponential term of Eq. (3) accounts for the attenuation between the present event and the detector point, and a $1 / 4 \pi R^{2}$ term accounts for the solid angle effect. The $\mathrm{p}(\mu)$ term accounts for the probability of scattering toward the detector instead of the direction selected in the random 
walk. Each contribution to the detector can be thought of as transporting a "pseudoparticle" to the detector.

In addition to the integrated bremsstrahlung yield, the mean energy of the spectra for several angles was calculated. This was done either by taking a flux-weighted average over individual energy bins or by dividing a $* \mathrm{~F} 4$ (energy times flux) tally by the F4 tally (flux) over the total energy bin.

3. Results. The integrated bremsstrahlung yields for $\mathrm{Pb}, \mathrm{Al}$, and $\mathrm{Be}$ are presented in Figs. 2-4. These results were obtained using the default electron and photon settings in MCNP4B. Simulations for angles between $0^{\circ}$ and $10^{\circ}$ include the stainless steel entrance window, whereas angles greater than $10^{\circ}$ had no stainless steel window. These factors are consistent with the experiment.

Lead

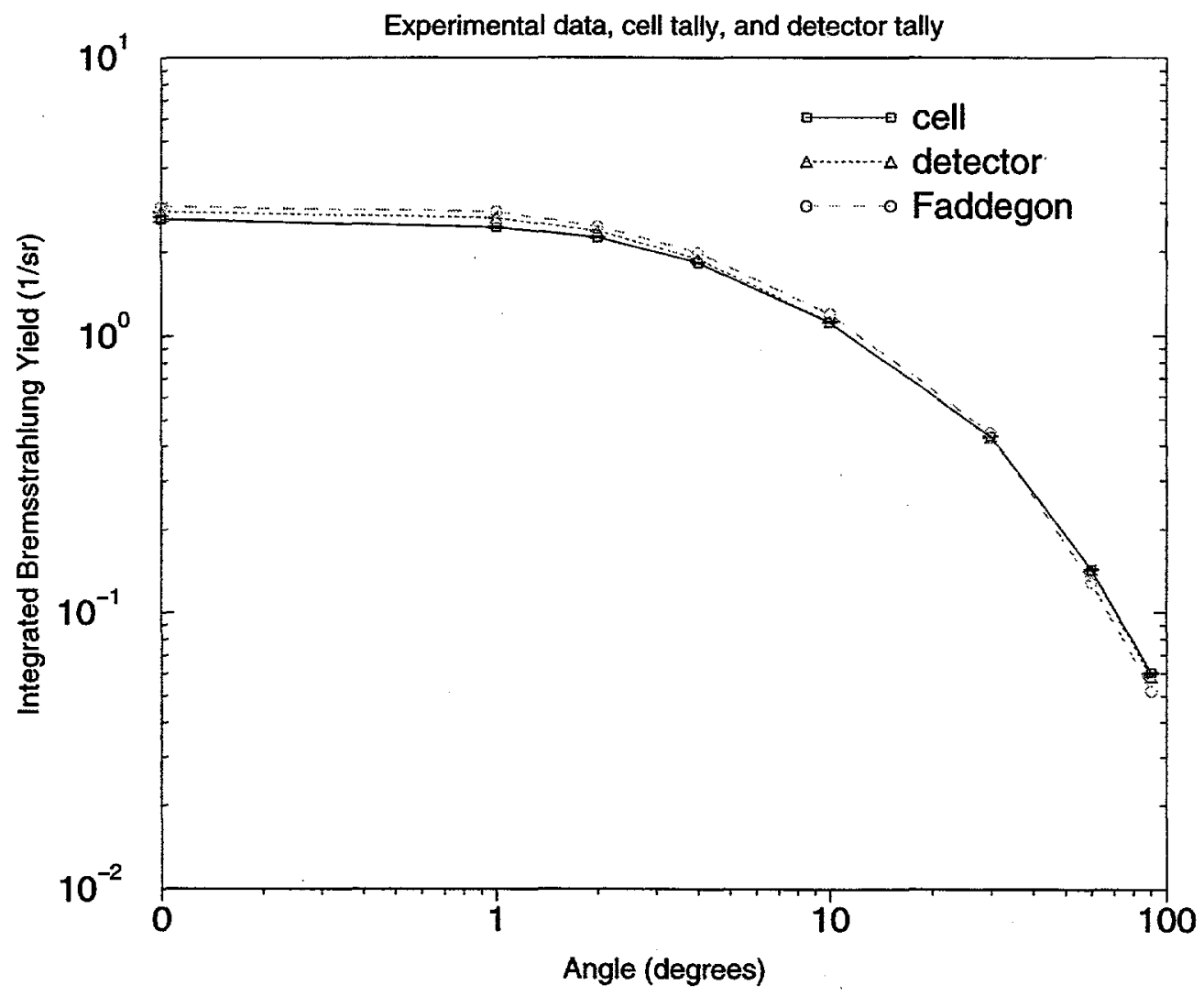

Fig. 2. Integrated Bremsstrahlung Yield vs Angle for $\mathbf{P b}$. 
Figure 2 shows that the discrepancies between experiment and simulation are greatest for the forward directed angles and for $90^{\circ}$. It is interesting to note that the detector tally seems to track the experiment better than the cell tally, even though more detailed physics is used for the cell tally for electron photon problems (see Sec. 4.1). The largest difference between experiment and simulation is $16 \%$ at $90^{\circ}$ for cell tallies, and $13 \%$ at $90^{\circ}$ for detector tallies. The error bars for the $0^{\circ}$ and $90^{\circ}$ simulations were generally the largest, because of the low intensity at $90^{\circ}$ and smaller for the solid angle at $0^{\circ}$. Table I gives the tabular data for Al. The numbers in parentheses are the percent errors.

Figure 3 shows the integrated bremsstrahlung yields as a function of angle for Al. The tabular data are given in Table II. The largest deviation for the MCNP simulation and experiment is $14 \%$ at $90^{\circ}$, for both cell and detector tallies. Figure 4 shows the integrated bremsstrahlung yields for Be. The tabular data are given in Table III. In this case, the $90^{\circ}$ data agree within statistical error for both cell and detector tallies, but the $0^{\circ}$ cell tally differs from experiment by about $15 \%$. For detector tallies for the materials studied, the calculated bremsstrahlung yields agree with experiment within statistics for a $68 \%$ confidence interval. For cell tallies for the materials studied, MCNP is usually able to predict the experimental integrated bremsstrahlung yields to within two standard deviations of the experimental and calculational uncertainties.

TABLE I: INTEGRATED BREMSSTRAHLUNG YIELD VS ANGLE FOR PB

\begin{tabular}{|c|c|c|c|}
\hline Angle & $\begin{array}{c}\text { 4B } \\
\text { Cell }\end{array}$ & $\begin{array}{c}\text { 4B } \\
\text { Detector }\end{array}$ & Faddegon \\
\hline \hline 0 & $2.640 \mathrm{E}+00(2.0)^{*}$ & $2.797 \mathrm{E}+00(0.3)$ & $2.92 \mathrm{E}+00(5.0)$ \\
1 & $2.463 \mathrm{E}+00(0.8)$ & $2.667 \mathrm{E}+00(0.3)$ & $2.80 \mathrm{E}+00(5.0)$ \\
2 & $2.255 \mathrm{E}+00(0.6)$ & $2.380 \mathrm{E}+00(0.3)$ & $2.48 \mathrm{E}+00(5.0)$ \\
4 & $1.835 \mathrm{E}+00(0.4)$ & $1.883 \mathrm{E}+00(0.3)$ & $1.99 \mathrm{E}+00(5.0)$ \\
10 & $1.122 \mathrm{E}+00(0.4)$ & $1.125 \mathrm{E}+00(0.3)$ & $1.2 \mathrm{E}+00(5.0)$ \\
30 & $4.338 \mathrm{E}-01(0.4)$ & $4.310 \mathrm{E}-01(0.5)$ & $4.47 \mathrm{E}-01(5.0)$ \\
60 & $1.444 \mathrm{E}-01(0.5)$ & $1.434 \mathrm{E}-01(0.5)$ & $1.29 \mathrm{E}-01(5.0)$ \\
90 & $6.029 \mathrm{E}-02(0.7)$ & $5.860 \mathrm{E}-02(0.7)$ & $5.19 \mathrm{E}-02(7.0)$ \\
\hline
\end{tabular}

${ }^{*}$ read as $2.640 \times 10^{0}$ with $2.0 \%$ error 


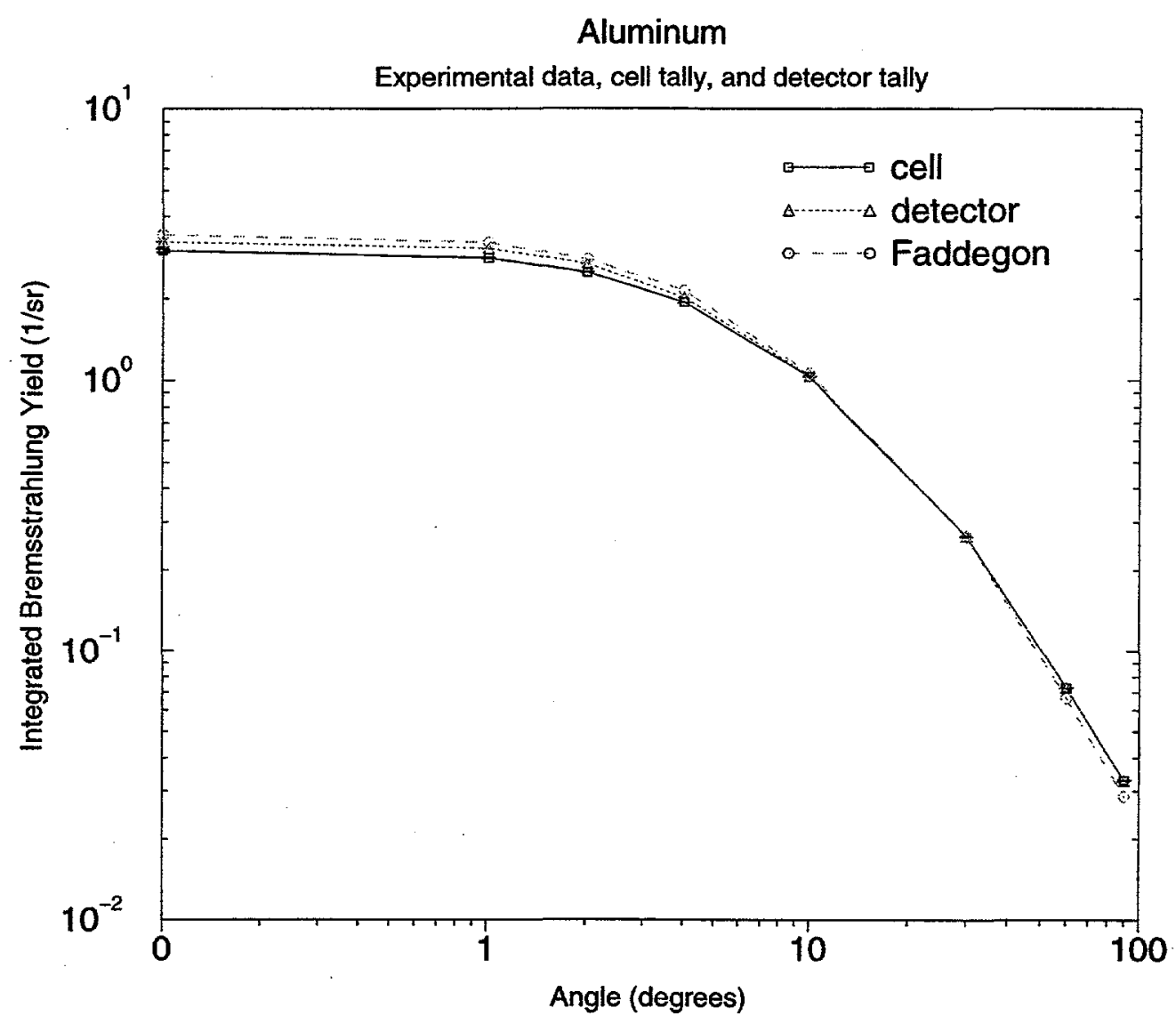

Fig. 3. Integrated Bremsstrahlung Yield vs Angle for Al.

TABLE II: INTEGRATED BREMSSTRAHLUNG YIELD VS ANGLE FOR AL

\begin{tabular}{|c|c|c|c|}
\hline Angle & $\begin{array}{c}\text { 4B } \\
\text { Cell }\end{array}$ & $\begin{array}{c}\text { 4B } \\
\text { Detector }\end{array}$ & Faddegon \\
\hline \hline 0 & $2.991 \mathrm{E}+00(1.5)^{*}$ & $3.224 \mathrm{E}+00(0.2)$ & $3.42 \mathrm{E}+00(5.0)$ \\
1 & $2.802 \mathrm{E}+00(0.6)$ & $3.045 \mathrm{E}+00(0.2)$ & $3.21 \mathrm{E}+00(5.0)$ \\
2 & $2.494 \mathrm{E}+00(0.4)$ & $2.670 \mathrm{E}+00(0.2)$ & $2.78 \mathrm{E}+00(5.0)$ \\
4 & $1.931 \mathrm{E}+00(0.3)$ & $2.009 \mathrm{E}+00(0.2)$ & $2.14 \mathrm{E}+00(5.0)$ \\
10 & $1.033 \mathrm{E}+00(0.3)$ & $1.043 \mathrm{E}+00(0.2)$ & $1.06 \mathrm{E}+00(5.0)$ \\
30 & $2.664 \mathrm{E}-01(0.3)$ & $2.673 \mathrm{E}-01(0.3)$ & $2.65 \mathrm{E}-01(5.0)$ \\
60 & $7.284 \mathrm{E}-02(0.5)$ & $7.278 \mathrm{E}-02(0.3)$ & $6.66 \mathrm{E}-02(6.0)$ \\
90 & $3.294 \mathrm{E}-02(0.7)$ & $3.280 \mathrm{E}-02(0.3)$ & $2.87 \mathrm{E}-02(6.0)$ \\
\hline
\end{tabular}

$*_{\text {read as }} 2.991 \times 10^{0}$ with $1.5 \%$ error 


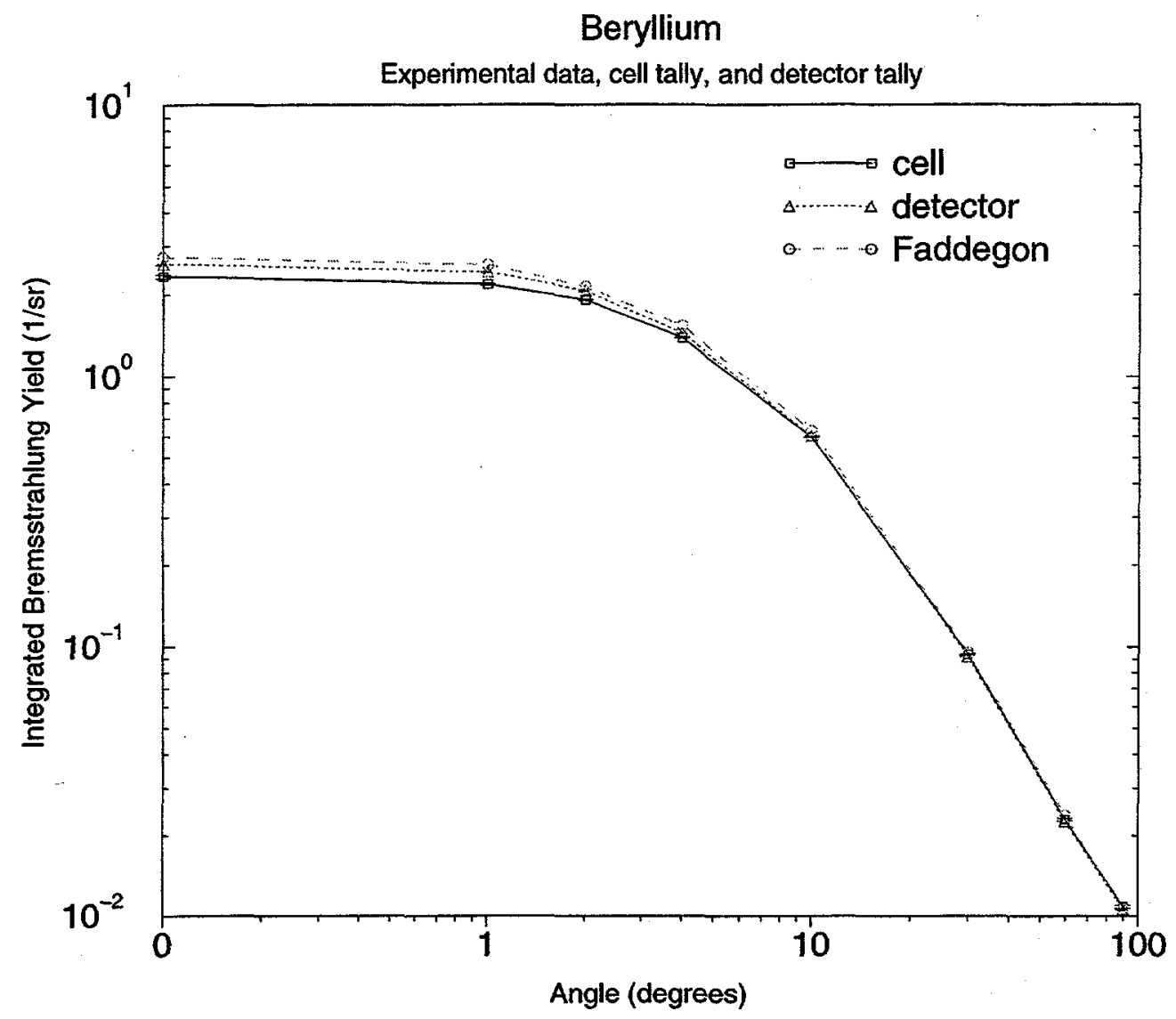

Fig. 4. Integrated Bremsstrahlung Yield vs Angle for Be.

TABLE III: INTEGRATED BREMSSTRAHLUNG YIELD VS ANGLE FOR BE

\begin{tabular}{|c|c|c|c|}
\hline Angle & $\begin{array}{c}\text { 4B } \\
\text { Cell }\end{array}$ & $\begin{array}{c}\text { 4B } \\
\text { Detector }\end{array}$ & Faddegon \\
\hline \hline 0 & $2.332 \mathrm{E}+00(1.8)^{*}$ & $2.588 \mathrm{E}+00(0.2)$ & $2.73 \mathrm{E}+00(5.1)$ \\
1 & $2.176 \mathrm{E}+00(0.7)$ & $2.412 \mathrm{E}+00(0.2)$ & $2.57 \mathrm{E}+00(5.1)$ \\
2 & $1.917 \mathrm{E}+00(0.5)$ & $2.058 \mathrm{E}+00(0.2)$ & $2.14 \mathrm{E}+00(5.1)$ \\
4 & $1.390 \mathrm{E}+00(0.4)$ & $1.444 \mathrm{E}+00(0.2)$ & $1.54 \mathrm{E}+00(5.0)$ \\
10 & $5.977 \mathrm{E}-01(0.4)$ & $6.030 \mathrm{E}-01(0.3)$ & $6.30 \mathrm{E}-01(5.1)$ \\
30 & $9.368 \mathrm{E}-02(0.6)$ & $9.195 \mathrm{E}-02(0.3)$ & $9.49 \mathrm{E}-02(5.1)$ \\
60 & $2.294 \mathrm{E}-02(0.9)$ & $2.265 \mathrm{E}-02(0.3)$ & $2.38 \mathrm{E}-02(5.9)$ \\
90 & $1.079 \mathrm{E}-02(1.2)$ & $1.053 \mathrm{E}-02(0.3)$ & $1.06 \mathrm{E}-02(7.0)$ \\
\hline
\end{tabular}

*Tead as $2.332 \times 10^{0}$ with $1.8 \%$ error 
Figures 5-7 compare experimental and simulation results for the bremsstrahlung energy spectra for lead, aluminum, and beryllium. These plots compare the default MCNP cell tally with experimental data for $10^{\circ}$. The simulations show excellent agreement for each material, although MCNP deviates from experiment at very low photon energies for lead. These results show that MCNP can accurately calculate both the integrated bremsstrahlung yields as well as the detailed photon energy spectra.

The mean energy of the bremsstrahlung spectrum for each target was also calculated and compared with the experimental data for a few emission angles. Table IV summarizes the results of these simulations. The numbers in parentheses are the percent errors. The $\mathrm{Al}$ and $\mathrm{Pb}$ results agreed quite well with experiment, with only the $0^{\circ}$ data having statistically significant deviations. The Be simulation results did not track the experiment as well as the other materials did; the $0^{\circ}, 60^{\circ}, 90^{\circ}$ tallies differed by a few percent outside the $1 \sigma$ error bars. This level of agreement is sufficient to form a $68 \%$ confidence interval and shows that the calculated mean energies agree with the experiment within statistical uncertainty.

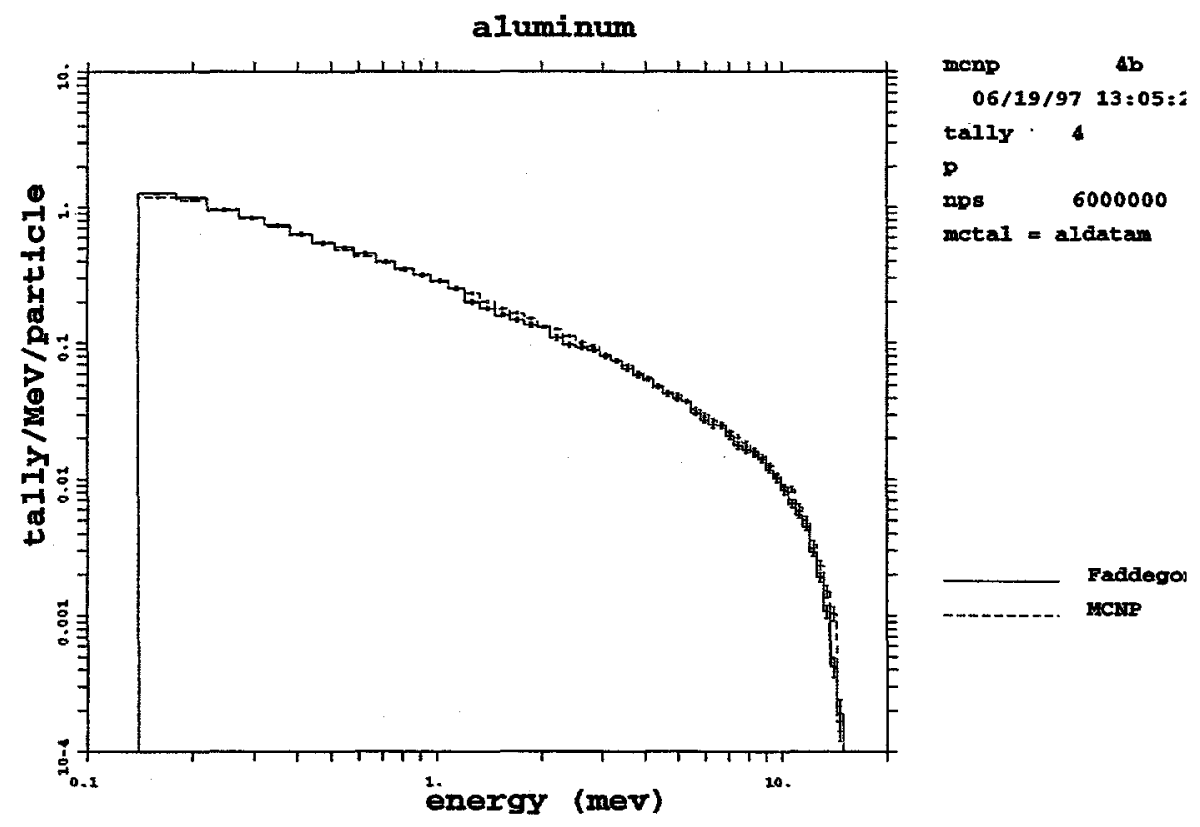

Fig. 5. Comparison of experimental and MCNP cell tally bremsstrahlung spectra for aluminum at $\mathbf{1 0}^{\circ}$. 


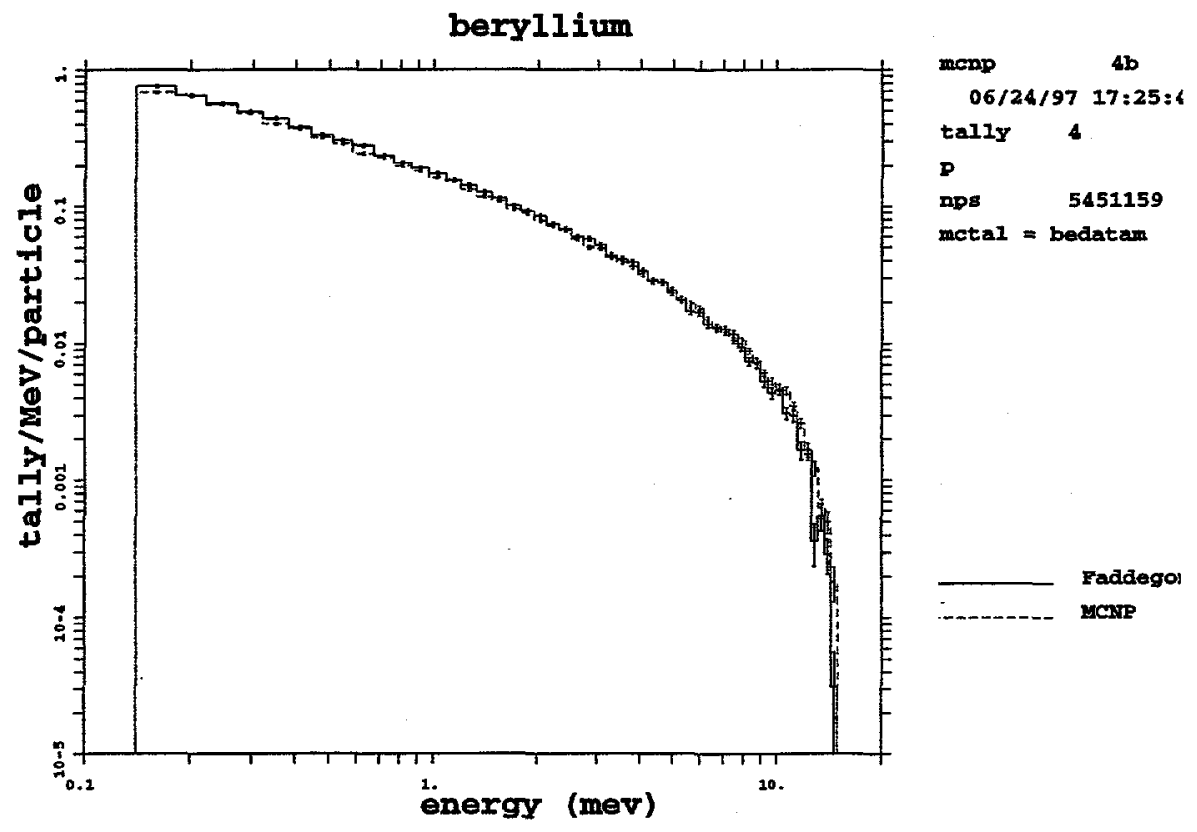

Fig. 6. Comparison of experimental and MCNP cell tally bremsstrahlung spectra for beryllium at $10^{\circ}$.

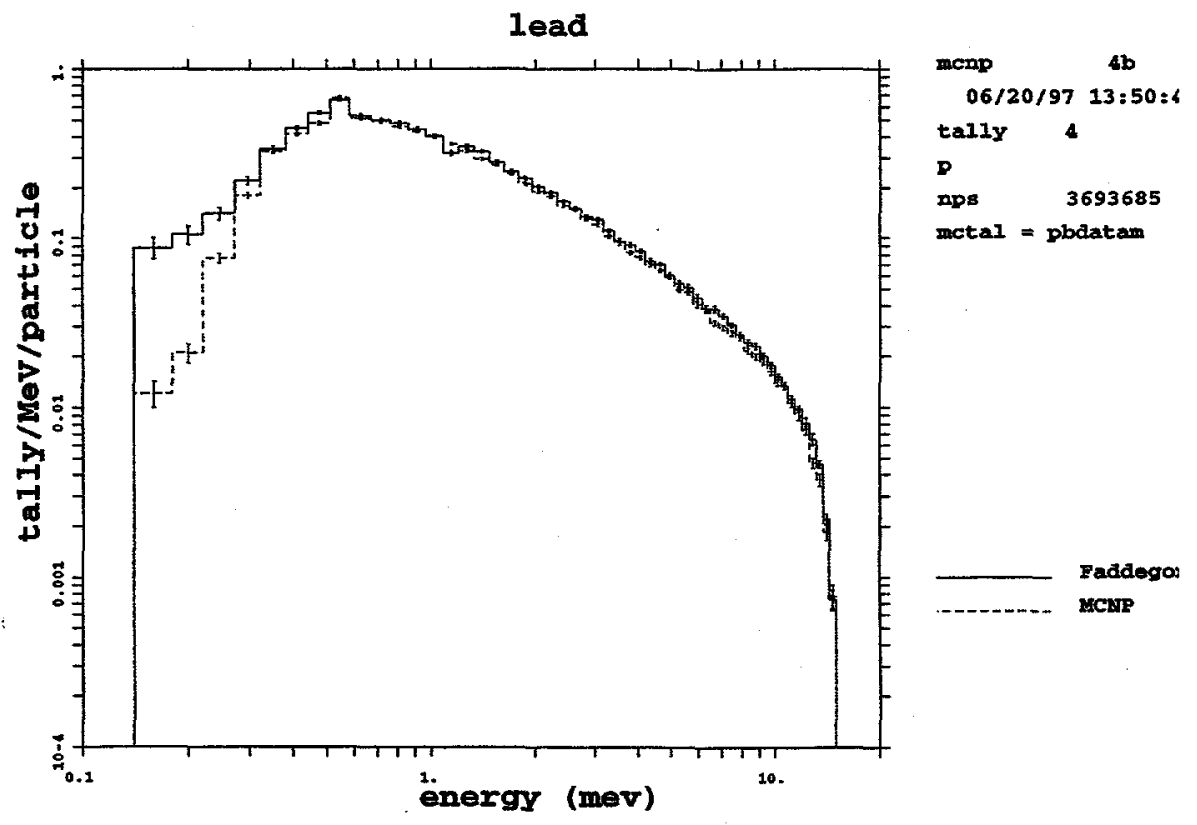

Fig. 7. Comparison of experimental and MCNP cell tally bremsstrahlung spectra for lead at $10^{\circ}$. 
TABLE IV: BREMSSTRAHLUNG SPECTRA MEAN ENERGIES

\begin{tabular}{|c|c|c|c|c|}
\hline \multirow{2}{*}{ Target } & Angle & \multicolumn{3}{|c|}{ Mean Energy (MeV) } \\
\hline & & 4B cell & 4B detector & Faddegon \\
\hline \hline $\mathrm{Be}$ & 0 & $2.62(3.2)$ & $2.68(0.5)$ & $2.86(4.1)$ \\
& 10 & $2.08(0.7)$ & $2.04(0.6)$ & $2.09(3.4)$ \\
& 30 & $1.14(1.1)$ & $1.14(0.9)$ & $1.15(2.4)$ \\
& 60 & $0.55(1.7)$ & $0.55(0.9)$ & $0.596(3.0)$ \\
& 90 & $0.36(2.3)$ & $0.348(0.9)$ & $0.379(2.5)$ \\
\hline $\mathrm{Al}$ & 0 & $2.61(10.5)$ & $2.59(1.5)$ & $2.74(3.7)$ \\
& 10 & $2.11(1.9)$ & $2.09(1.6)$ & $2.17(3.1)$ \\
& 30 & $1.39(2.0)$ & $1.38(1.8)$ & $1.42(2.9)$ \\
& 60 & $0.84(2.7)$ & $0.83(1.7)$ & $0.83(3.0)$ \\
& 90 & $0.55(3.2)$ & $0.56(1.6)$ & $0.57(2.5)$ \\
\hline $\mathrm{Pb}$ & 0 & $3.01(2.8)$ & $3.08(0.5)$ & $3.22(3.5)$ \\
& 10 & $2.72(0.7)$ & $2.73(0.6)$ & $2.77(3.0)$ \\
& 30 & $2.216(0.5)$ & $2.21(1.0)$ & $2.25(3.0)$ \\
& 60 & $1.827(0.6)$ & $1.84(1.1)$ & $1.81(2.8)$ \\
& 90 & $1.800(0.8)$ & $1.84(1.4)$ & $1.84(2.6)$ \\
\hline
\end{tabular}

\section{B. O'Dell et al., Experiment}

1. Experimental Methods. O'Dell et al., ${ }^{8}$ measured the thick-target bremsstrahlung spectra for 5.3 to $20.9 \mathrm{MeV}$ electrons incident on a gold-tungsten target. The bremsstrahlung target was $0.49 \mathrm{~g} / \mathrm{cm}^{2}$ of tungsten followed by $0.245 \mathrm{~g} / \mathrm{cm}^{2}$ of gold. The spectra were measured using a technique based on deuteron photodisintegration. This method is limited to measuring photon energies above $3 \mathrm{MeV}^{6}$ The electrons were incident on the bremsstrahlung target, and the resulting photons interact with a secondary $\mathrm{D}_{2} \mathrm{O}$ target, which provides a source of photoneutrons. The neutrons produced above the $D(\gamma, n)$ p reaction threshold of $2.23 \mathrm{MeV}$ were analyzed using time-of-flight techniques. This analysis gives absolute bremsstrahlung spectra in units of photons per $\mathrm{MeV}$ per steradian per incident electron. Figure 8 illustrates the experimental setup.

2. Monte Carlo Simulation Description. The bremsstrahlung target was modeled as a thin cylinder of tungsten followed by a thin cylinder of gold. Simulations using 10.0 and 20.9 $\mathrm{MeV}$ incident electrons were done. The secondary target was modeled with dimensions of $1.25 \mathrm{x}$ 0.25 and $0.5 \times 0.25$ in. for the 10.0 and 20.9 measurements, respectively. The photon spectrum was tallied over the entire face of the cell. This tally is important since the bremsstrahlung yield is fairly sensitive to angle, especially near $0^{\circ} .^{10}$ Electron and photon low-energy cutoffs were set to 4.0 MeV, which mirror the published experimental result. As shown in Fig. 8, only the electron 
beam and bremsstrahlung target were modeled, with the photons being tallied over the face of the $\mathrm{D}_{2} \mathrm{O}$ target. See Appendix B.3 for the input template.

3. Results. Calculated and experimental bremsstrahlung spectra are shown in Figs. 9 and 10 for incident electron energies of 10.0 and $20.9 \mathrm{MeV}$, respectively. Error bars for the experimental data are based on O'Dell's estimate that the errors range from 5 to $10 \%$, except at higher photon energies where poor counting statistics further increase the experimental error. There is good overall agreement between calculation and experiment at both energies. All of the points agree within experimental error, with the exception of the very first data point for the incident energy of $10.0 \mathrm{MeV}$. There also appear to be some minor discontinuities in the MCNP simulations. These are most likely statistical in nature and are not a reflection of the cross-section data. This fact can be verified by running the simulation for more histories.

Table $\mathrm{V}$ gives the integrated bremsstrahlung yields for the $\mathrm{Au} / \mathrm{W}$ target at 10.0 and 20.9 MeV. The results for O'Dell were obtained by integrating the published spectra, whereas the MCNP result was automatically obtained from the cell tally. The results show that MCNP agrees with experiment to within $5 \%$.

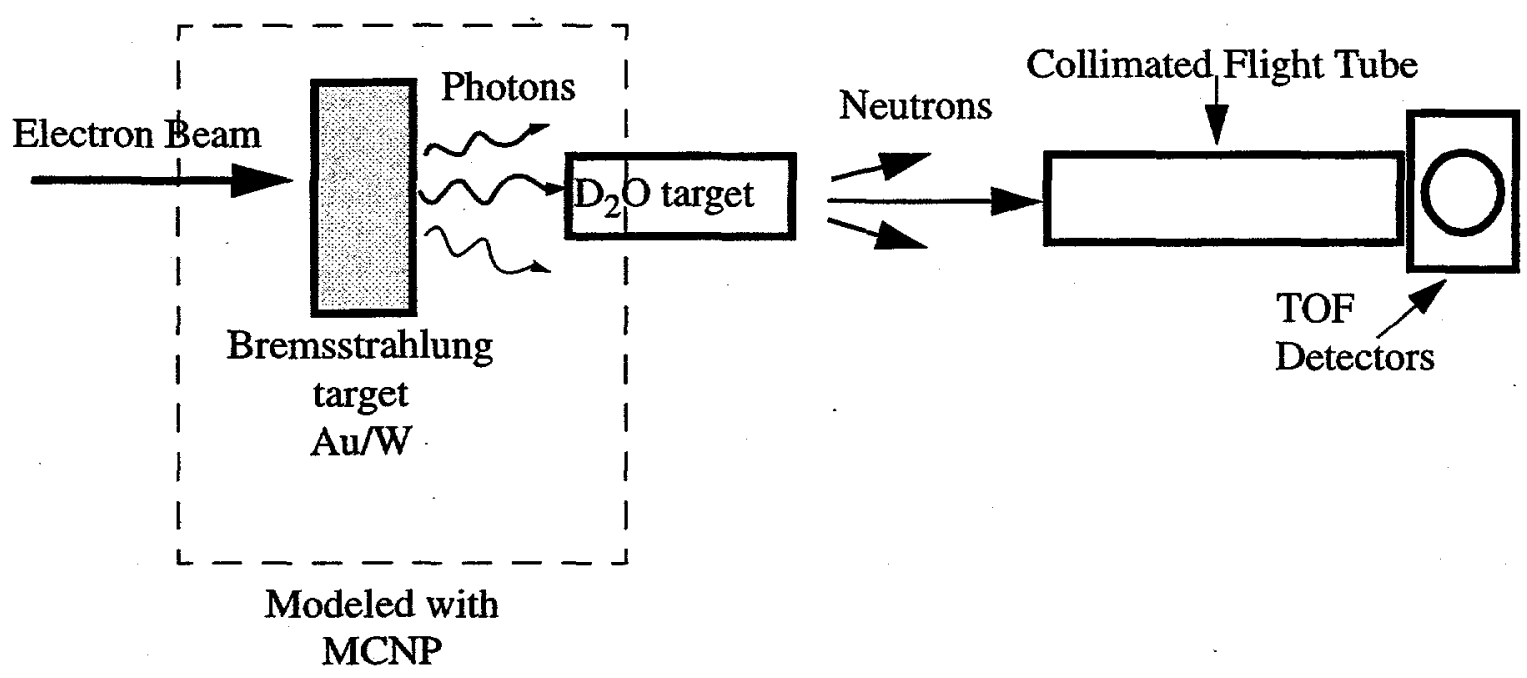

Fig. 8. Experimental setup for O'Dell et al. 


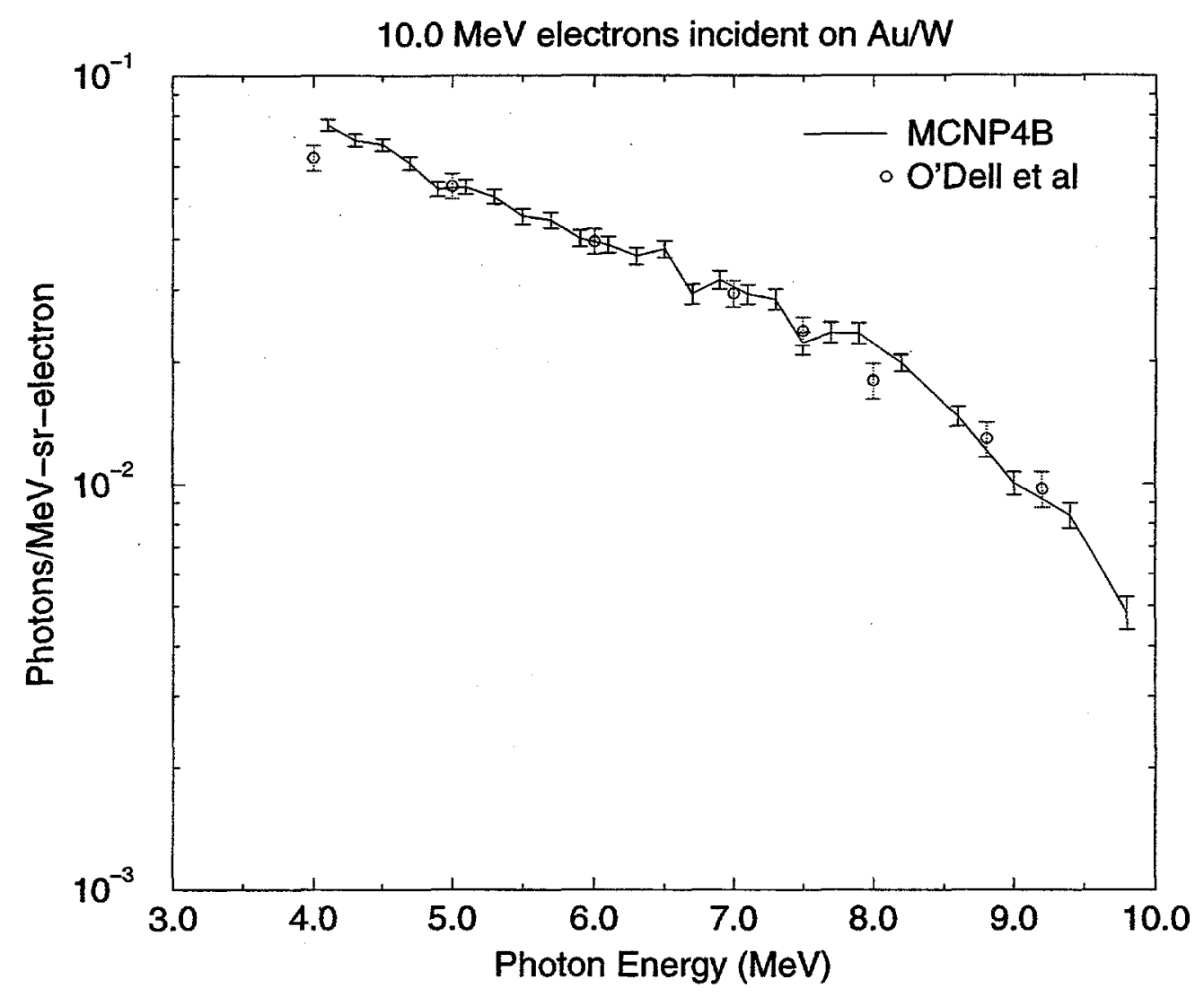

Fig. 9. Bremsstrahlung Energy Spectrum, 10.0 MeV electrons incident on Au/W.

\section{Starfelt and Koch Experiment}

1. Experimental Methods. Starfelt and Koch ${ }^{9}$ have also measured thick target bremsstrahlung spectra. They report photon spectra for $9.66 \mathrm{MeV}$ electrons incident on a tungsten target for $0^{\circ}$ and $12^{\circ}$. They used an electron beam from a $50 \mathrm{MeV}$ betatron which passed through a system of Lucite collimators in order to minimize the angular spread of the beam. The electron current in the target was not measured so the spectra are not absolute. The beam was focused on the bremsstrahlung target by an iron-core magnetic lens. Targets were mounted on aluminum rings $1 \mathrm{~mm}$ thick with $52 \mathrm{~mm}$ inside diameters. The tungsten target was $5.8 \mathrm{~g} / \mathrm{cm}^{2}$ thick. The bremsstrahlung photons pass through an aluminum window, cadmium filter, and lead collimator before impinging on a $\mathrm{NaI}(\mathrm{Tl})$ spectrometer. Figure 11 illustrates the experimental setup. 


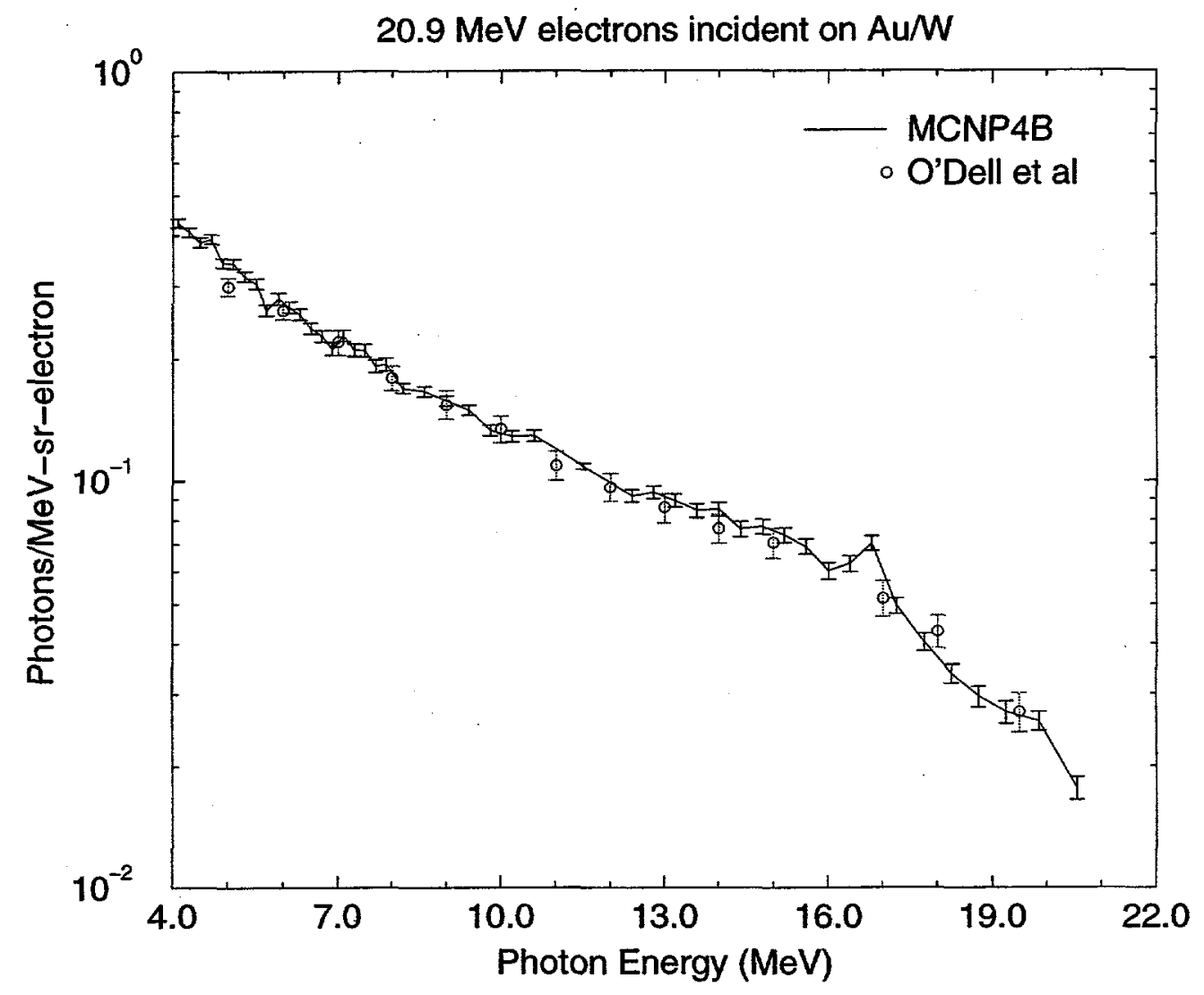

Fig. 10. Bremsstrahlung Energy Spectrum, $20.9 \mathrm{MeV}$ electrons incident on Au/W.

TABLE V: INTEGRATED YIELDS FOR AU/W FOR O'DELL

\begin{tabular}{|c|c|c|}
\hline $\begin{array}{c}\text { Energy } \\
\text { (MeV) }\end{array}$ & O'Dell & MCNP \\
\hline \hline 10.0 & $0.1826\left(23.6,7.5^{*}\right)$ & $0.1949(0.93)$ \\
20.9 & $2.0929(29.0,7.5)$ & $2.1956(0.48)$ \\
\hline
\end{tabular}

*first number is propagated percent error, second is percent error in each experimental data point 


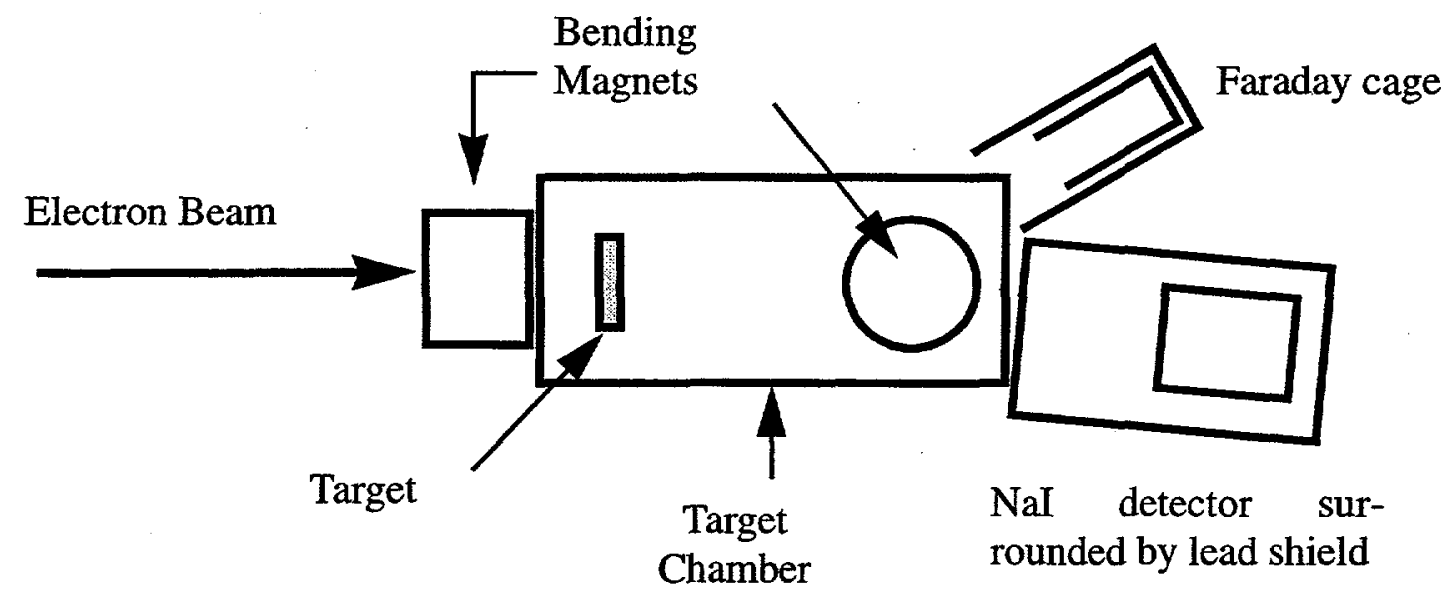

Fig. 11. Experimental setup for Starfelt and Koch.

2. Monte Carlo Simulation Description. Since the photon spectra were corrected for collimator effects and photon absorption between target and spectrometer, the only material included in the simulation was the target (see Appendix B.4). Electron and photon cutoff energies were set to $0.4 \mathrm{MeV}$. Cell and detector tallies were positioned at $0^{\circ}$ and $12^{\circ}$ using the same method as described in Section II.A.2. The cell tallies had an angular range of $0.7^{\circ}$, which is consistent with the experimental setup. To reconcile the differences between the relative experimental results and the absolute MCNP calculations, the experimental data were normalized to the simulation at the first (lowest) energy bin.

3. Results. Figures 12 and 13 give the $0^{\circ}$ and $12^{\circ}$ bremsstrahlung spectra as a function of photon energy, respectively. The MCNP results are cell tallies. The experimental and MCNP spectral shapes show good agreement for both angles and, in fact, agree within statistical uncertainty. The spectra agree particularly well for lower photon energies, which correspond to the highest photon yields. Error bars are not shown for the experimental data, but Starfelt and Koch estimate the uncertainties to range from about $3-4 \%$ at $1 \mathrm{MeV}$ to $11-17 \%$ at $9 \mathrm{MeV}$. The experimental errors become quite large for photon energies above $95 \%$ of the incident electron energy. Table VI shows a cell and detector comparison for integrated yield. These results are not compared to experiment because the experiment was not an absolute measurement. These results are consistent with the Faddegon comparisons in that the cell tallies are slightly lower than the detector tallies for forward angles. The values in parentheses are percent errors. 
0 degree

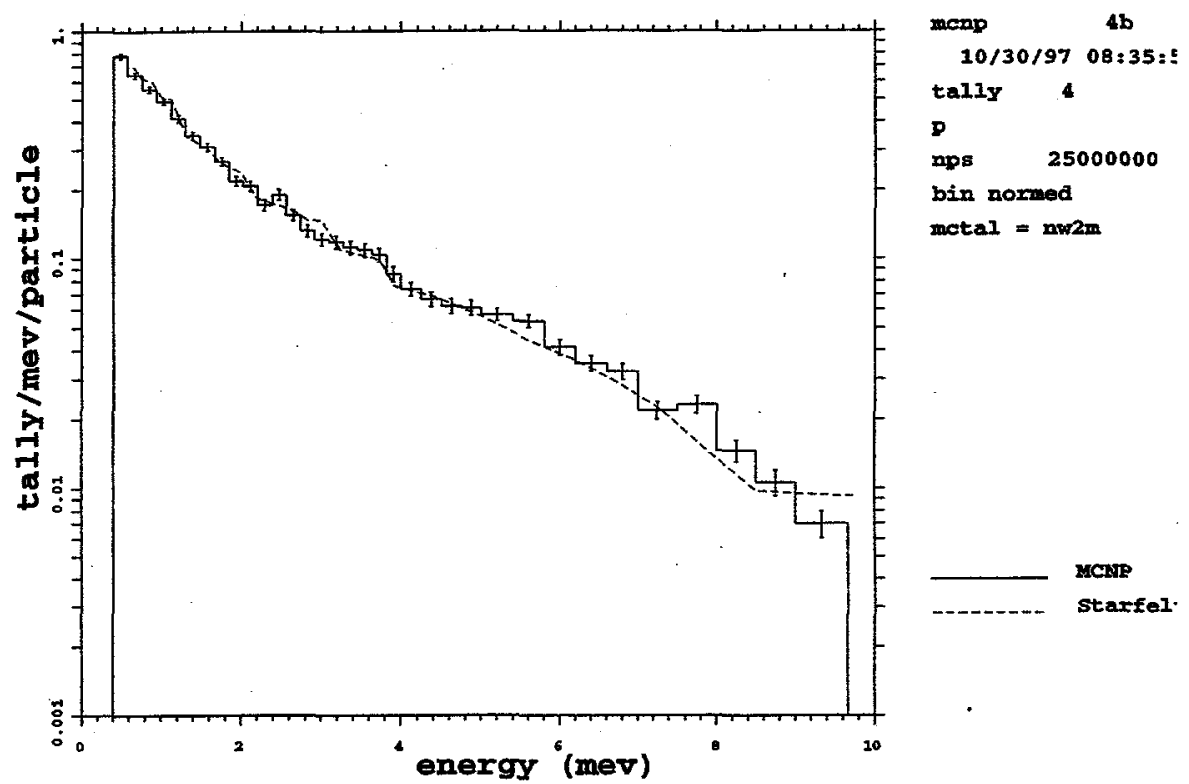

Fig. 12. Experimental and calculated bremsstrahlung spectrum at $0^{\circ}$ for $9.66 \mathrm{MeV}$ electrons incident on tungsten.

12 degrees

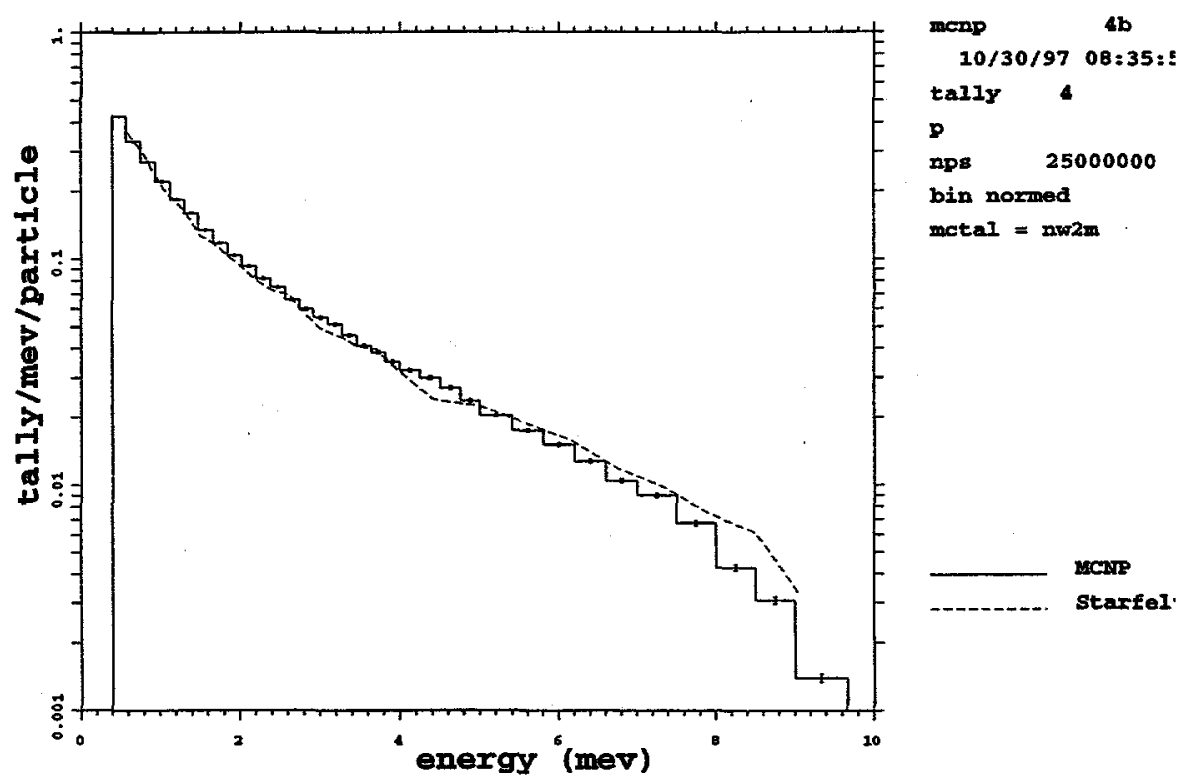

Fig. 13. Experimental and calculated bremsstrahlung spectrum at $12^{\circ}$ for $9.66 \mathrm{MeV}$ electrons incident on tungsten. 
TABLE VI: INTEGRATED YIELDS FOR TUNGSTEN FOR STARFELT AND KOCH

\begin{tabular}{|c|c|c|}
\hline Angle & Cell & Detector \\
\hline \hline 0 & $1.189(0.9)$ & $1.2615(0.1)$ \\
12 & $0.538(0.1)$ & $0.5417(0.2)$ \\
\hline
\end{tabular}

\section{ELECTRON TRANSMISSION AND BACKSCATTER}

This section of the report describes a series of electron transmission and backscatter comparisons. These calculations provide insight into the fundamental properties of Monte Carlo electron transport.

\section{A. Ebert et al., Experiment}

1. Experimental Methods. Ebert et al., ${ }^{11}$ give a tremendous amount of data for 4.0$12.0 \mathrm{MeV}$ monoenergetic electrons incident on a variety of solid targets. In this study, a few of the experimental transmission and backscatter experiments have been simulated using MCNP4B.

A beam of electrons of current $I_{0}$ incident on a planar target is backscattered, absorbed, and transmitted. During some time $\tau$, a charge $Q_{0}=I_{0} \tau$ is incident on the target. The transmission coefficient $\mathrm{T}$ is given by

$$
T=Q_{T} / Q_{0}=\left(Q_{T} /\left(Q_{B}+Q_{A}+Q_{T}\right)\right)
$$

where $Q_{T}$ is the charge transmitted through the target, $Q_{\mathrm{A}}$ is the charge absorbed in the target, and $Q_{B}$ is the charge backscattered from the target. The backscatter coefficient B is given by

$$
B=Q_{B} / Q_{0}=Q_{B} /\left(Q_{B}+Q_{A}+Q_{T}\right)
$$

Increasing the target thickness will only increase the backscatter coefficient to a certain limiting value. This effect is called the saturation backscatter coefficient.

In this experiment, the electron beam, generated by a linac, had an energy spread of about 1\%. The beam passed through two copper collimators, chosen as a compromise between a low $-\mathrm{Z}$, low-density material which would produce a large low-energy secondary electron background, and a high $\mathrm{Z}$, high-density material which would produce a high bremsstrahlung background. The maximum beam diameter allowed by the collimators was $0.6 \mathrm{~cm}$ at the target. 
The target chamber contained x-ray shielding, an insulated target holder, two large Faraday cups, and a carbon beam stop in addition to the collimator assembly. The Faraday cups were used to collect the transmitted and backscattered electrons. Bias rings, set to $-500 \mathrm{~V}$, were mounted in the Faraday cups to minimize the very low energy secondary electron current. The targets ranged in thickness from about 0.03 to $6.0 \mathrm{~g} / \mathrm{cm}^{2}$. The targets were either 2.0 or $8.0 \mathrm{~cm}$ in diameter. The larger targets ranged in linear thickness from 0.6 to $3.2 \mathrm{~cm}$. The target dimensions were chosen such that the target radius was greater than the sum of the beam radius and the maximum electron range. This configuration will minimize electron escape through the target sides, and approximate a semi-infinite slab geometry. The experimental geometry is described in Fig. 14.

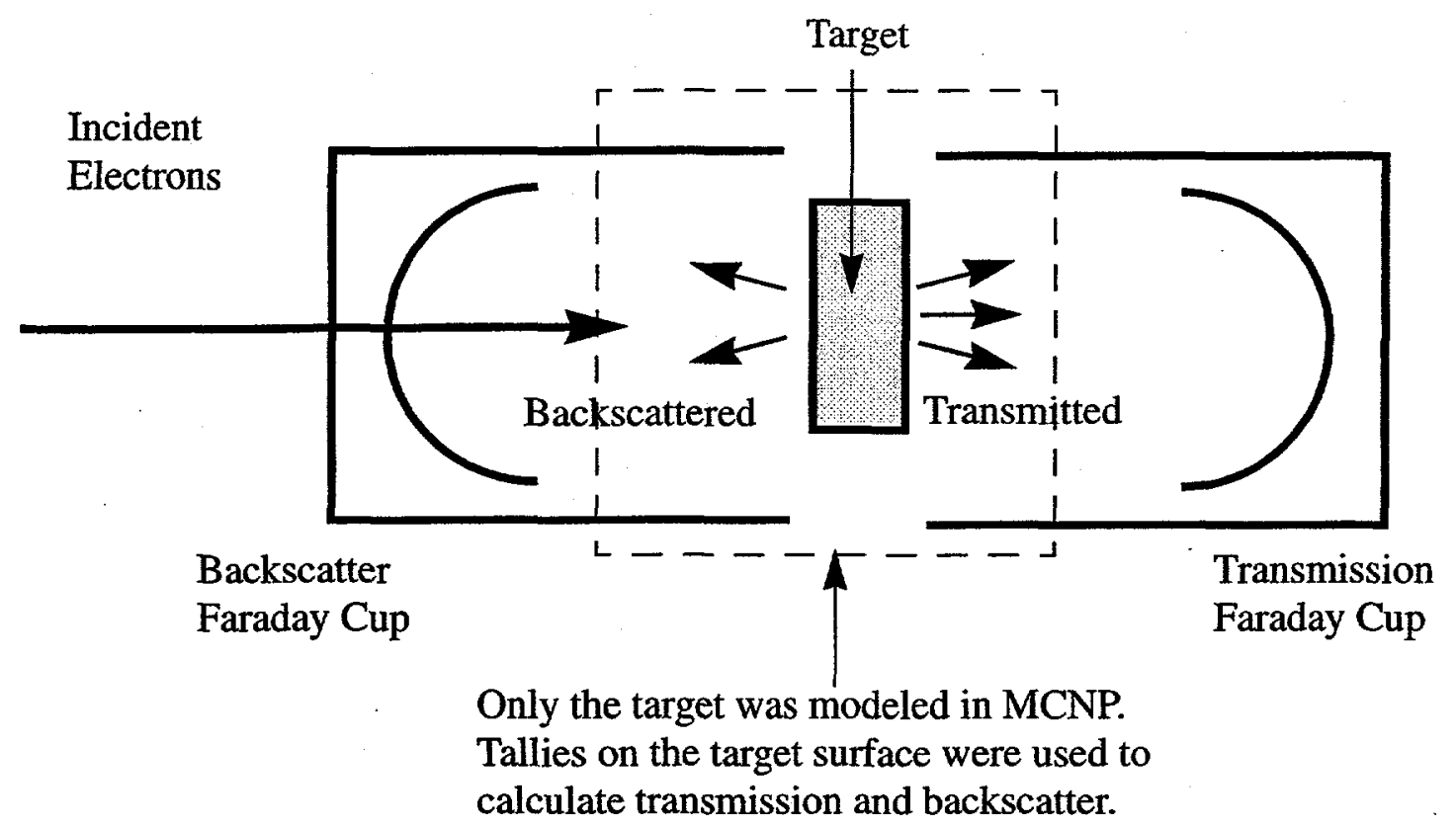

Fig. 14. Geometry for Ebert transmission and backscatter experiments.

2. Monte Carlo Simulation Description. The experimental geometry was greatly simplified for the MCNP simulations (see Appendix B.5). Only the target was explicitly modeled. The transmission and backscatter coefficients were calculated using current tallies at the target faces. This technique is much simpler than modeling the Faraday cup geometry, and ensures that there are no tally losses from solid angle effects. The current tallies were divided into two $\cos \theta$ bins, where $\theta$ is defined relative to the positive surface normal. The transmission 
coefficient was calculated by using a $\theta$ range of $0^{\circ}$ to $90^{\circ}$, whereas the backscatter coefficient used a $\theta$ range of $90^{\circ}$ to $180^{\circ}$. The simulations were done in coupled electron/photon mode and used the default cutoffs of $1 \mathrm{keV}$.

3. Results. Transmission coefficients for $10.2 \mathrm{MeV}$ electrons incident on $\mathrm{C}, \mathrm{Ag}$, and $\mathrm{U}$ foils of varying thicknesses are presented in Figs. 15-17. The experimental results and simulation results using MCNP4B default settings are shown. The simulations agree with experiment with varying degrees of success. The experimental uncertainties are estimated at $2 \%$; Ebert et al., only give a errors for transmission coefficients between 0.3 and 0.8 .

The silver simulations agree with experiment to the greatest degree. The maximum deviation between the default simulation and experiment is $17 \%$, whereas most of the transmission coefficients agree within $10 \%$. For the range of transmission coefficients for which experimental uncertainties are published, the simulation and experiment agree within statistics. For carbon, the default MCNP simulations disagree with experiment by as much as $90 \%$ for the last few data points, although the transmission coefficients for thicknesses less than $3.5 \mathrm{~g} / \mathrm{cm}^{2}$ differ within $10 \%$. The experimental uncertainties in the transmission coefficients for very thick targets may be quite large, which may help account for these discrepancies. For uranium, default MCNP agrees with experiment within 5-15\%.

The next three figures are presented in order of increasing $Z$. For carbon, the experimental data are lower than the simulation results. As $Z$ increases, the magnitude of the experimental data increases relative to the simulation data. These data indicate that some trend with $\mathrm{Z}$ is present in either the experiment or the Monte Carlo simulation.

Table VII shows the results of the backscatter benchmark calculations. The values in parentheses for the Monte Carlo simulations are the percent errors. The calculations were done for carbon, silver, and uranium foils at incident electron energies of 6.0, 8.0, and 10.2 MeV. Data from Dressel ${ }^{12}$ and Tabata ${ }^{16}$ are also included to show the wide range of experimental values that are in the literature. The default MCNP simulations agree with the Ebert experiment to within 8$20 \%$, with the exception of the values for carbon at $10.2 \mathrm{MeV}$, which disagree by $46 \%$. The errors for the experimental measurements are about 7-10\%. Disregarding this outlying value, the overall agreement is fair, and within $2 \sigma$. 


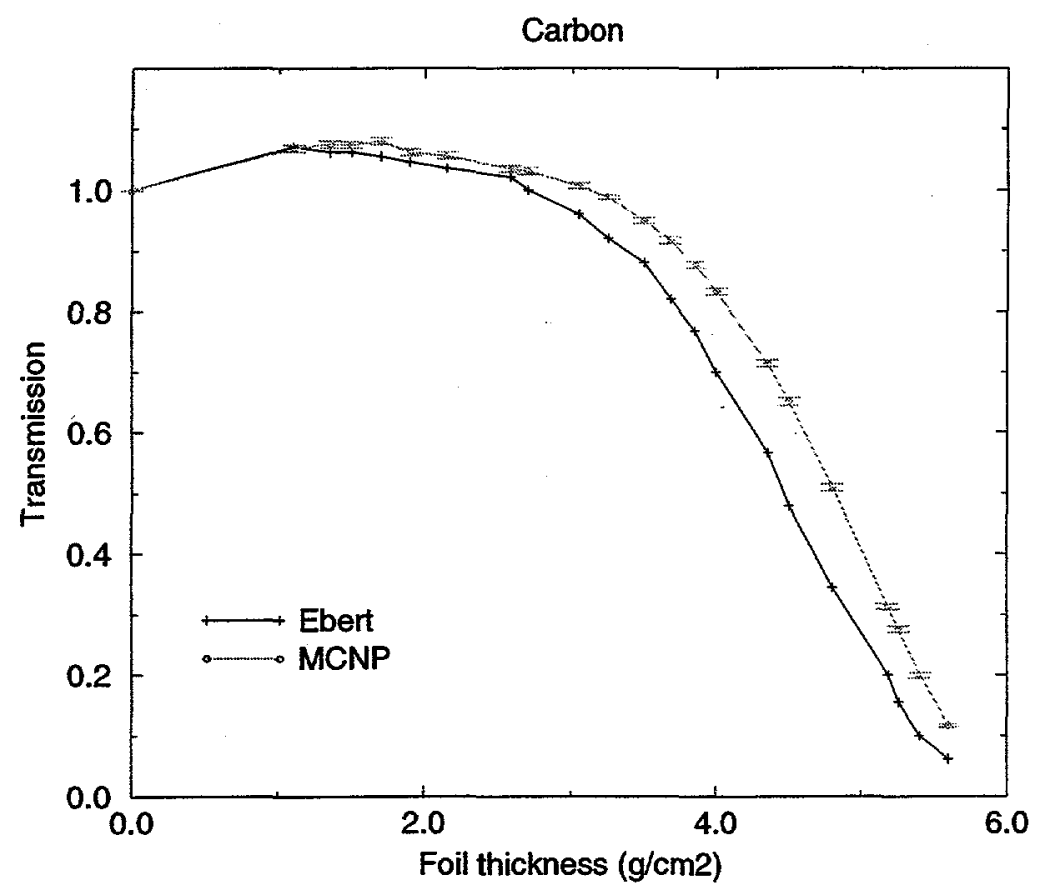

Fig. 15. Comparison of transmission coefficients for $10.2 \mathrm{MeV}$ electrons incident on $\mathrm{C}$ foils.

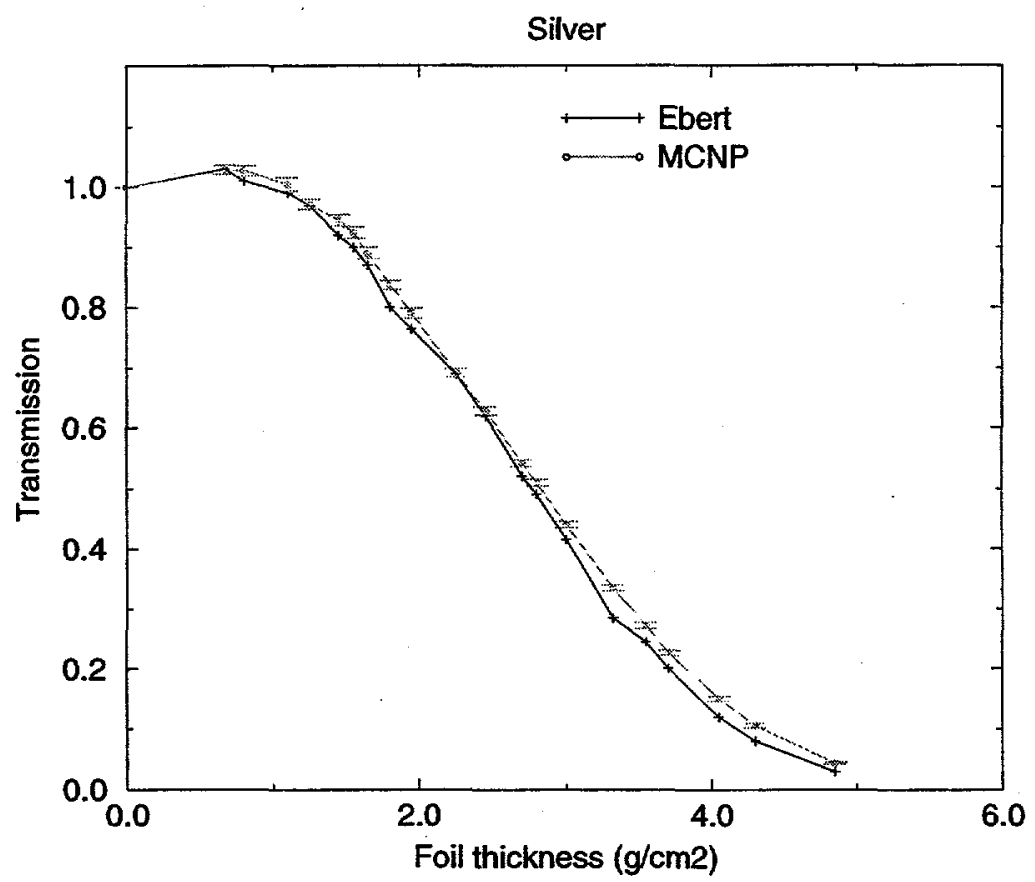

Fig. 16. Comparison of transmission coefficients for $10.2 \mathrm{MeV}$ electrons incident on $\mathrm{Ag}$ foils. 


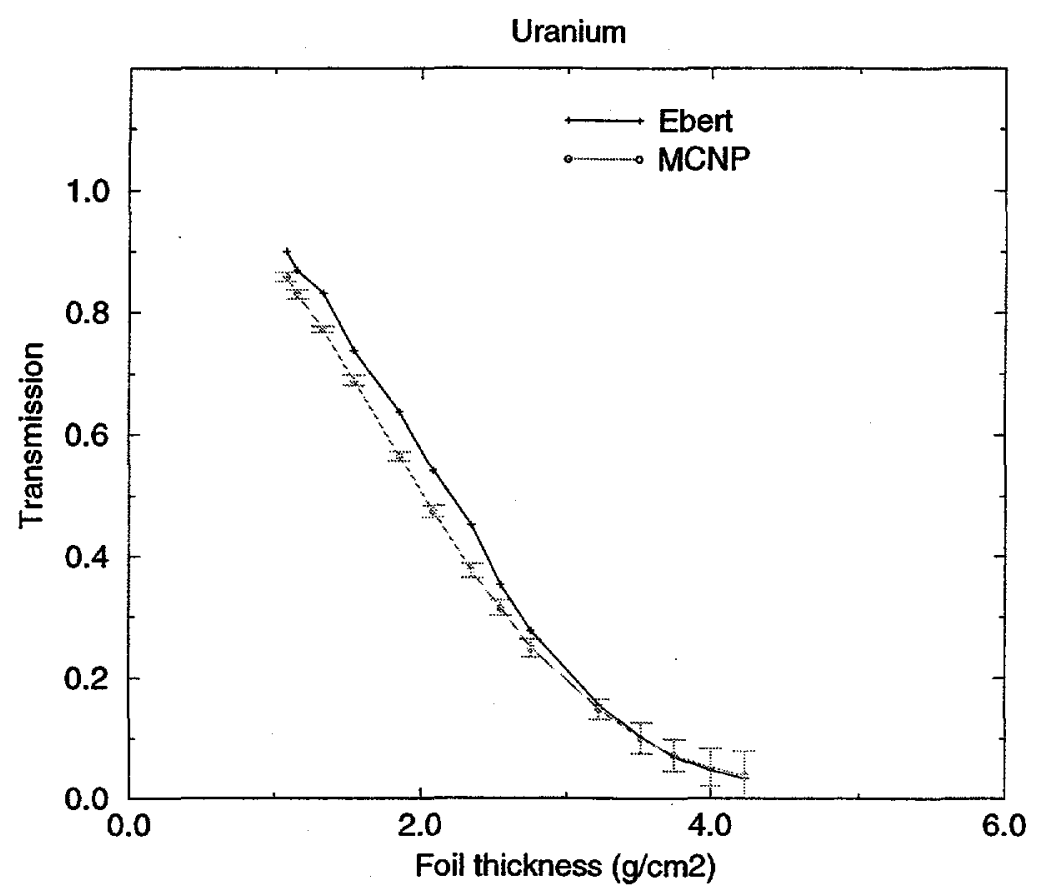

Fig. 17. Comparison of transmission coefficients for $10.2 \mathrm{MeV}$ electrons incident on $\mathrm{U}$ foils.

TABLE VII: ELECTRON SATURATION BACKSCATTER COMPARISON

\begin{tabular}{|c|c|c|c|c|c|}
\hline Material & $\begin{array}{c}\text { Electron } \\
\text { Energy } \\
(\mathrm{MeV})\end{array}$ & Ebert & Dressel & Tabata & MCNP \\
\hline \hline $\mathrm{C}$ & & $4.0 \mathrm{E}-03$ & $9.0 \mathrm{E}-03$ & $3.20 \mathrm{E}-03$ & $5.846 \mathrm{E}-03(4.0)^{*}$ \\
$\mathrm{Ag}$ & 10.2 & $7.4 \mathrm{E}-02$ & $1.8 \mathrm{E}-01$ & $7.35 \mathrm{E}-02$ & $8.465 \mathrm{E}-02(3.0)$ \\
$\mathrm{U}$ & & $1.47 \mathrm{E}-01$ & $3.3 \mathrm{E}-01$ & $1.36 \mathrm{E}-01$ & $1.780 \mathrm{E}-01(2.0)$ \\
\hline $\mathrm{C}$ & & $5.0 \mathrm{E}-03$ & $8.6 \mathrm{E}-03$ & $4.00 \mathrm{E}-03$ & $6.122 \mathrm{E}-03(4.0)$ \\
$\mathrm{Ag}$ & 8.0 & $9.5 \mathrm{E}-02$ & $2.0 \mathrm{E}-01$ & $9.70 \mathrm{E}-02$ & $1.149 \mathrm{E}-01(2.0)$ \\
$\mathrm{U}$ & & $1.95 \mathrm{E}-01$ & $3.8 \mathrm{E}-01$ & $1.72 \mathrm{E}-01$ & $2.179 \mathrm{E}-01(2.0)$ \\
\hline $\mathrm{C}$ & & $6.0 \mathrm{E}-03$ & $1.0 \mathrm{E}-02$ & $5.00 \mathrm{E}-03$ & $6.856 \mathrm{E}-03(3.0)$ \\
$\mathrm{Ag}$ & 6.0 & $1.39 \mathrm{E}-01$ & $2.4 \mathrm{E}-01$ & $1.29 \mathrm{E}-02$ & $1.504 \mathrm{E}-01(2.0)$ \\
$\mathrm{U}$ & & $2.45 \mathrm{E}-01$ & $4.5 \mathrm{E}-01$ & $2.28 \mathrm{E}-01$ & $2.780 \mathrm{E}-01(1.01)$ \\
\hline
\end{tabular}

$*_{\text {read as }} 5.846 \times 10^{-3}$ with $4.0 \%$ error 


\section{PHYSICS PARAMETER STUDIES}

The main purpose of this section is to present a systematic study of the impact of varying physics parameters on the calculational results. The variations show that the results change in the expected way and show where the calculation is sensitive to the choice of model, indicating possibly fruitful areas of further research and code development. This set of simulations explore several physics and variance reduction models for the experiments of Faddegon et al., ${ }^{6,7}$ described in Section II.A.1, and Ebert et al., ${ }^{11}$ described in Section III.A.1. The effect of varying several of the physics parameters for electron transport is also explored for calculations of depth dose in a water disk.

\section{A. Bremsstrahlung Angular Models}

In order to study the impact of different physics models for the angular distribution of bremsstrahlung photons, the MCNP electron physics parameter IBAD was varied in several simulations of Faddegon's work. Changing IBAD from its default of 0 to 1 switches the detailed bremsstrahlung sampling to a simple approximation. For the detailed treatment of bremsstrahlung, MCNP primarily uses the Bethe-Heitler ${ }^{13}$ Born approximation, based on some of the formulas given in Koch and Motz. ${ }^{17}$ The simple probability distribution, invoked when IBAD=1, is given by

$$
p(\mu) d \mu=\frac{1-\beta^{2}}{2(1-\beta \mu)^{2}} d \mu
$$

where $\mu=\cos \theta$ and $\beta=v / c$. This simple sampling method is always used for detectors, regardless of the value of IBAD. Specifying the generation of bremsstrahlung photons to be based on Eq. (7) forces the actual transport to be consistent with detector contributions.

Figure 18 shows the results of the IBAD comparisons for lead. The experimental results are shown with the MCNP4B results for default and IBAD=1 cell tallies, and default detector tallies. These data are also given in Table VIII. The numbers in parentheses are percent errors. As expected, the IBAD $=1$ cell tallies track the detector tallies. Changing IBAD produces no statistically significant difference between detector tallies because detector tallies always use the simple bremsstrahlung sampling, regardless of the value of IBAD, and for these problems, which are 
photon thin, the major detector contribution is from first generation bremsstrahlung photons that undergo very little scatter in the problem. The cell tallies for forward angles increase for the case $\mathrm{IBAD}=1$. The detector tallies for these angles are usually greater than default cell tallies as well. The effect of $\mathrm{BBAD}$ decreases as the angle increases. Tables $\mathrm{IX}$ and $\mathrm{X}$ give results for the analogous simulations for $\mathrm{Al}$ and $\mathrm{Be}$. The results are also plotted in Figs. 19 and 20. The same trends that were seen in MCNP4B simulations were seen with MCNP4A IBAD comparisons, which are given for lead and aluminum in Tables XI and XII.

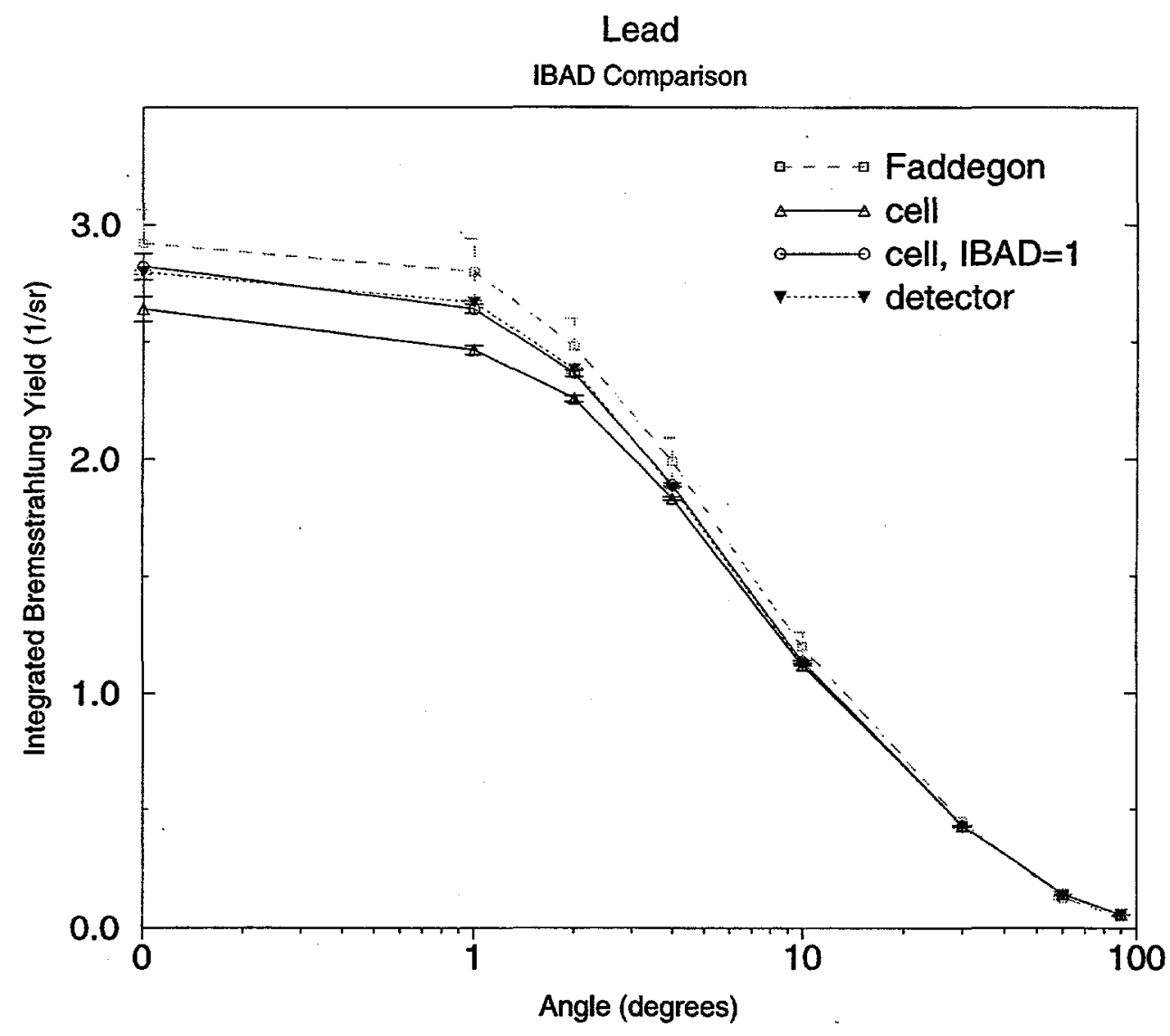

Fig. 18. Integrated bremsstrahlung yield IBAD comparison for lead. 
TABLE VIII: PB INTEGRATED BREMSSTRAHLUNG YLELD, IBAD COMPARISON

\begin{tabular}{|c|c|c|c|c|c|}
\hline Angle & $\begin{array}{c}\text { 4B } \\
\text { Cell }\end{array}$ & $\begin{array}{c}\text { 4B } \\
\text { Detector }\end{array}$ & $\begin{array}{c}\text { 4B } \\
\text { Cell } \\
\text { IBAD=1 }\end{array}$ & $\begin{array}{c}\text { 4B } \\
\text { Detector } \\
\text { IBAD=1 }\end{array}$ & Faddegon \\
\hline \hline 0 & $2.640 \mathrm{E}+00(2.0)^{*}$ & $2.797 \mathrm{E}+00(0.3)$ & $2.822 \mathrm{E}+00(2.0)$ & $2.799 \mathrm{E}+00(0.3)$ & $2.92 \mathrm{E}+00(5.0)$ \\
1 & $2.463 \mathrm{E}+00(0.8)$ & $2.667 \mathrm{E}+00(0.3)$ & $2.641 \mathrm{E}+00(0.7)$ & $2.669 \mathrm{E}+00(0.3)$ & $2.80 \mathrm{E}+00(5.0)$ \\
2 & $2.255 \mathrm{E}+00(0.6)$ & $2.380 \mathrm{E}+00(0.3)$ & $2.364 \mathrm{E}+00(0.6)$ & $2.381 \mathrm{E}+00(0.3)$ & $2.48 \mathrm{E}+00(5.0)$ \\
4 & $1.835 \mathrm{E}+00(0.4)$ & $1.883 \mathrm{E}+00(0.3)$ & $1.892 \mathrm{E}+00(0.4)$ & $1.885 \mathrm{E}+00(0.3)$ & $1.99 \mathrm{E}+00(5.0)$ \\
10 & $1.122 \mathrm{E}+00(0.4)$ & $1.125 \mathrm{E}+00(0.3)$ & $1.136 \mathrm{E}+00(0.4)$ & $1.127 \mathrm{E}+00(0.3)$ & $1.2 \mathrm{E}+00(5.0)$ \\
30 & $4.338 \mathrm{E}-01(0.4)$ & $4.310 \mathrm{E}-01(0.5)$ & $4.337 \mathrm{E}-01(0.3)$ & $4.316 \mathrm{E}-01(0.4)$ & $4.47 \mathrm{E}-01(5.0)$ \\
60 & $1.444 \mathrm{E}-01(0.5)$ & $1.434 \mathrm{E}-01(0.5)$ & $1.433 \mathrm{E}-01(0.5)$ & $1.436 \mathrm{E}-01(0.5)$ & $1.29 \mathrm{E}-01(5.0)$ \\
90 & $6.029 \mathrm{E}-02(0.7)$ & $5.860 \mathrm{E}-02(0.7)$ & $5.999 \mathrm{E}-02(0.7)$ & $5.852 \mathrm{E}-02(0.6)$ & $5.19 \mathrm{E}-02(7.0)$ \\
\hline
\end{tabular}

$*_{\text {read as }} 2.640 \times 10^{0}$ with $2.0 \%$ error

TABLE IX: AL INTEGRATED BREMSSTRAHLUNG YIELD, IBAD COMPARISON

\begin{tabular}{|c|c|c|c|c|c|}
\hline Angle & $\begin{array}{c}\text { 4B } \\
\text { Cell }\end{array}$ & $\begin{array}{c}\text { 4B } \\
\text { Detector }\end{array}$ & $\begin{array}{c}\text { 4B } \\
\text { Cell } \\
\text { IBAD=1 }\end{array}$ & $\begin{array}{c}\text { 4B } \\
\text { Detector } \\
\text { IBAD=1 }\end{array}$ & Faddegon \\
\hline \hline 0 & $2.991 \mathrm{E}+00(1.5)^{*}$ & $3.224 \mathrm{E}+00(0.2)$ & $3.245 \mathrm{E}+00(1.5)$ & $3.225 \mathrm{E}+00(0.2)$ & $3.42 \mathrm{E}+00(5.0)$ \\
1 & $2.802 \mathrm{E}+00(0.6)$ & $3.045 \mathrm{E}+00(0.2)$ & $3.013 \mathrm{E}+00(0.5)$ & $3.044 \mathrm{E}+00(0.2)$ & $3.21 \mathrm{E}+00(5.0)$ \\
2 & $2.494 \mathrm{E}+00(0.4)$ & $2.670 \mathrm{E}+00(0.2)$ & $2.657 \mathrm{E}+00(0.4)$ & $2.670 \mathrm{E}+00(0.2)$ & $2.78 \mathrm{E}+00(5.0)$ \\
4 & $1.931 \mathrm{E}+00(0.3)$ & $2.009 \mathrm{E}+00(0.2)$ & $2.007 \mathrm{E}+00(0.3)$ & $2.010 \mathrm{E}+00(0.2)$ & $2.14 \mathrm{E}+00(5.0)$ \\
10 & $1.033 \mathrm{E}+00(0.3)$ & $1.043 \mathrm{E}+00(0.2)$ & $1.044 \mathrm{E}+00(0.3)$ & $1.044 \mathrm{E}+00(0.2)$ & $1.06 \mathrm{E}+00(5.0)$ \\
30 & $2.664 \mathrm{E}-01(0.3)$ & $2.673 \mathrm{E}-01(0.3)$ & $2.665 \mathrm{E}-01(0.3)$ & $2.674 \mathrm{E}-01(0.3)$ & $2.65 \mathrm{E}-01(5.0)$ \\
60 & $7.284 \mathrm{E}-02(0.5)$ & $7.278 \mathrm{E}-02(0.3)$ & $7.293 \mathrm{E}-02(0.5)$ & $7.261 \mathrm{E}-02(0.3)$ & $6.66 \mathrm{E}-02(6.0)$ \\
90 & $3.294 \mathrm{E}-02(0.7)$ & $3.280 \mathrm{E}-02(0.3)$ & $3.285 \mathrm{E}-02(0.7)$ & $3.269 \mathrm{E}-02(0.3)$ & $2.87 \mathrm{E}-02(6.0)$ \\
\hline
\end{tabular}

$*_{\text {read as }} 2.991 \times 10^{\circ}$ with $1.5 \%$ error 
TABLE X: BE INTEGRATED BREMSSTRAHLUNG YIELD, IBAD COMPARISON

\begin{tabular}{|c|c|c|c|c|c|}
\hline Angle & $\begin{array}{c}\text { 4B } \\
\text { Cell }\end{array}$ & $\begin{array}{c}\text { 4B } \\
\text { Detector }\end{array}$ & $\begin{array}{c}\text { 4B } \\
\text { Cell } \\
\text { IBAD=1 }\end{array}$ & $\begin{array}{c}\text { 4B } \\
\text { Detector } \\
\text { IBAD=1 }\end{array}$ & Faddegon \\
\hline \hline 0 & $2.332 \mathrm{E}+00(1.8)^{*}$ & $2.588 \mathrm{E}+00(0.2)$ & $2.557 \mathrm{E}+00(1.8)$ & $2.587 \mathrm{E}+00(0.3)$ & $2.73 \mathrm{E}+00(5.1)$ \\
1 & $2.176 \mathrm{E}+00(0.7)$ & $2.412 \mathrm{E}+00(0.2)$ & $2.395 \mathrm{E}+00(0.7)$ & $2.411 \mathrm{E}+00(0.3)$ & $2.57 \mathrm{E}+00(5.1)$ \\
2 & $1.917 \mathrm{E}+00(0.5)$ & $2.058 \mathrm{E}+00(0.2)$ & $2.034 \mathrm{E}+00(0.5)$ & $2.059 \mathrm{E}+00(0.2)$ & $2.14 \mathrm{E}+00(5.1)$ \\
4 & $1.390 \mathrm{E}+00(0.4)$ & $1.444 \mathrm{E}+00(0.2)$ & $1.439 \mathrm{E}+00(0.4)$ & $1.445 \mathrm{E}+00(0.2)$ & $1.54 \mathrm{E}+00(5.0)$ \\
10 & $5.977 \mathrm{E}-01(0.4)$ & $6.030 \mathrm{E}-01(0.3)$ & $6.023 \mathrm{E}-01(0.4)$ & $6.036 \mathrm{E}-01(0.3)$ & $6.30 \mathrm{E}-01(5.1)$ \\
30 & $9.368 \mathrm{E}-02(0.6)$ & $9.195 \mathrm{E}-02(0.3)$ & $9.214 \mathrm{E}-02(0.6)$ & $9.202 \mathrm{E}-02(0.4)$ & $9.49 \mathrm{E}-02(5.1)$ \\
60 & $2.294 \mathrm{E}-02(0.9)$ & $2.265 \mathrm{E}-02(0.3)$ & $2.251 \mathrm{E}-02(0.9)$ & $2.261 \mathrm{E}-02(0.3)$ & $2.38 \mathrm{E}-02(5.9)$ \\
90 & $1.079 \mathrm{E}-02(1.2)$ & $1.053 \mathrm{E}-02(0.3)$ & $1.044 \mathrm{E}-02(1.3)$ & $1.048 \mathrm{E}-02(0.3)$ & $1.06 \mathrm{E}-02(7.0)$ \\
\hline
\end{tabular}

$*_{\text {read as }} 2.332 \times 10^{0}$ with $1.8 \%$ error

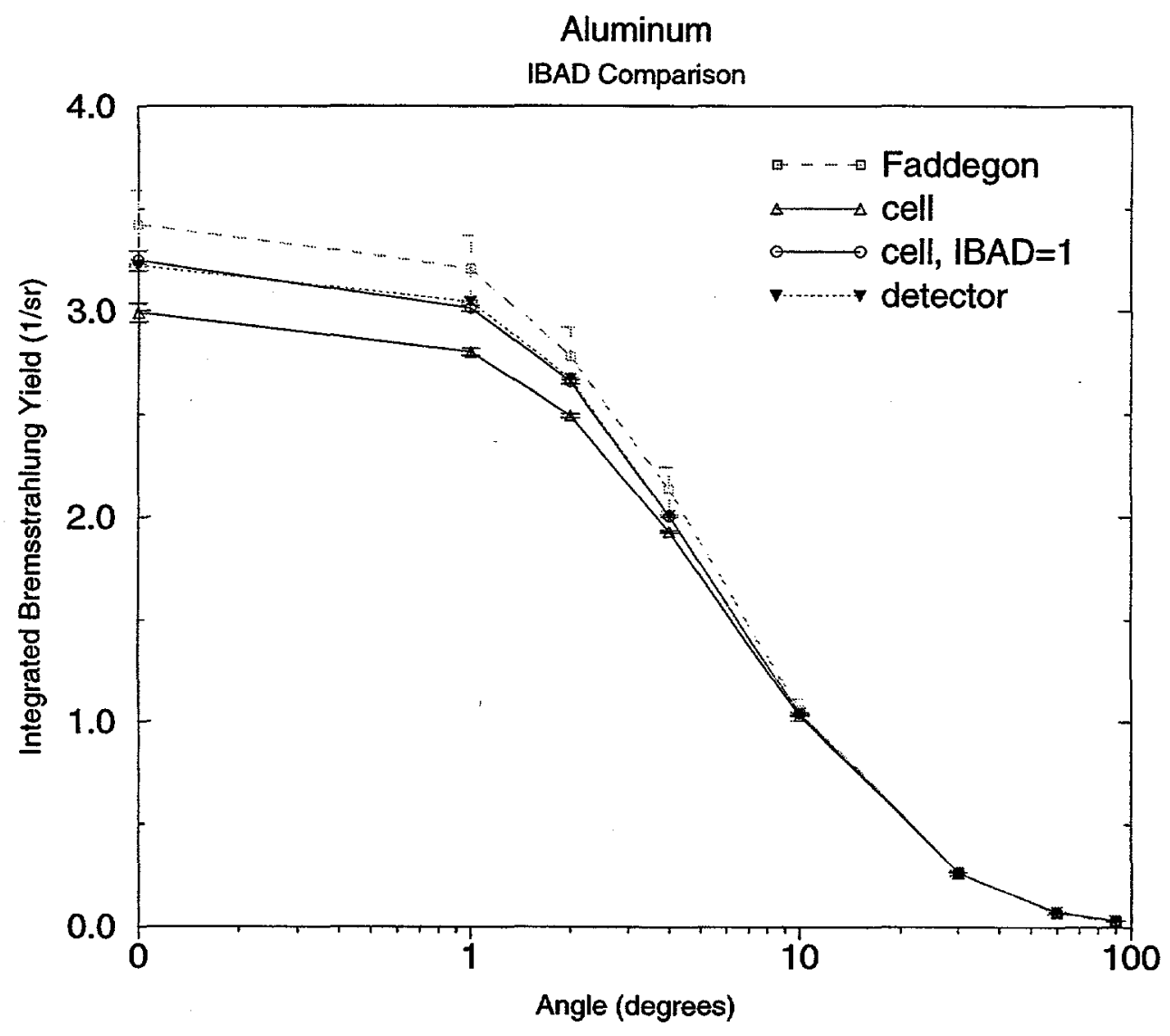

Fig. 19. IBAD comparison for aluminum. 


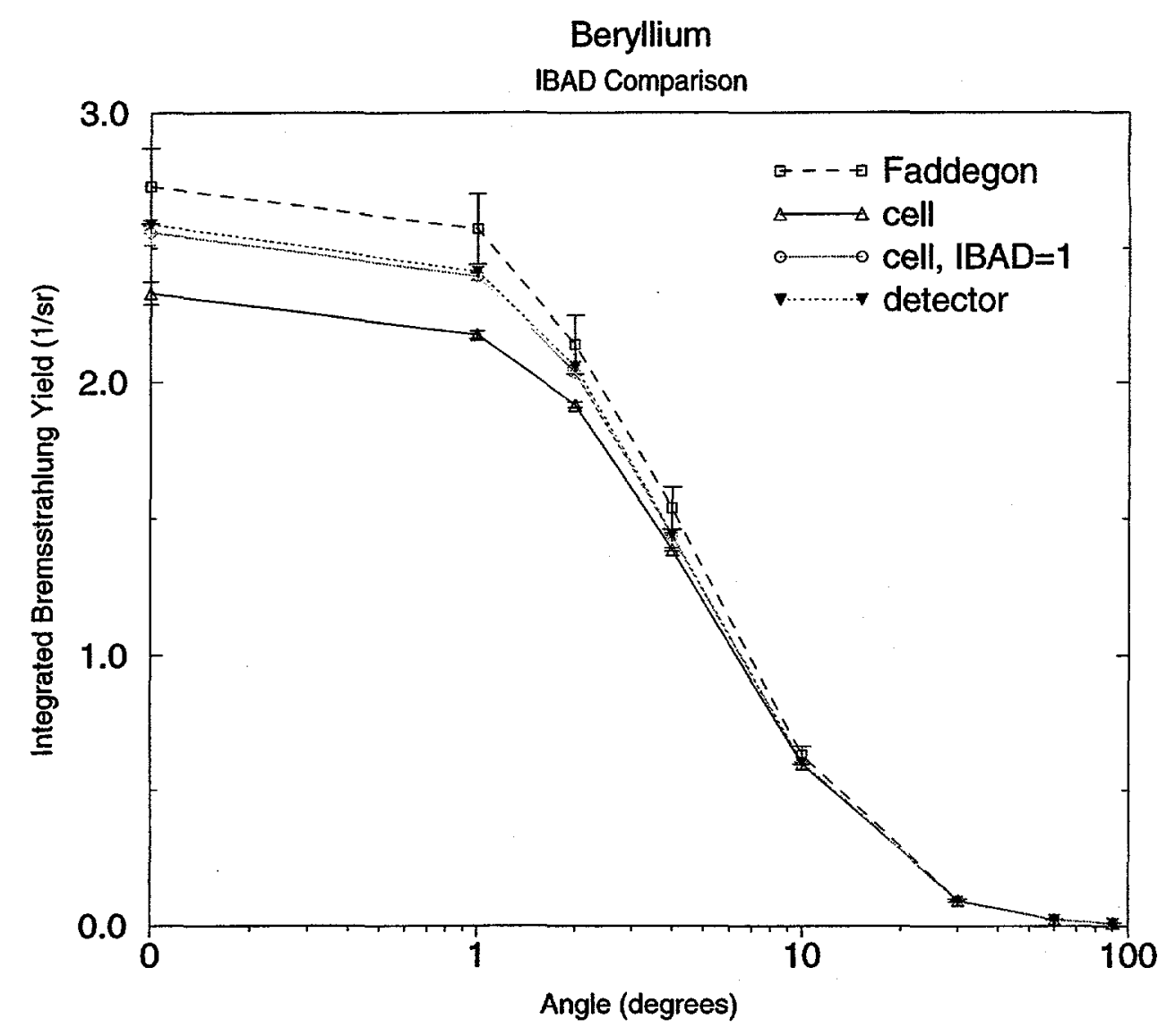

Fig. 20. IBAD comparison for beryllium.

TABLE XI: PB INTEGRATED BREMSSTRAHLUNG YIELD, MCNP4A IBAD COMPARISON

\begin{tabular}{|c|c|c|c|c|c|}
\hline Angle & $\begin{array}{c}\text { 4A } \\
\text { Cell }\end{array}$ & $\begin{array}{c}\text { 4A } \\
\text { Detector }\end{array}$ & $\begin{array}{c}\text { 4A } \\
\text { Cell } \\
\text { IBAD=1 }\end{array}$ & $\begin{array}{c}\text { 4A } \\
\text { Detector } \\
\text { IBAD=1 }\end{array}$ & Faddegon \\
\hline \hline 0 & $3.338 \mathrm{E}+00(2.7)^{*}$ & $3.356 \mathrm{E}+00(0.5)$ & $3.412 \mathrm{E}+00(3.1)$ & $3.349 \mathrm{E}+00(0.5)$ & $2.92 \mathrm{E}+00(5.0)$ \\
1 & $2.650 \mathrm{E}+00(1.1)$ & $2.914 \mathrm{E}+00(0.4)$ & $2.933 \mathrm{E}+00(1.1)$ & $2.916 \mathrm{E}+00(0.5)$ & $2.80 \mathrm{E}+00(5.0)$ \\
2 & $2.297 \mathrm{E}+00(0.8)$ & $2.413 \mathrm{E}+00(0.4)$ & $2.411 \mathrm{E}+00(0.9)$ & $2.414 \mathrm{E}+00(0.5)$ & $2.48 \mathrm{E}+00(5.0)$ \\
4 & $1.827 \mathrm{E}+00(0.6)$ & $1.884 \mathrm{E}+00(0.4)$ & $1.896 \mathrm{E}+00(0.7)$ & $1.889 \mathrm{E}+00(0.5)$ & $1.99 \mathrm{E}+00(5.0)$ \\
10 & $1.134 \mathrm{E}+00(0.5)$ & $1.140 \mathrm{E}+00(0.5)$ & $1.133 \mathrm{E}+00(0.6)$ & $1.138 \mathrm{E}+00(0.6)$ & $1.2 \mathrm{E}+00(5.0)$ \\
30 & $4.369 \mathrm{E}-01(0.5)$ & $4.374 \mathrm{E}-01(0.6)$ & $4.410 \mathrm{E}-01(0.6)$ & $4.375 \mathrm{E}-01(0.7)$ & $4.47 \mathrm{E}-01(5.0)$ \\
60 & $1.470 \mathrm{E}-01(0.6)$ & $1.464 \mathrm{E}-01(0.7)$ & $1.458 \mathrm{E}-01(0.8)$ & $1.476 \mathrm{E}-01(0.8)$ & $1.29 \mathrm{E}-01(5.0)$ \\
90 & $6.107 \mathrm{E}-02(0.9)$ & $6.028 \mathrm{E}-02(0.9)$ & $6.026 \mathrm{E}-02(1.0)$ & $6.015 \mathrm{E}-02(0.9)$ & $5.19 \mathrm{E}-02(7.0)$ \\
\hline
\end{tabular}

*read as $3.338 \times 10^{\circ}$ with $2.7 \%$ error 
TABLE XII: AL INTEGRATED BREMSSTRAHLUNG YIELD, MCNP4A IBAD COMPARISON

\begin{tabular}{|c|c|c|c|c|c|}
\hline Angle & $\begin{array}{c}\text { 4A } \\
\text { Cell }\end{array}$ & $\begin{array}{c}\text { 4A } \\
\text { Detector }\end{array}$ & $\begin{array}{c}\text { 4A } \\
\text { Cell } \\
\text { IBAD=1 }\end{array}$ & $\begin{array}{c}\text { 4A } \\
\text { Detector } \\
\text { IBAD=1 }\end{array}$ & Faddegon \\
\hline \hline 0 & $3.744 \mathrm{E}+00(2.0)^{*}$ & $3.959 \mathrm{E}+00(0.3)$ & $3.837 \mathrm{E}+00(1.7)$ & $3.965 \mathrm{E}+00(0.3)$ & $3.42 \mathrm{E}+00(5.0)$ \\
1 & $3.042 \mathrm{E}+00(0.7)$ & $3.368 \mathrm{E}+00(0.3)$ & $3.318 \mathrm{E}+00(0.6)$ & $3.373 \mathrm{E}+00(0.3)$ & $3.21 \mathrm{E}+00(5.0)$ \\
2 & $2.522 \mathrm{E}+00(0.6)$ & $2.695 \mathrm{E}+00(0.3)$ & $2.698 \mathrm{E}+00(0.5)$ & $2.698 \mathrm{E}+00(0.2)$ & $2.78 \mathrm{E}+00(5.0)$ \\
4 & $1.942 \mathrm{E}+00(0.4)$ & $2.009 \mathrm{E}+00(0.3)$ & $2.015 \mathrm{E}+00(0.4)$ & $2.009 \mathrm{E}+00(0.3)$ & $2.14 \mathrm{E}+00(5.0)$ \\
10 & $1.045 \mathrm{E}+00(0.4)$ & $1.057 \mathrm{E}+00(0.3)$ & $1.060 \mathrm{E}+00(0.4)$ & $1.056 \mathrm{E}+00(0.3)$ & $1.06 \mathrm{E}+00(5.0)$ \\
30 & $2.723 \mathrm{E}-01(0.4)$ & $2.710 \mathrm{E}-01(0.3)$ & $2.733 \mathrm{E}-01(0.4)$ & $2.712 \mathrm{E}-01(0.3)$ & $2.65 \mathrm{E}-01(5.0)$ \\
60 & $7.416 \mathrm{E}-02(0.6)$ & $7.408 \mathrm{E}-02(0.3)$ & $7.404 \mathrm{E}-02(0.6)$ & $7.397 \mathrm{E}-02(0.3)$ & $6.66 \mathrm{E}-02(6.0)$ \\
90 & $3.367 \mathrm{E}-02(0.8)$ & $3.362 \mathrm{E}-02(0.4)$ & $3.343 \mathrm{E}-02(0.8)$ & $3.353 \mathrm{E}-02(0.4)$ & $2.87 \mathrm{E}-02(6.0)$ \\
\hline
\end{tabular}

*read as $3.744 \times 10^{\circ}$ with $2.0 \%$ error

\section{B. Coherent Scatter}

Since the coherent scattering (Thomson) cross section can be forward peaked at high energies, with no energy loss, it is as if no scattering took place. For point detector tallies to sample coherent scattering, the point must be on the original particle track, which usually does not occur. Including coherent scatter can lead to larger variances in detector tallies. Coherent scatter is included by default in the detailed physics photon transport in MCNP; it can be turned off by setting the NOCOH entry of the PHYS:P card in the input file to 1.

The impact of coherent scatter on tally results was examined further. Table XIII shows the integrated yields for lead at a few angles. The yields for angles less than $30^{\circ}$ can not be compared to experiment because the simulations did not include the stainless steel entrance window (see Section IV.A.1). The geometry for the two simulations were, however, consistent with each other. The number of histories did differ between the two simulations, so the statistical errors cannot be directly compared. Figure 21 compares the bremsstrahlung spectra at $10^{\circ}$ for detector tallies with and without coherent scatter. Table XIII and Fig. 21 show that coherent scatter does not significantly affect either the bremsstrahlung spectra or the integrated yield. Coherent scatter can, however, affect the statistical convergence of detector tallies. The detector tallies without coherent scatter passed the MCNP statistical checks for tally convergence much sooner than the simulation with coherent scatter. This is because including coherent scatter can lead to very large detector tally scores, and thus a higher variance. These large scores were verified by a separate calculation, so MCNP is behaving as expected. 
TABLE XIII: PB INTEGRATED BREMSSTRAHLUNG YIELD, COHERENT SCATTER COMPARISON

\begin{tabular}{|c|c|c|c|c|}
\hline Angle & $\begin{array}{c}\text { 4B } \\
\text { Cell }\end{array}$ & $\begin{array}{c}\text { 4B } \\
\text { Cell } \\
\text { no coherent scatter }\end{array}$ & $\begin{array}{c}\text { 4B } \\
\text { Detector }\end{array}$ & $\begin{array}{c}\text { 4B } \\
\text { Detector } \\
\text { no coherent } \\
\text { scatter }\end{array}$ \\
\hline \hline 0 & $2.717 \mathrm{E}+00(2.2)^{*}$ & $2.853 \mathrm{E}+00(3.7)$ & $2.811 \mathrm{E}+00(0.3)$ & $2.802 \mathrm{E}+00(0.5)$ \\
1 & $2.509 \mathrm{E}+00(0.8)$ & $2.464 \mathrm{E}+00(1.4)$ & $2.681 \mathrm{E}+00(0.3)$ & $2.669 \mathrm{E}+00(0.5)$ \\
2 & $2.280 \mathrm{E}+00(0.6)$ & $2.278 \mathrm{E}+00(1.0)$ & $2.401 \mathrm{E}+00(0.3)$ & $2.385 \mathrm{E}+00(0.5)$ \\
4 & $1.836 \mathrm{E}+00(0.5)$ & $1.846 \mathrm{E}+00(0.8)$ & $1.901 \mathrm{E}+00(0.3)$ & $1.926 \mathrm{E}+00(0.5)$ \\
10 & $1.133 \mathrm{E}+00(0.4)$ & $1.131 \mathrm{E}+00(0.7)$ & $1.137 \mathrm{E}+00(0.4)$ & $1.146 \mathrm{E}+00(0.6)$ \\
30 & $4.338 \mathrm{E}-01(0.4)$ & $4.341 \mathrm{E}-01(0.6)$ & $4.310 \mathrm{E}-01(0.5)$ & $4.315 \mathrm{E}-01(0.8)$ \\
60 & $1.444 \mathrm{E}-01(0.5)$ & $1.438 \mathrm{E}-01(0.8)$ & $1.434 \mathrm{E}-01(0.5)$ & $1.450 \mathrm{E}-01(0.9)$ \\
90 & $6.029 \mathrm{E}-02(0.7)$ & $6.106 \mathrm{E}-02(1.2)$ & $5.860 \mathrm{E}-02(0.7)$ & $5.959 \mathrm{E}-02(1.0)$ \\
\hline
\end{tabular}

$*_{\text {read }}$ as $2.717 \times 10^{0}$ with $2.2 \%$ error

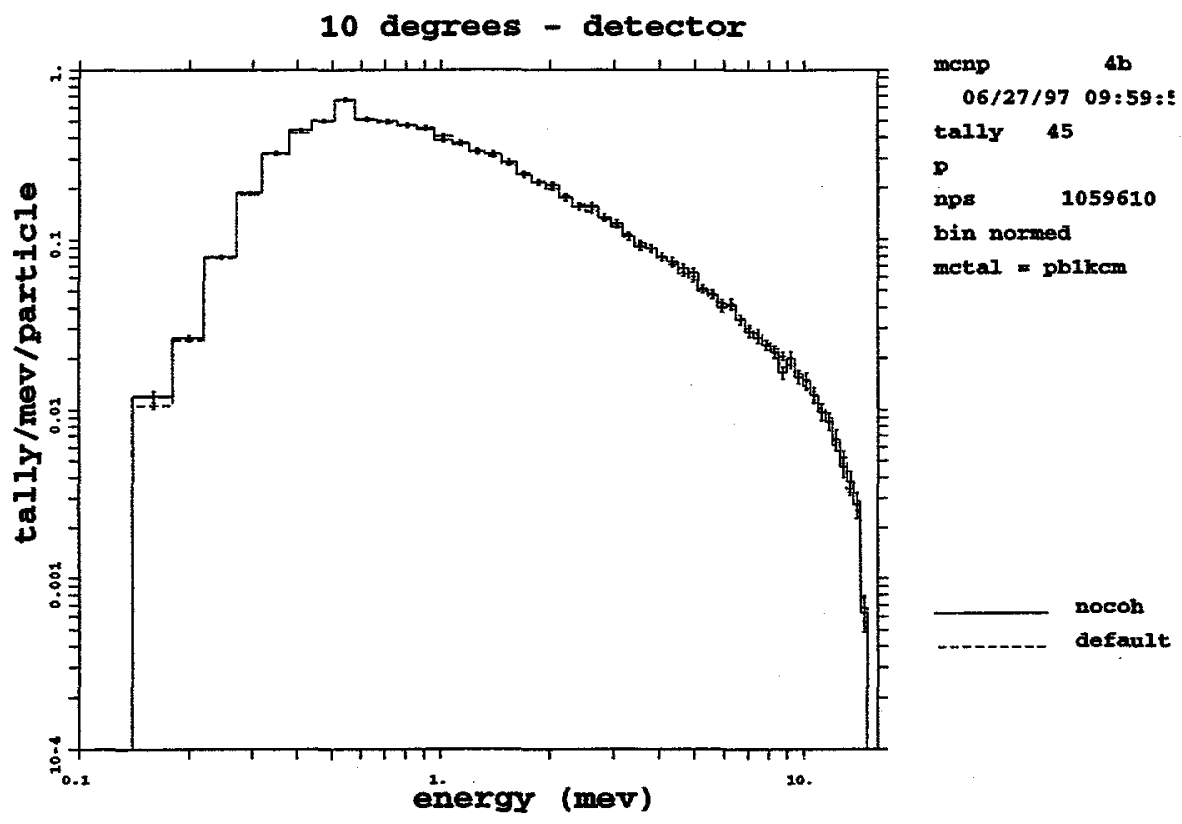

Fig. 21. Coherent scatter comparison for lead, detector tally. 


\section{Electron Sub-Step Size}

The ESTEP parameter, which sets the number of electron substeps per energy step, was also varied for the pre-target material in order to test the existence of bremsstrahlung step size sampling artifacts. ${ }^{3}$ Very thin material regions such as the pre-target Ti exit window, Si detector, and stainless steel target chamber present in this problem may not allow enough electron substeps for an accurate simulation of the electron's trajectory. One of the improvements in MCNP4B over MCNP4A was the mitigation of an electron sub-step artifact for bremsstrahlung production. Comparisons of versions $4 \mathrm{~B}$ and $4 \mathrm{~A}$ are presented next.

For MCNP4A, it has been reported that increasing ESTEP will eliminate artificially high bremsstrahlung yields for forward angles. ${ }^{5}$ Figure 22 illustrates the effect of increasing ESTEP for two arbitrary electron tracks. In MCNP4A, using the default sub-step size, a bremsstrahlung photon will be sampled either at position 1 or 2 , with direction $\Omega_{1}$ or $\Omega_{2}$. This means that the photon angular distribution will be very forward peaked, since $50 \%$ of the photons will be in the forward direction. For the case with the increased ESTEP, the same number of photons would be generated, but only $1 / 7$ would be in the forward direction, since any one of $\Omega_{1}$ through $\Omega_{7}$ can be sampled. Increasing ESTEP in MCNP4A, therefore, severely impacted the production of bremsstrahlung photons. In MCNP4B, the bremsstrahlung sampling is different in that the code chooses to generate a photon somewhere along the electron substep, and the photon angle is prorated according to how far along the substep the photon has been sampled. This sampling will lead to less of a dependence on sub-step size for bremsstrahlung production.

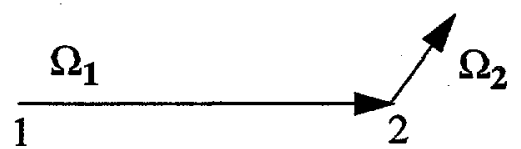

Default ESTEP

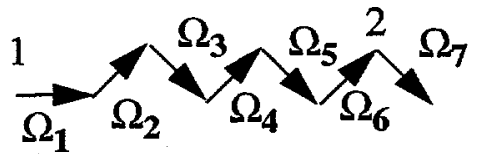

6X Default ESTEP

Fig. 22. Increasing ESTEP

The ESTEP parameter was varied for $\mathrm{Al}$ and $\mathrm{Pb}$ target geometries. Tables $\mathrm{XIV}$ and $\mathrm{XV}$ illustrate the effect of changing ESTEP with MCNP4A and MCNP4B for Al. Note that in MCNP4A, the results appear to converge to the experimental result for an ESTEP of 6 times the 
default. Increasing the ESTEP parameter to 60 times the default value, however, continues to lower the yield. Increasing ESTEP for MCNP4A, therefore, does not allow the user to approach the correct answer, unless some value of ESTEP that gives a correct answer is chosen by chance. This effect is not seen to such a great extent in MCNP4B because the bremsstrahlung sampling scheme has been modified as described above. For example, when comparing the default and 60 times default ESTEP values, the $0^{\circ}$ tallies differ by about $40 \%$ for MCNP4A, but only by about 7\% for version 4B. This difference is statistically significant and suggests the need for further study but is a great improvement.

Figure 23 shows the ratio of calculation to experiment for the $\mathrm{Al} 0^{\circ}$ cell tally for a few multiples of the default ESTEP value. Note how MCNP4B is much less sensitive to ESTEP than MCNP4A.

TABLE XIV: MCNP4A ESTEP COMPARISON FOR AL

\begin{tabular}{|c|c|c|c|c|c|c|c|}
\hline Angle & $\begin{array}{l}4 \mathrm{~A} \\
\text { Cell }\end{array}$ & $\begin{array}{c}4 \mathrm{~A} \\
\text { Detector }\end{array}$ & $\begin{array}{c}\text { 4A } \\
\text { Cell } \\
6 \mathrm{X} \text { ESTEP }\end{array}$ & $\begin{array}{c}\text { 4A } \\
\text { Detector } \\
6 \mathrm{X} \text { ESTEP }\end{array}$ & $\begin{array}{c}4 A \\
\text { Cell } \\
60 \mathrm{X} \text { ESTEP }\end{array}$ & $\begin{array}{c}\text { 4A } \\
\text { Detector } \\
60 \mathrm{X} \text { ESTEP }\end{array}$ & Faddegon \\
\hline 0 & $3.744 \mathrm{E}+00(2.0)^{*}$ & $3.959 \mathrm{E}+00(0.3)$ & $3.345 \mathrm{E}+00(3.0)$ & $3.392 \mathrm{E}+00(0.5)$ & $2.080 \mathrm{E}+00(3.2)$ & $2.281 \mathrm{E}+00(0.4)$ & $3.42 \mathrm{E}+00(5.0)$ \\
\hline 1 & $3.042 E+00(0.7)$ & $3.368 \mathrm{E}+00(0.3)$ & $2.855 \mathrm{E}+00(1.2)$ & $3.135 \mathrm{E}+00(0.4)$ & $2.099 E+00(1.1)$ & $2.201 E+00(0.4)$ & $3.21 \mathrm{E}+00(5.0)$ \\
\hline 2 & $2.522 \mathrm{E}+00(0.6)$ & $2.695 \mathrm{E}+00(0.3)$ & $2.513 E+00(0.9)$ & $2.681 \mathrm{E}+00(0.4)$ & $1.946 E+00(0.8)$ & $2.025 \mathrm{E}+00(0.4)$ & $2.78 \mathrm{E}+00(5.0)$ \\
\hline 4 & $1.942 E+00(0.4)$ & $2.009 E+00(0.3)$ & $1.954 \mathrm{E}+00(0.7)$ & $2.039 \mathrm{E}+00(0.4)$ & $1.680 \mathrm{E}+00(0.6)$ & $1.731 E+00(0.4)$ & $2.14 E+00(5.0)$ \\
\hline 10 & $1.045 \mathrm{E}+00(0.4)$ & $1.057 \mathrm{E}+00(0.3)$ & $1.043 E+00(0.6)$ & $1.061 E+00(0.5)$ & $1.050 \mathrm{E}+00(0.5)$ & $1.067 \mathrm{E}+00(0.4)$ & $1.06 \mathrm{E}+00(5.0)$ \\
\hline 30 & $2.723 \mathrm{E}-01(0.4)$ & $2.710 \mathrm{E}-01(0.3)$ & $2.722 \mathrm{E}-01(0.6)$ & $2.707 \mathrm{E}-01(0.5)$ & $2.745 \mathrm{E}-01(0.6)$ & $2.782 \mathrm{E}-01(0.5)$ & $2.65 \mathrm{E}-01(5.0)$ \\
\hline 60 & $7.416 \mathrm{E}-02(0.6)$ & $7.408 \mathrm{E}-02(0.3)$ & $7.532 \mathrm{E}-02(0.8)$ & $7.365 \mathrm{E}-02(0.5)$ & $7.615 \mathrm{E}-02(0.8)$ & $7.474 \mathrm{E}-02(0.5)$ & $6.66 \mathrm{E}-02(6.0)$ \\
\hline 90 & $3.367 \mathrm{E}-02(0.8)$ & $3.362 \mathrm{E}-02(0.4)$ & $3.399 \mathrm{E}-02(1.1)$ & $3.360 \mathrm{E}-02(0.5)$ & $3.374 \mathrm{E}-02(1.2)$ & $3.370 \mathrm{E}-02(0.5)$ & $2.87 \mathrm{E}-02(6.0)$ \\
\hline
\end{tabular}

$*_{\text {read as }} 3.744 \times 10^{\circ}$ with $2.0 \%$ error

TABLE XV: MCNP4B ESTEP COMPARISON FOR AL

\begin{tabular}{|c|c|c|c|c|c|c|c|}
\hline Angle & $\begin{array}{l}\text { 4B } \\
\text { Cell }\end{array}$ & $\begin{array}{c}\text { 4B } \\
\text { Detector }\end{array}$ & $\begin{array}{c}\text { 4B } \\
\text { Cell } \\
\text { 6X ESTEP }\end{array}$ & $\begin{array}{c}\text { 4B } \\
\text { Detector } \\
6 \mathrm{X} \text { ESTEP }\end{array}$ & $\begin{array}{c}\text { 4B } \\
\text { Cell } \\
\text { 60X ESTEP }\end{array}$ & $\begin{array}{c}\text { 4B } \\
\text { Detector } \\
60 \mathrm{X} \text { ESTEP }\end{array}$ & Faddegon \\
\hline $\mathbf{0}$ & $2.991 \mathrm{E}+00(1.5)^{*}$ & $3.224 \mathrm{E}+00(0.2)$ & $3.074 \mathrm{E}+00(1.5)$ & $3.287 \mathrm{E}+00(0.2)$ & $2.816 \mathrm{E}+00(1.6)$ & $2.976 \mathrm{E}+00(0.2)$ & $3.42 \mathrm{E}+00(5.0)$ \\
\hline 1 & $2.802 \mathrm{E}+00(0.6)$ & $3.045 \mathrm{E}+00(0.2)$ & $2.837 \mathrm{E}+00(0.5)$ & $3.066 E+00(0.2)$ & $2.617 E+00(0.6)$ & $2.818 E+00(0.2)$ & $3.21 \mathrm{E}+00(5.0)$ \\
\hline 2 & $2.494 \mathrm{E}+00(0.4)$ & $2.670 \mathrm{E}+00(0.2)$ & $2.484 E+00(0.4)$ & $2.672 E+00(0.2)$ & $2.353 \mathrm{E}+00(0.4)$ & $2.498 \mathrm{E}+00(0.2)$ & $2.78 \mathrm{E}+00(5.0)$ \\
\hline 4 & $1.931 E+00(0.3)$ & $2.009 \mathrm{E}+00(0.2)$ & $1.928 \mathrm{E}+00(0.3)$ & $2.015 E+00(0.2)$ & $1.898 \mathrm{E}+00(0.3)$ & $1.974 \mathrm{E}+00(0.2)$ & $2.14 \mathrm{E}+00(5.0)$ \\
\hline 10 & $1.033 \mathrm{E}+00(0.3)$ & $1.043 \mathrm{E}+00(0.2)$ & $1.030 \mathrm{E}+00(0.3)$ & $1.040 \mathrm{E}+00(0.2)$ & $1.049 E+00(0.3)$ & $1.059 \mathrm{E}+00(0.2)$ & $1.06 \mathrm{E}+00(5.0)$ \\
\hline 30 & $2.664 \mathrm{E}-01(0.3)$ & 2.673E-01(0.3) & $2.689 \mathrm{E}-01(0.3)$ & $2.671 \mathrm{E}-01(0.3)$ & $2.694 \mathrm{E}-01(0.3)$ & $2.663 \mathrm{E}-01(0.3)$ & $2.65 \mathrm{E}-01(5.0)$ \\
\hline 60 & $7.284 \mathrm{E}-02(0.5)$ & $7.278 \mathrm{E}-02(0.3)$ & $7.259 \mathrm{E}-02(0.5)$ & $7.269 \mathrm{E}-02(0.3)$ & $7.267 \mathrm{E}-02(0.5)$ & $7.252 \mathrm{E}-02(0.3)$ & $6.66 \mathrm{E}-02(6.0)$ \\
\hline 90 & $3.294 \mathrm{E}-02(0.7)$ & $3.280 \mathrm{E}-02(0.3)$ & $3.301 \mathrm{E}-02(0.7)$ & $3.287 \mathrm{E}-02(0.3)$ & $3.330 \mathrm{E}-02(0.7)$ & $3.291 \mathrm{E}-02(0.3)$ & $2.87 \mathrm{E}-02(6.0)$ \\
\hline
\end{tabular}

$*_{\text {read as }} 2.991 \times 10^{0}$ with $1.5 \%$ error 


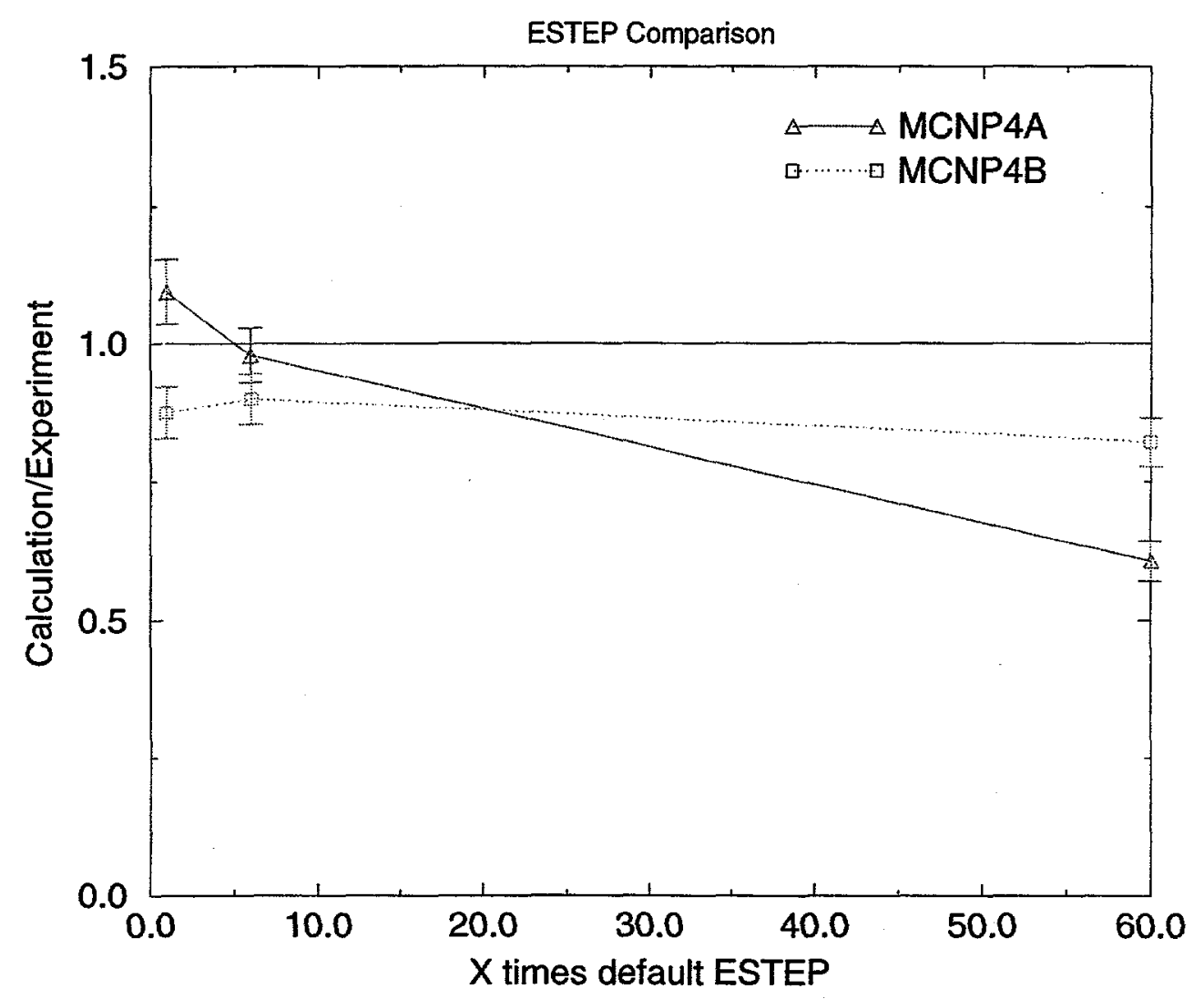

Fig. 23. Ratio of calculation to experiment for a few values of ESTEP; Al target.

\section{Energy Grid Sampling}

The DBCN:18 parameter was varied to examine the differences caused by using either the default "MCNP-style" energy-indexing algorithm (bin-centered treatment), or by using the nearest group boundary method used by ITS3.0. ${ }^{4}$ All precalculated and tabulated data for electrons are stored on an energy grid whose consecutive energy values obey the ratio

$$
\frac{E_{n}}{E_{n-1}}=k
$$

where $k=2^{-1 / 8}$, which results in an average energy loss per major electron step of $8.3 \% .^{1}$

The differences between the bin-centered and nearest bin are illustrated in Fig. 24. For the MCNP style bin-centered energy grid treatment, if an electron has an energy between the grid boundaries $E_{n}$ and $E_{n-1}$, the electron uses the data from group $n-1$. In the nearest bin treatment, if 
the energy of the electron is between the average group energies $\bar{E}_{n}$ and $\bar{E}_{n-1}$, the data from group $n-I$ are used probably in order to account for the fact that the electron is losing energy as it takes a step.

Several comparison studies were done to quantify the effect of the DBCN:18 entry. Tables XVI and XVII give the default and $\mathrm{DBCN}: 18=1$ results for $\mathrm{Al}$ and $\mathrm{Pb}$. These simulations were done using MCNP4B.

Using the nearest group energy treatment has a greater effect for larger angles. For cell tallies, the bremsstrahlung yield drops by only $2.7 \%$ at $0^{\circ}$, which is within the combined statistical uncertainties of the two tallies. At $90^{\circ}$, however, the nearest group method leads to a decrease in the yield by $7.4 \%$. This effect may occur because the scatter distributions at higher angles are more sensitive to changes in energy. Figure 25 shows that emulating ITS gives consistently lower values throughout the spectrum at $90^{\circ}$. At smaller angles, where the integrated yields do not differ significantly, the spectra also agree quite well, as shown in Fig. 26. In both Figs. 25 and 26, only a portion of the bremsstrahlung spectra are shown for clarity.

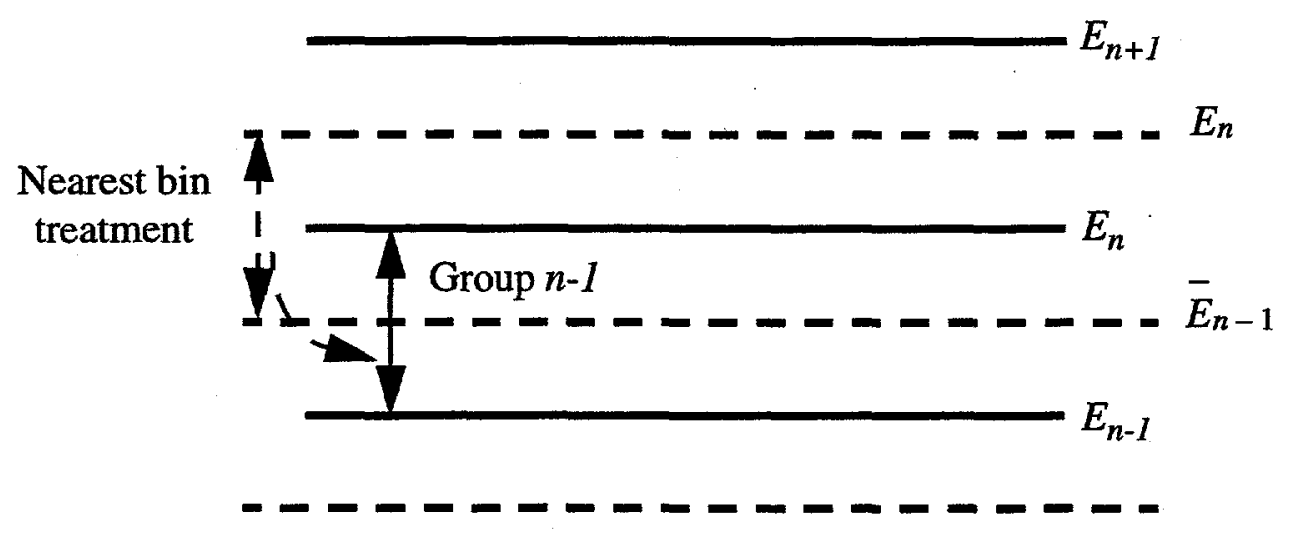

Fig. 24. Schematic for energy group treatment. 
TABLE XVI: DBCN:18 COMPARISONS FOR PB

\begin{tabular}{|c|c|c|c|c|c|}
\hline Angle & $\begin{array}{c}\text { 4B } \\
\text { Cell }\end{array}$ & $\begin{array}{c}\text { 4B } \\
\text { Detector }\end{array}$ & $\begin{array}{c}\text { 4B } \\
\text { Cell } \\
\text { Emulate ITS }\end{array}$ & $\begin{array}{c}\text { 4B } \\
\text { Detector } \\
\text { Emulate ITS }\end{array}$ & Faddegon \\
\hline \hline 0 & $2.640 \mathrm{E}+00(2.0)^{*}$ & $2.797 \mathrm{E}+00(0.3)$ & $2.663 \mathrm{E}+00(2.2)$ & $2.775 \mathrm{E}+00(0.3)$ & $2.92 \mathrm{E}+00(5.0)$ \\
1 & $2.463 \mathrm{E}+00(0.8)$ & $2.667 \mathrm{E}+00(0.3)$ & $2.472 \mathrm{E}+00(0.8)$ & $2.641 \mathrm{E}+00(0.3)$ & $2.80 \mathrm{E}+00(5.0)$ \\
2 & $2.255 \mathrm{E}+00(0.6)$ & $2.380 \mathrm{E}+00(0.3)$ & $2.240 \mathrm{E}+00(0.6)$ & $2.356 \mathrm{E}+00(0.3)$ & $2.48 \mathrm{E}+00(5.0)$ \\
4 & $1.835 \mathrm{E}+00(0.4)$ & $1.883 \mathrm{E}+00(0.3)$ & $1.800 \mathrm{E}+00(0.5)$ & $1.853 \mathrm{E}+00(0.3)$ & $1.99 \mathrm{E}+00(5.0)$ \\
10 & $1.122 \mathrm{E}+00(0.4)$ & $1.125 \mathrm{E}+00(0.3)$ & $1.092 \mathrm{E}+00(0.4)$ & $1.101 \mathrm{E}+00(0.4)$ & $1.2 \mathrm{E}+00(5.0)$ \\
30 & $4.338 \mathrm{E}-01(0.4)$ & $4.310 \mathrm{E}-01(0.5)$ & $4.131 \mathrm{E}-01(0.4)$ & $4.146 \mathrm{E}-01(0.5)$ & $4.47 \mathrm{E}-01(5.0)$ \\
60 & $1.444 \mathrm{E}-01(0.5)$ & $1.434 \mathrm{E}-01(0.5)$ & $1.351 \mathrm{E}-01(0.5)$ & $1.336 \mathrm{E}-01(0.6)$ & $1.29 \mathrm{E}-01(5.0)$ \\
90 & $6.029 \mathrm{E}-02(0.7)$ & $5.860 \mathrm{E}-02(0.7)$ & $5.577 \mathrm{E}-02(0.7)$ & $5.534 \mathrm{E}-02(0.7)$ & $5.19 \mathrm{E}-02(7.0)$ \\
\hline
\end{tabular}

$*_{\text {read as }} 2.640 \times 10^{0}$ with $2.0 \%$ error

TABLE XVII: DBCN:18 COMPARISONS FOR AL

\begin{tabular}{|c|c|c|c|c|c|c|c|}
\hline Angle & $\begin{array}{c}4 \mathrm{~B} \\
\text { Cell }\end{array}$ & $\begin{array}{c}4 \mathrm{~B} \\
\text { Detector }\end{array}$ & $\begin{array}{c}4 \mathrm{~B} \\
\text { Cell } \\
\text { Emulate ITS }\end{array}$ & $\begin{array}{c}4 \mathrm{~B} \\
\text { Detector } \\
\text { Emulate ITS }\end{array}$ & $\begin{array}{c}4 \mathrm{~B} \\
\text { Cell } \\
\text { Emulate ITS } \\
60 \mathrm{X} \text { ESTEP }\end{array}$ & $\begin{array}{c}4 \mathrm{~B} \\
\text { Detector } \\
\text { Emulate ITS } \\
60 \mathrm{X} \text { ESTEP }\end{array}$ & Faddegon \\
\hline \hline 0 & $2.991 \mathrm{E}+00(1.5)^{*}$ & $3.224 \mathrm{E}+00(0.2)$ & $2.909 \mathrm{E}+00(1.6)$ & $3.179 \mathrm{E}+00(0.2)$ & $2.705 \mathrm{E}+00(1.6)$ & $2.929 \mathrm{E}+00(0.2)$ & $3.42 \mathrm{E}+00(5.0)$ \\
1 & $2.802 \mathrm{E}+00(0.6)$ & $3.045 \mathrm{E}+00(0.2)$ & $2.791 \mathrm{E}+00(0.6)$ & $2.994 \mathrm{E}+00(0.2)$ & $2.577 \mathrm{E}+00(0.6)$ & $2.762 \mathrm{E}+00(0.2)$ & $3.21 \mathrm{E}+00(5.0)$ \\
2 & $2.494 \mathrm{E}+00(0.4)$ & $2.670 \mathrm{E}+00(0.2)$ & $2.423 \mathrm{E}+00(0.4)$ & $2.624 \mathrm{E}+00(0.2)$ & $2.299 \mathrm{E}+00(0.4)$ & $2.448 \mathrm{E}+00(0.2)$ & $2.78 \mathrm{E}+00(5.0)$ \\
4 & $1.931 \mathrm{E}+00(0.3)$ & $2.009 \mathrm{E}+00(0.2)$ & $1.889 \mathrm{E}+00(0.3)$ & $1.963 \mathrm{E}+00(0.2)$ & $1.843 \mathrm{E}+00(0.3)$ & $1.918 \mathrm{E}+00(0.2)$ & $2.14 \mathrm{E}+00(5.0)$ \\
10 & $1.033 \mathrm{E}+00(0.3)$ & $1.043 \mathrm{E}+00(0.2)$ & $9.924 \mathrm{E}-01(0.3)$ & $1.001 \mathrm{E}+00(0.2)$ & $1.009 \mathrm{E}+00(0.3)$ & $1.016 \mathrm{E}+00(0.2)$ & $1.06 \mathrm{E}+00(5.0)$ \\
30 & $2.664 \mathrm{E}-01(0.3)$ & $2.673 \mathrm{E}-01(0.3)$ & $2.505 \mathrm{E}-01(0.4)$ & $2.498 \mathrm{E}-01(0.3)$ & $2.504 \mathrm{E}-01(0.4)$ & $2.495 \mathrm{E}-01(0.3)$ & $2.65 \mathrm{E}-01(5.0)$ \\
60 & $7.284 \mathrm{E}-02(0.5)$ & $7.278 \mathrm{E}-02(0.3)$ & $6.733 \mathrm{E}-02(0.5)$ & $6.697 \mathrm{E}-02(0.3)$ & $6.687 \mathrm{E}-02(0.5)$ & $6.670 \mathrm{E}-02(0.3)$ & $6.66 \mathrm{E}-02(6.0)$ \\
90 & $3.294 \mathrm{E}-02(0.7)$ & $3.280 \mathrm{E}-02(0.3)$ & $3.050 \mathrm{E}-02(0.7)$ & $3.020 \mathrm{E}-02(0.3)$ & $3.055 \mathrm{E}-02(0.7)$ & $3.032 \mathrm{E}-02(0.3)$ & $2.87 \mathrm{E}-02(6.0)$ \\
\hline
\end{tabular}

$*_{\text {read }} 2.991 \times 10^{0}$ with $1.5 \%$ error

Table XVII also includes data from a simulation combining the effect of ESTEP set to 60 times the default and DBCN:18=1. Increasing ESTEP affects the $\mathrm{DBCN}: 18=1$ results in the same way that it does the default MCNP simulation, i.e., the two parameters act independently and do not counteract each other. The forward angles exhibit the largest effect, with a $7 \%$ decrease in the $0^{\circ}$ cell tally, and practically no difference at $90^{\circ}$. 


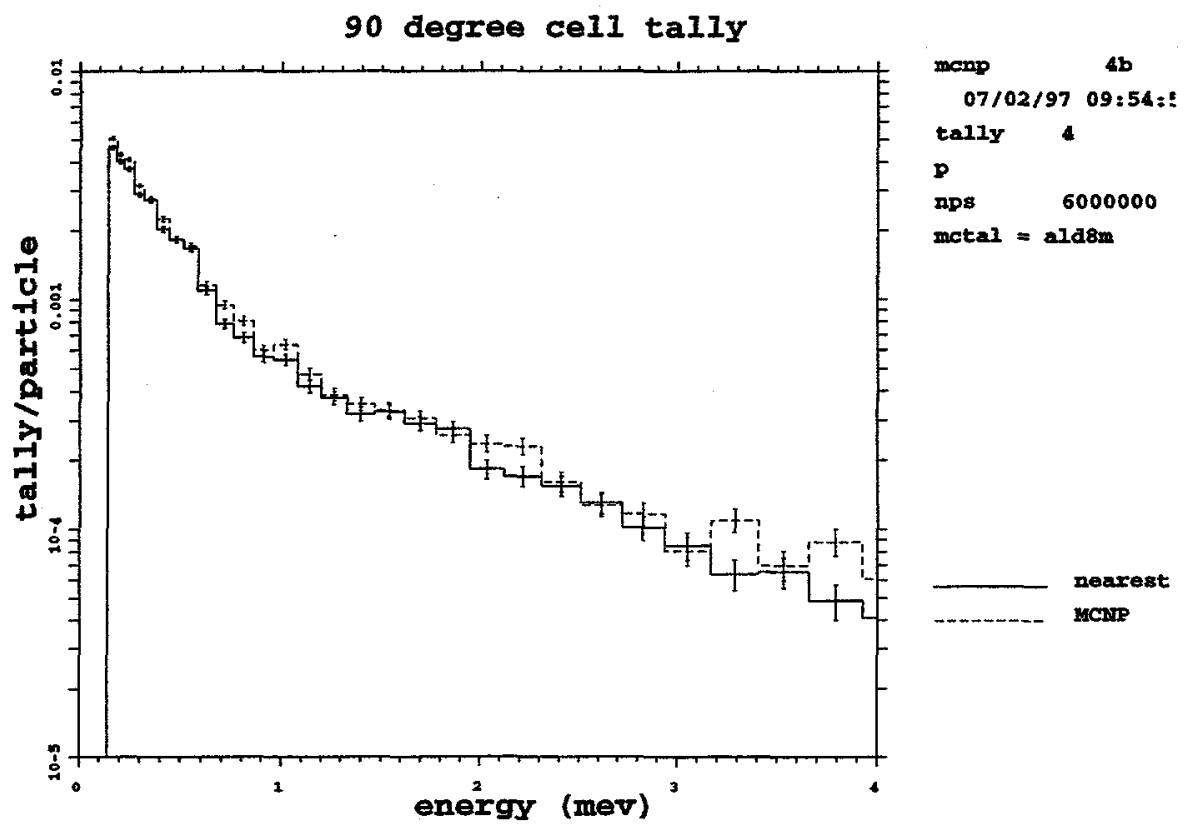

Fig. 25. Bremsstrahlung spectrum at $90^{\circ}$ for default MCNP and DBCN18=1 (nearest bin).

The impact of the energy grid treatment was also studied for the electron transmission and reflection calculations. The comparisons for the default simulations were presented in Section III.A.3. Transmission coefficients for $10.2 \mathrm{MeV}$ electrons incident on $\mathrm{C}, \mathrm{Ag}$, and $\mathrm{U}$ foils of varying thicknesses, including the DBCN:18 simulations, are presented in Figs. 27-29. The DBCN:18 results are consistently lower than the default results. Using the nearest bin treatment most likely results in more electron scattering and energy loss, and therefore fewer electrons are able to escape the slab. The differences between the two simulations for carbon are more pronounced than for either silver or uranium, probably because carbon has a much lower $\mathrm{Z}$ than either silver or uranium.

For silver, The $\mathrm{DBCN}: 18$ simulation and experiment generally agree to within 5\%about a 5\% improvement over default MCNP. For carbon, the agreement with experiment when using the nearest bin treatment is much improved, especially for thicker foils. The nearest bin treatment gives agreement with experiment that is usually within about $8 \%$, with the exception of the last few points. For uranium, default MCNP agrees with experiment a few percent better than when setting DBCN:18=1. 


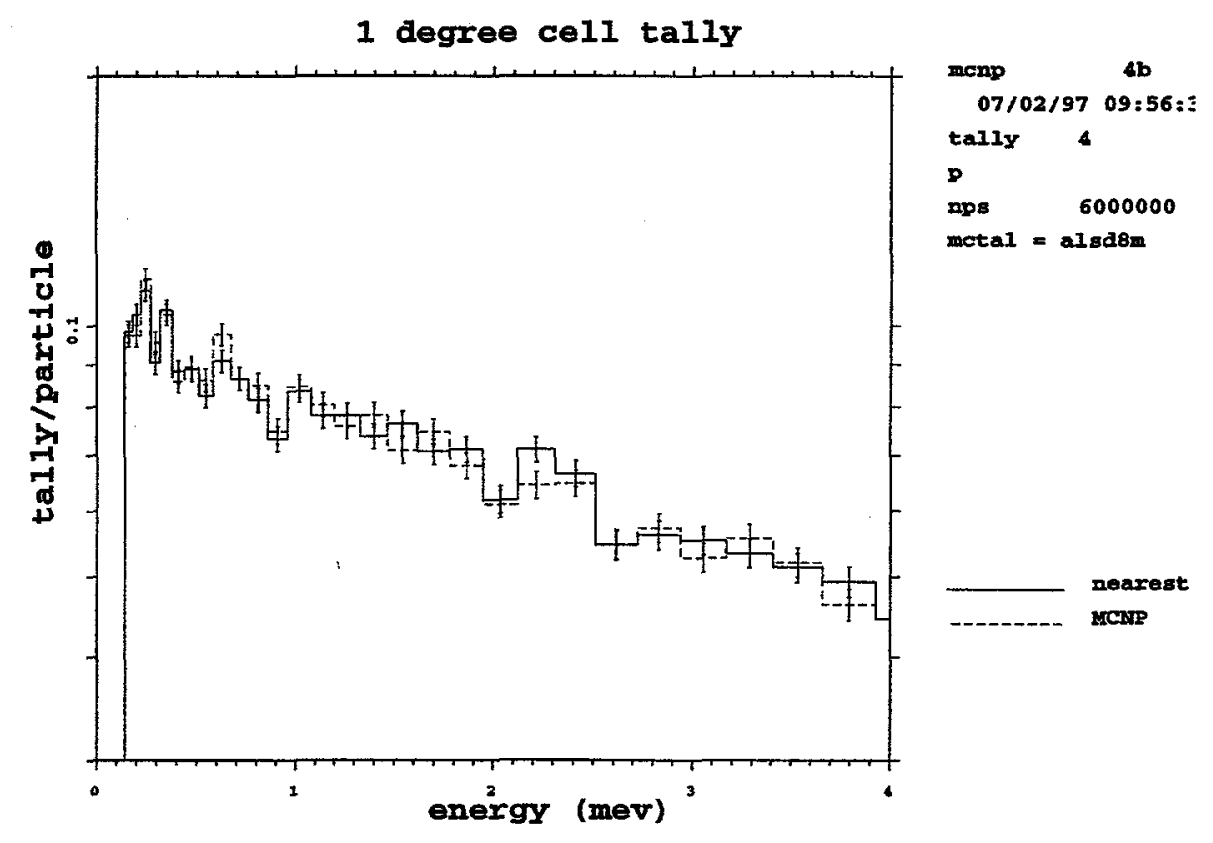

Fig. 26. Bremsstrahlung spectrum at $1^{\circ}$ for default MCNP and DBCN18=1 (nearest bin).

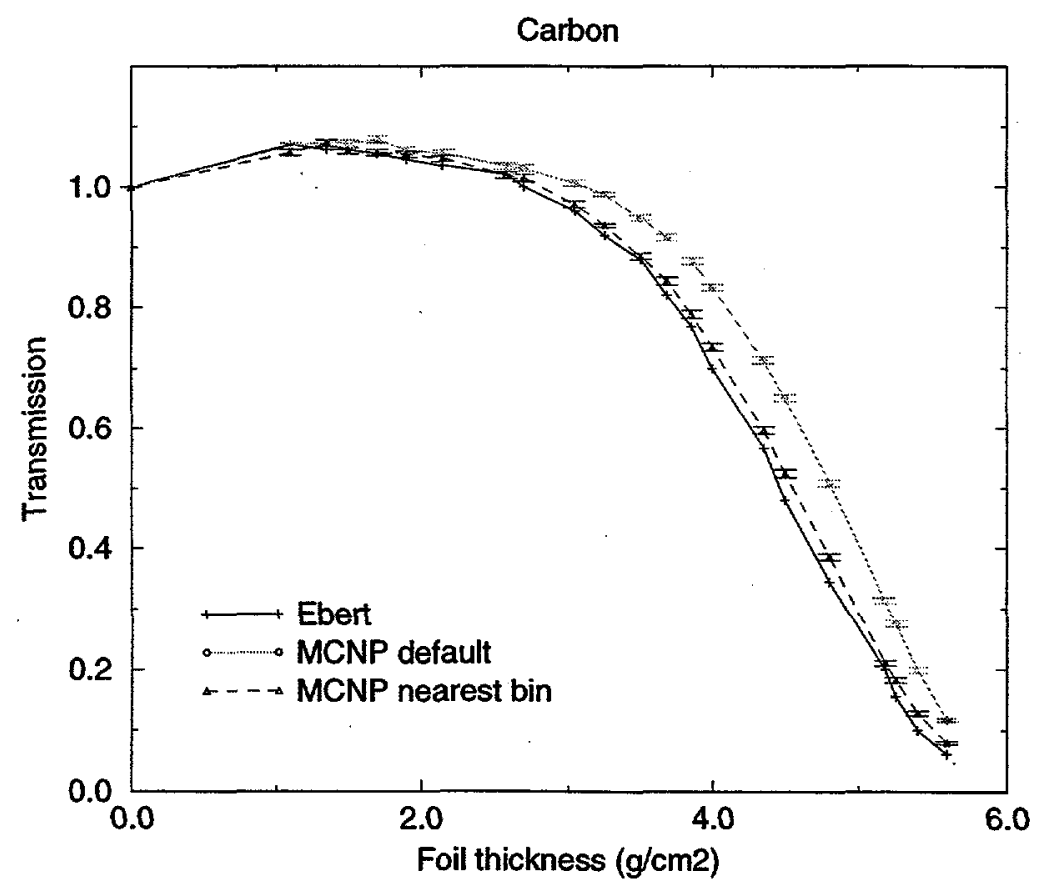

Fig. 27. Comparison of transmission coefficients for $10.2 \mathrm{MeV}$ electrons incident on $\mathrm{C}$ foils. 


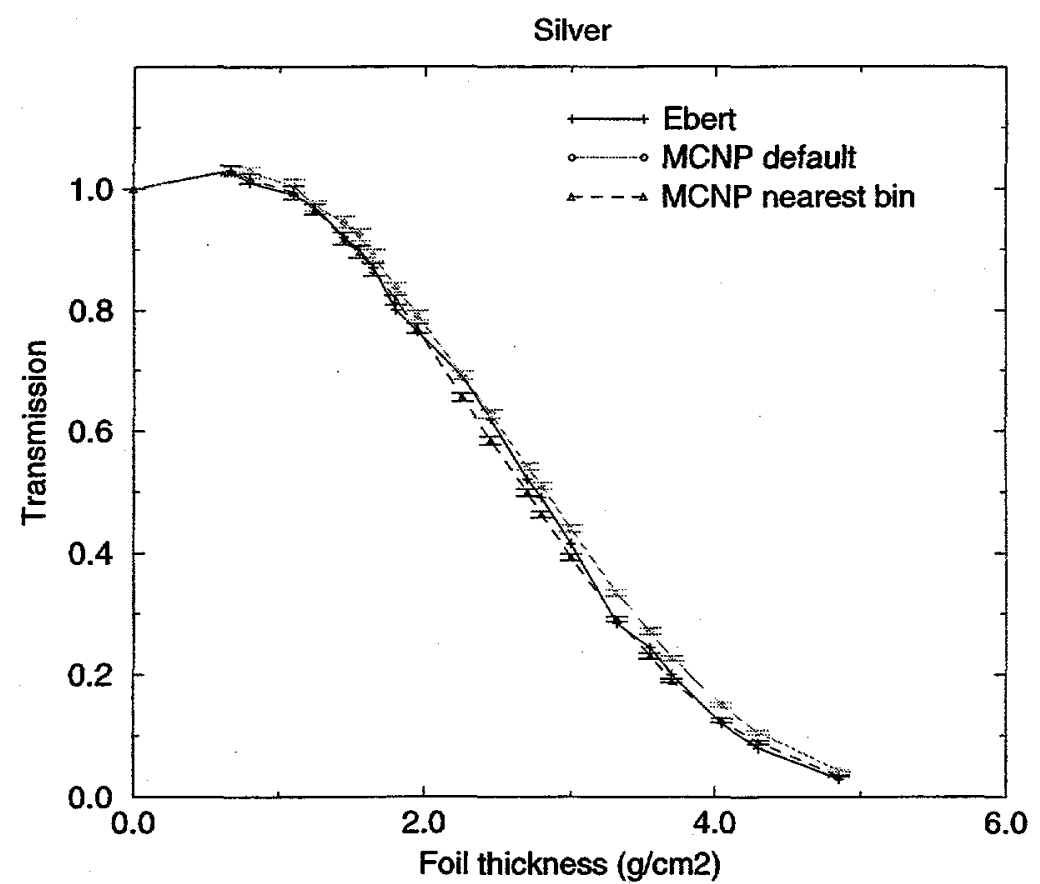

Fig. 28. Comparison of transmission coefficients for $10.2 \mathrm{MeV}$ electrons incident on $\mathrm{Ag}$ foils.

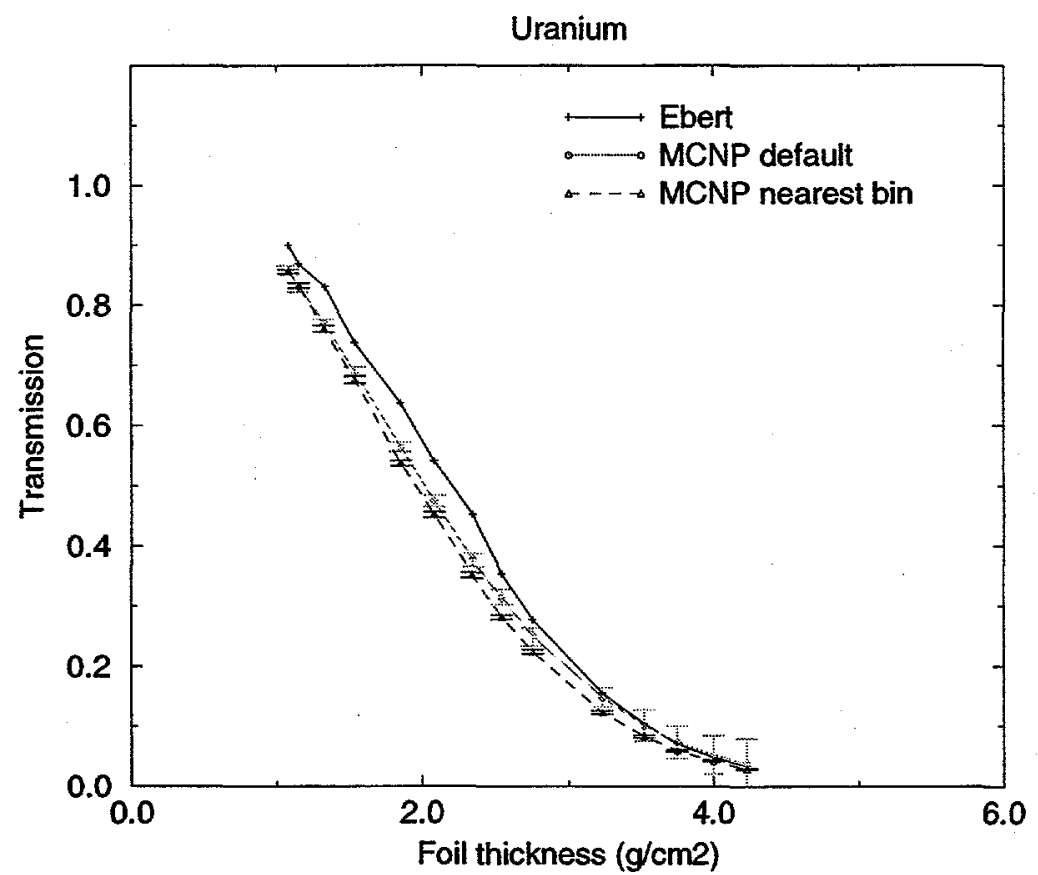

Fig. 29. Comparison of transmission coefficients for $10.2 \mathrm{MeV}$ electrons incident on $\mathrm{U}$ foils. 
Table XVIII shows the results of the backscatter benchmark calculations, including the DBCN:18=1 results. The values in parentheses are the percent errors. The two simulations for each material agreed to within $10 \%$ of each other. The $\mathrm{DBCN}: 18=1$ simulation results were consistently lower than the default MCNP simulations, again because of the difference in energy grid sampling which causes fewer electrons to be able to escape the slab.

The simulations using the nearest bin method agree with experiment to a greater degree than the transmission measurements. The agreement is $1-10 \%$, which is within the combined statistical uncertainties of simulation and experiment, with the exception of the outlying point for carbon at $10.2 \mathrm{MeV}$.

TABLE XVIII: ELECTRON SATURATION BACKSCATTER COMPARISON

\begin{tabular}{|c|c|c|c|c|c|c|}
\hline Material & $\begin{array}{c}\text { Electron } \\
\text { Energy } \\
(\mathrm{MeV})\end{array}$ & Ebert & Dressel & Tabata & MCNP & $\begin{array}{c}\text { MCNP } \\
\text { DBCN:18=1 }\end{array}$ \\
\hline \hline $\mathrm{C}$ & & $4.0 \mathrm{E}-03$ & $9.0 \mathrm{E}-03$ & $3.20 \mathrm{E}-03$ & $5.846 \mathrm{E}-03(4.0)$ & $5.175 \mathrm{E}-03(4.0)^{*}$ \\
$\mathrm{Ag}$ & 10.2 & $7.4 \mathrm{E}-02$ & $1.8 \mathrm{E}-01$ & $7.35 \mathrm{E}-02$ & $8.465 \mathrm{E}-02(3.0)$ & $7.675 \mathrm{E}-02(3.0)$ \\
& & $1.47 \mathrm{E}-01$ & $3.3 \mathrm{E}-01$ & $1.36 \mathrm{E}-01$ & $1.780 \mathrm{E}-01(2.0)$ & $1.569 \mathrm{E}-01(2.0)$ \\
\hline $\mathrm{C}$ & & $5.0 \mathrm{E}-03$ & $8.6 \mathrm{E}-03$ & $4.00 \mathrm{E}-03$ & $6.122 \mathrm{E}-03(4.0)$ & $5.547 \mathrm{E}-03(4.0)$ \\
$\mathrm{Ag}$ & 8.0 & $9.5 \mathrm{E}-02$ & $2.0 \mathrm{E}-01$ & $9.70 \mathrm{E}-02$ & $1.149 \mathrm{E}-01(2.0)$ & $1.034 \mathrm{E}-01(3.0)$ \\
$\mathrm{U}$ & & $1.95 \mathrm{E}-01$ & $3.8 \mathrm{E}-01$ & $1.72 \mathrm{E}-01$ & $2.179 \mathrm{E}-01(2.0)$ & $1.961 \mathrm{E}-01(2.0)$ \\
\hline $\mathrm{C}$ & & $6.0 \mathrm{E}-03$ & $1.0 \mathrm{E}-02$ & $5.00 \mathrm{E}-03$ & $6.856 \mathrm{E}-03(3.0)$ & $6.542 \mathrm{E}-03(4.0)$ \\
$\mathrm{Ag}$ & 6.0 & $1.39 \mathrm{E}-01$ & $2.4 \mathrm{E}-01$ & $1.29 \mathrm{E}-02$ & $1.504 \mathrm{E}-01(2.0)$ & $1.334 \mathrm{E}-01(2.0)$ \\
$\mathrm{U}$ & & $2.45 \mathrm{E}-01$ & $4.5 \mathrm{E}-01$ & $2.28 \mathrm{E}-01$ & $2.780 \mathrm{E}-01(1.01)$ & $2.581 \mathrm{E}-01(1.0)$ \\
\hline
\end{tabular}

$*_{\text {read as }} 5.175 \times 10^{-3}$ with $4.0 \%$ error

\section{E. Splitting Schemes for Bremsstrahlung Production}

The effect of bremsstrahlung sampling using the PHYS:E BNUM biasing parameter was explored. BNUM is a variance reduction tool that specifies the production of BNUM times the analog number of bremsstrahlung photons, each with weight 1/BNUM. Simulations with and without BNUM were compared to verify the consistency of the results and to quantify improvements in the tally Figure-of-Merit (FOM). The FOM, as calculated by MCNP, is given by

$$
F O M=\frac{1}{R^{2} T}
$$


where $R$ is the relative error, and $T$ is the computer time used in the MCNP problem. The FOM can be better understood if it is written in the following manner:

$$
T=\frac{1}{R^{2} F O M}
$$

This relation enables one to find the computer time needed to reach a desired value of $R$. The higher the FOM, the less computer time will be needed to reach the desired tally error. An alternate version of MCNP4B, using a special BNUM patch, was also included in the comparison. This patch (see Appendix A) alters the BNUM biasing from producing BNUM identical bremsstrahlung photons to sampling a different bremsstrahlung photon BNUM times.

The standard BNUM splitting scheme was verified by comparing simulations of the Starfelt and Koch experiment, described in Section II.C. Figures 30 and 31 show the results using default settings (BNUM=1) and BNUM equal to 20 for $0^{\circ}$ and $12^{\circ}$ cell tallies, respectively. All of the BNUM runs were done with ENUM set to 1/BNUM. The ENUM parameter controls how many photon-induced secondary electrons are produced. This production is done to keep the number of electrons in the problem the same as in an analog calculation, thus avoiding excessive computer times. For both angles, the agreement between the two simulations is within statistical uncertainty.

The effect of the variance reduction parameter BNUM was also explored in further detail for the Faddegon experiment described in Section II.A. First, the behavior of BNUM was verified in that it reduced the tally errors while still giving an answer consistent with an analog calculation (BNUM=1). Table XIX shows these results for default MCNP, MCNP with BNUM=20, and MCNP with the modified sampling scheme for BNUM. Again, the BNUM runs were done with ENUM set to 1/BNUM. Also, these simulations were all done for the same number of electron histories.

The standard versions of MCNP give nearly identical results independent of the value BNUM. The patched version does not track the standard version exactly, but the results are generally within $2-3 \%$. The decrease in error is also evident from these data, most notably for the $0^{\circ}$ tally. Standard MCNP BNUM sampling reduces the error by a factor of about 1.3 , whereas the modified BNUM gives an improvement of a factor of 4.2. 
o degree

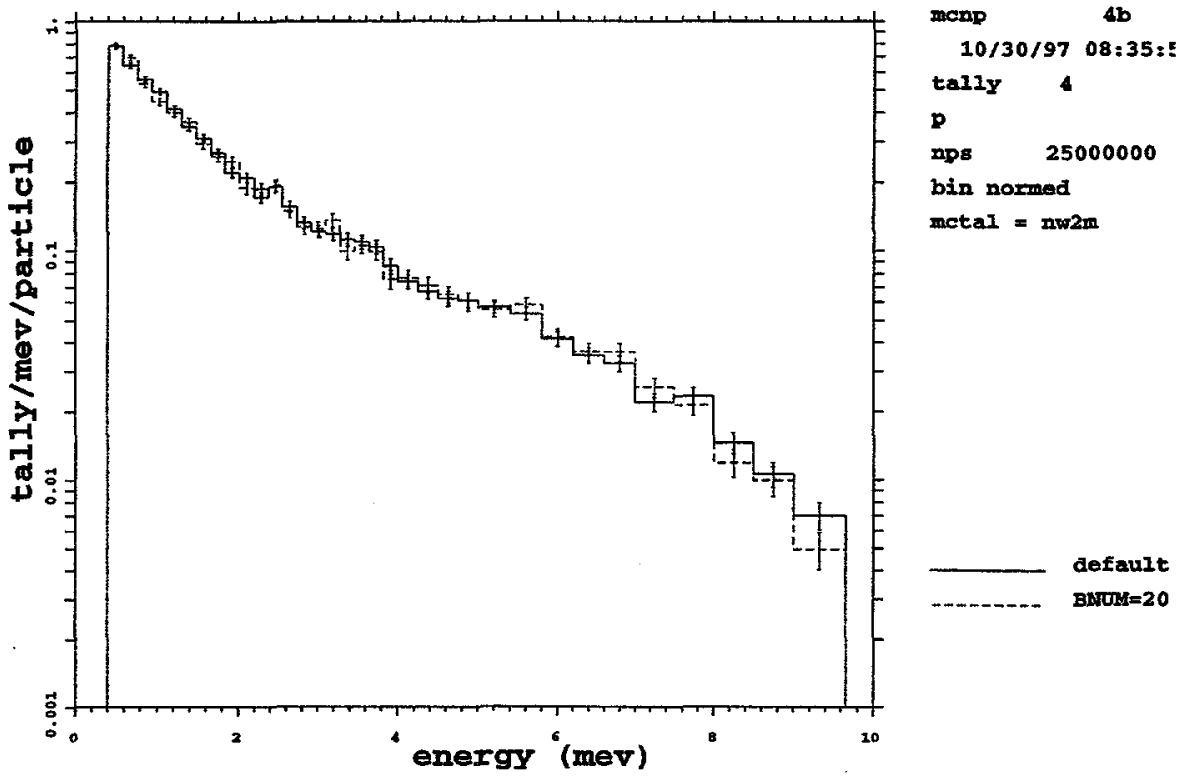

Fig. 30. A comparison of the Starfelt and Koch bremsstrahlung spectra for default $(B N U M=1)$ and standard $B N U M=20$ variance reduction for the $0^{\circ}$ cell tally.

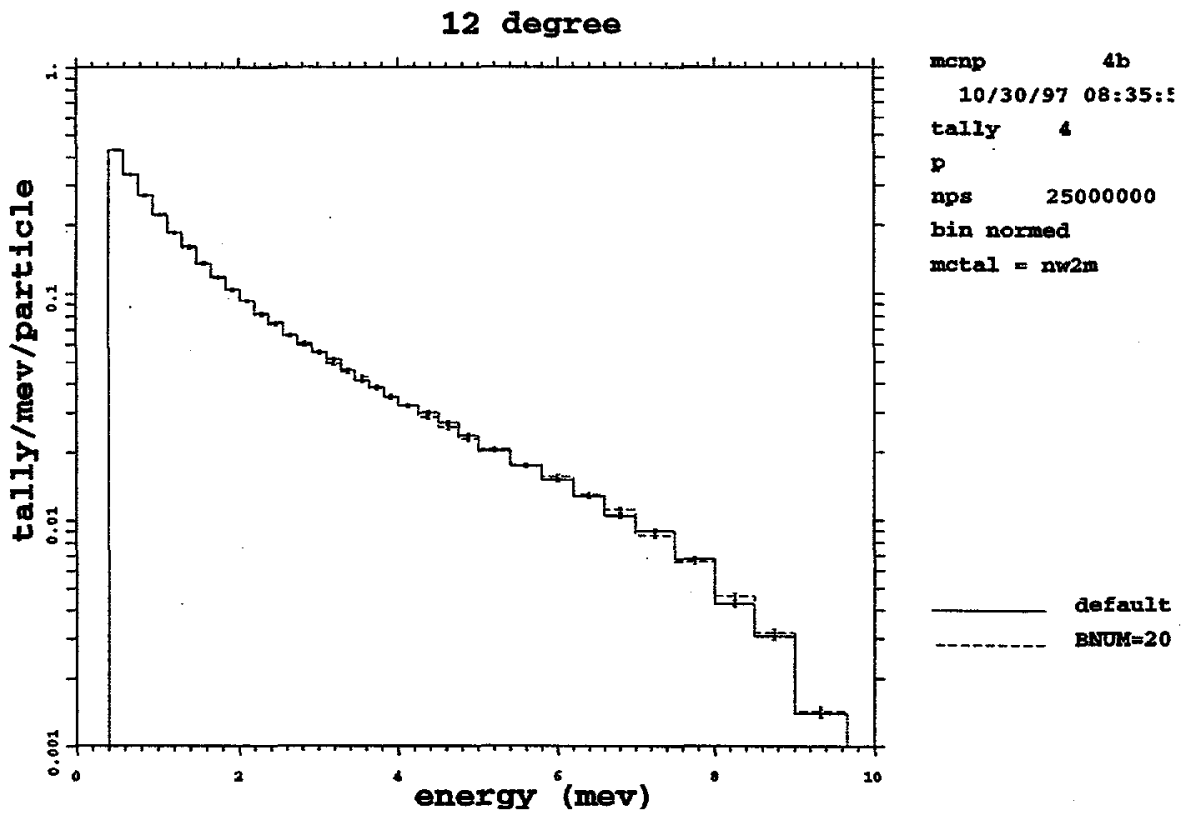

Fig. 31. A comparison of the Starfelt and Koch bremsstrahlung spectra for default (BNUM=1) and standard BNUM=20 variance reduction for the $12^{\circ}$ cell tally. 
TABLE XIX: BNUM COMPARISONS FOR PB

\begin{tabular}{|c|c|c|c|c|c|c|}
\hline Angle & $\begin{array}{l}\text { 4B } \\
\text { Cell }\end{array}$ & $\begin{array}{c}\text { 4B } \\
\text { Detector }\end{array}$ & $\begin{array}{c}\text { 4B } \\
\text { Cell } \\
\text { BNUM=20 } \\
\text { ENUM=1/20 }\end{array}$ & $\begin{array}{c}4 \mathrm{~B} \\
\text { Detector } \\
\text { BNUM=20 } \\
\text { ENUM }=1 / 20\end{array}$ & $\begin{array}{c}\text { 4B patch } \\
\text { Cell } \\
\text { BNUM=20 } \\
\text { ENUM }=1 / 20\end{array}$ & $\begin{array}{c}\text { 4B patch } \\
\text { Detector } \\
\text { BNUM=20 } \\
\text { ENUM }=1 / 20\end{array}$ \\
\hline 0 & $2.638 \mathrm{E}+00(2.1)^{*}$ & $2.797 \mathrm{E}+00(0.3)$ & $2.656 \mathrm{E}+00(1.6)$ & $2.798 \mathrm{E}+00(0.3)$ & $2.593 \mathrm{E}+00(0.5)$ & $2.768 \mathrm{E}+00(0.1)$ \\
\hline 1 & $2.462 E+00(0.8)$ & $2.667 \mathrm{E}+00(0.3)$ & $2.481 \mathrm{E}+00(0.6)$ & $2.664 \mathrm{E}+00(0.3)$ & $2.450 \mathrm{E}+00(0.2)$ & $2.628 \mathrm{E}+00(0.1)$ \\
\hline 2 & $2.255 \mathrm{E}+00(0.6)$ & $2.380 \mathrm{E}+00(0.3)$ & $2.268 E+00(0.4)$ & $2.372 E+00(0.3)$ & $2.229 \mathrm{E}+00(0.2)$ & $2.350 \mathrm{E}+00(0.1)$ \\
\hline 4 & $1.835 \mathrm{E}+00(0.4)$ & $1.883 \mathrm{E}+00(0.3)$ & $1.833 \mathrm{E}+00(0.3)$ & $1.891 \mathrm{E}+00(0.3)$ & $1.796 \mathrm{E}+00(0.1)$ & $1.854 \mathrm{E}+00(0.09)$ \\
\hline 10 & $1.122 \mathrm{E}+00(0.4)$ & $1.125 \mathrm{E}+00(0.3)$ & $1.124 \mathrm{E}+00(0.3)$ & $1.128 \mathrm{E}+00(0.3)$ & $1.100 \mathrm{E}+00(0.1)$ & $1.104 \mathrm{E}+00(0.09)$ \\
\hline 30 & $4.347 \mathrm{E}-01(0.3)$ & $4.316 \mathrm{E}-01(0.4)$ & $4.337 \mathrm{E}-01(0.3)$ & $4.299 \mathrm{E}-01(0.4)$ & $4.170 \mathrm{E}-01(0.1)$ & $4.162 \mathrm{E}-01(0.1)$ \\
\hline 60 & $1.449 \mathrm{E}-01(0.4)$ & $1.439 \mathrm{E}-01(0.5)$ & $1.445 \mathrm{E}-01(0.3)$ & $1.423 \mathrm{E}-01(0.5)$ & $1.374 \mathrm{E}-01(0.2)$ & $1.363 \mathrm{E}-01(0.1)$ \\
\hline 90 & $6.017 \mathrm{E}-02(0.6)$ & $5.875 \mathrm{E}-02(0.6)$ & $5.986 \mathrm{E}-02(0.4)$ & $5.946 \mathrm{E}-02(0.6)$ & $5.850 \mathrm{E}-02(0.2)$ & $5.785 \mathrm{E}-02(0.1)$ \\
\hline
\end{tabular}

*read as $2.638 \times 10^{0}$ with $2.1 \%$ error

Figures 32 and 33 show the effect on the spectral data when using BNUM variance reduction for the $0^{\circ}$ cell tally. From both figures it is evident that using BNUM does not significantly alter the tally data. The spectral statistics are greatly improved using the modified BNUM sampling, as shown in Fig. 33. The improvement in the spectral statistics is not as great when using the standard BNUM sampling. Figures 34 and 35 show the spectral data for the $2^{\circ}$ cell tally. This tally converges much faster than the $0^{\circ}$ cell tally, so the improvement in statistics is not as pronounced.

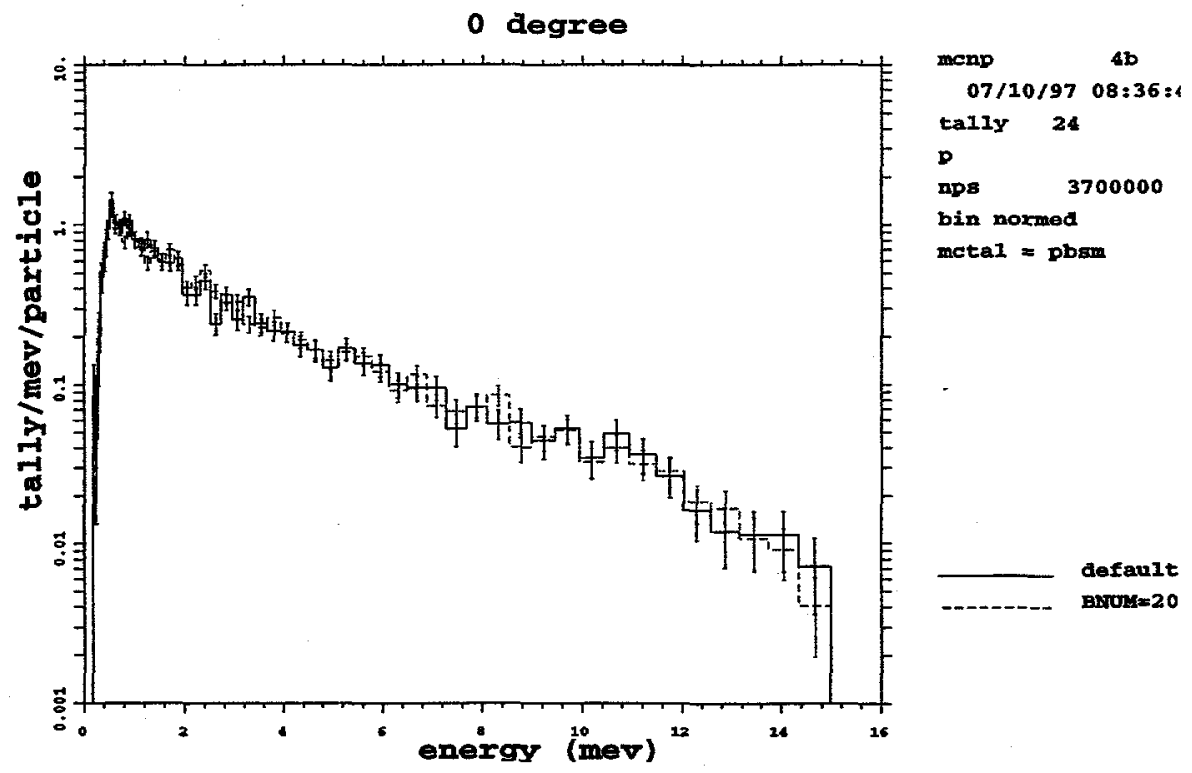

Fig. 32. A comparison of the $\mathrm{Pb}$ bremsstrahlung spectra for default (BNUM=1) and standard $B N U M=20$ variance reduction for the $0^{\circ}$ cell tally. 
O degree

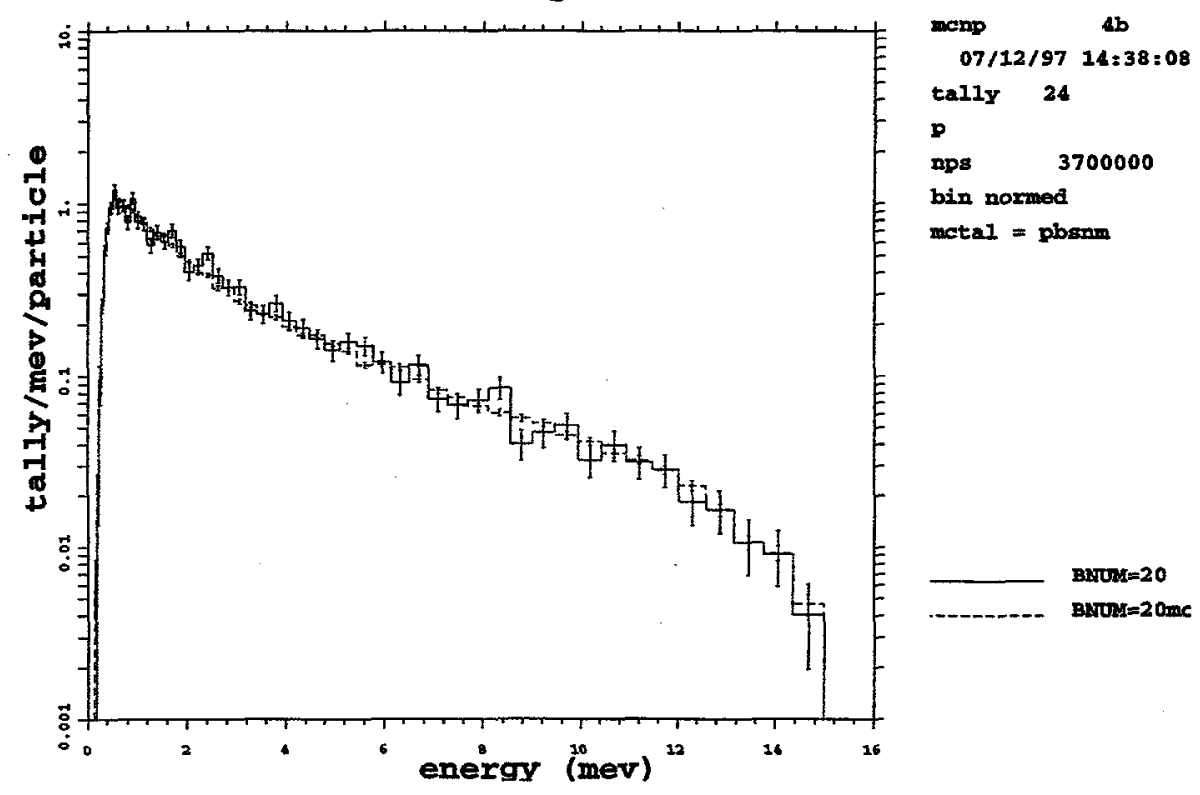

Fig. 33. A comparison of the $\mathrm{Pb}$ bremsstrahlung spectra for standard and modified BNUM sampling $(B N U M=20)$ variance reduction for the $0^{\circ}$ cell tally.

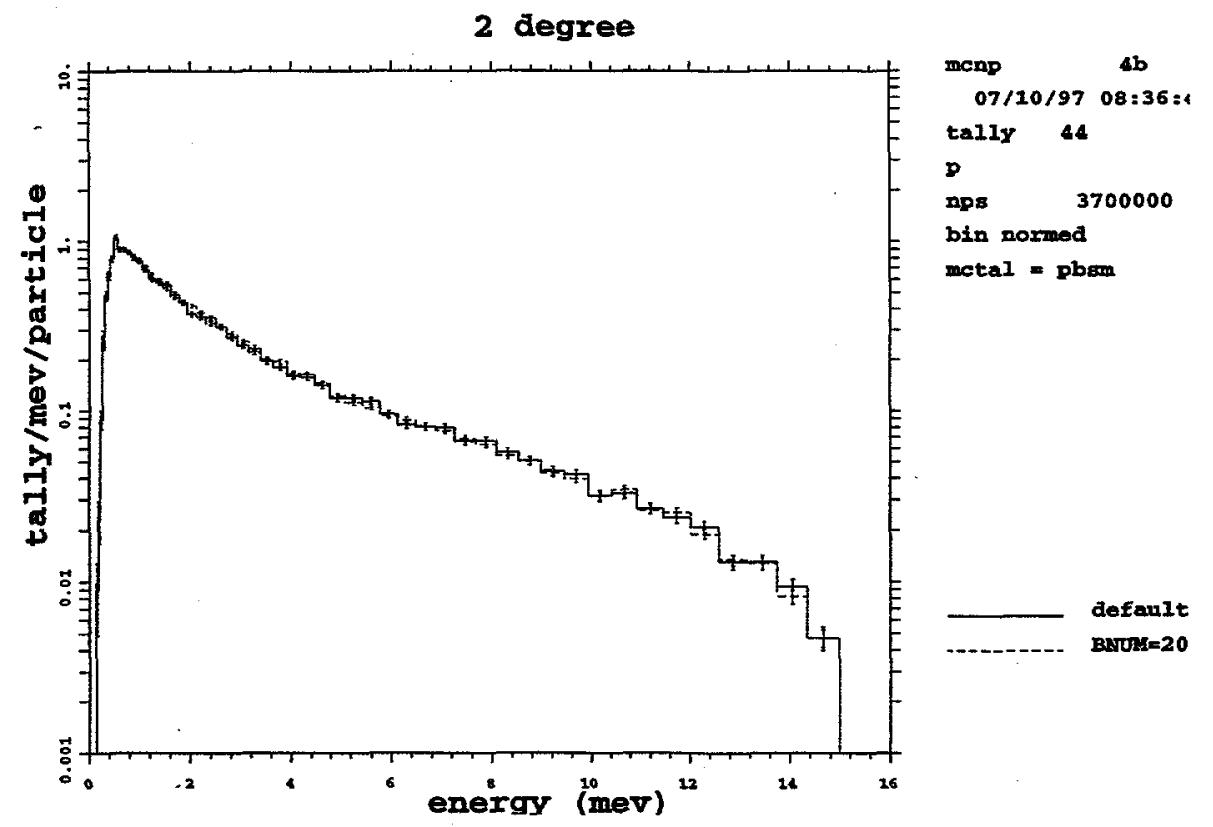

Fig. 34. A comparison of the Pb bremsstrahlung spectra for default (BNUM=1) and standard BNUM $=20$ variance reduction for the $2^{\circ}$ cell tally. 


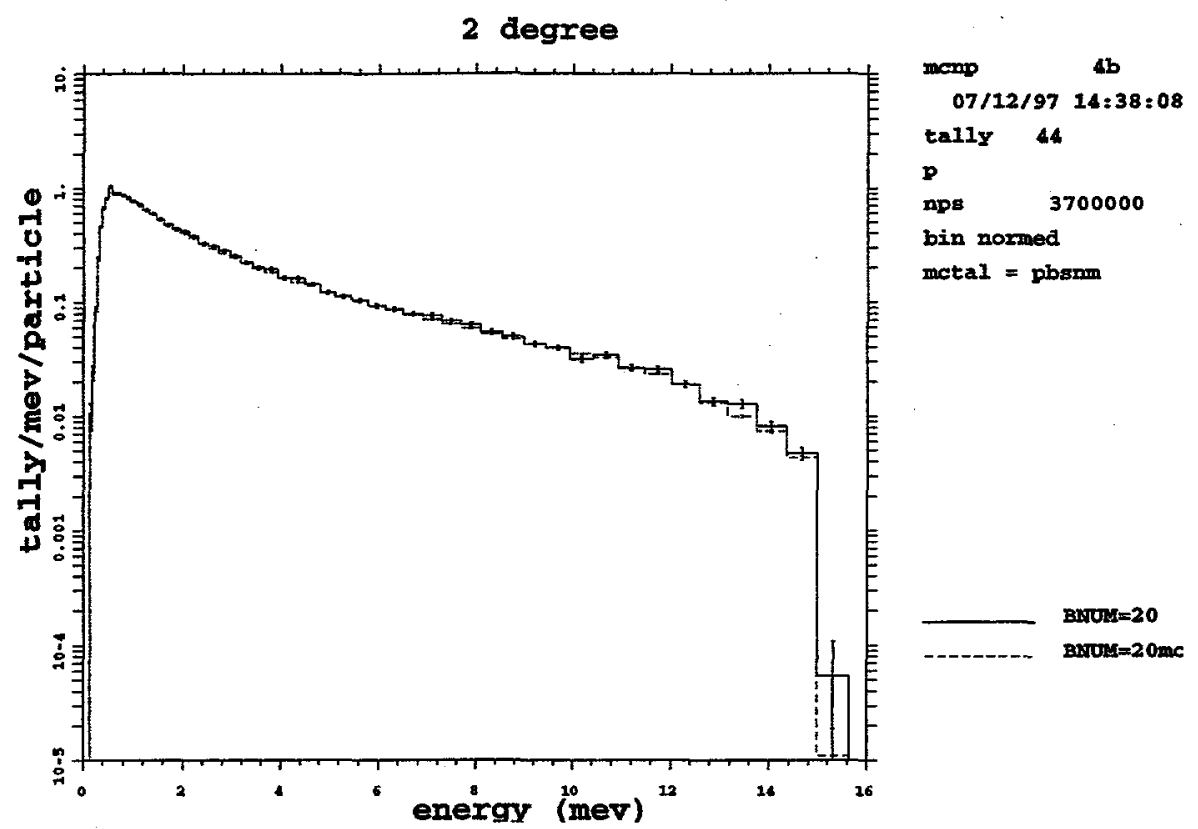

Fig. 35. A comparison of the Pb bremsstrahlung spectra for standard and modified BNUM sampling (BNUM=20) variance reduction for the $2^{\circ}$ cell tally.

A detailed study of the tally Figures-of-Merit (FOM) was done for the standard and modified BNUM sampling schemes. Simulations were done for BNUM values of $1,3,10,20,30$, and 100. It is speculated that the following trends would be less clear if a thicker target were used and the photons underwent more interactions in the target. Figure 36 shows the ratio of the FOM for the modified BNUM sampling scheme to the FOM for the standard scheme. The trends are similar for each angle, although higher gains in the FOM are possible for smaller angles. The FOM is much greater for the patched version of MCNP than for the standard MCNP because the code is sampling over a wide range of angles, rather than at one particular angle. This sampling increases the tally efficiency for the cell tallies, which cover a very small angular range. The ratio of the FOM begins to drop off as BNUM increases, essentially the result of the tally having already converged, with the additional photons created for higher values of BNUM resulting only in an increase in computer time. Figure 37 shows ratio of FOM for the detector tallies. The ratio of FOM for a particular BNUM is inversely proportional to the magnitude of the tally angle. The FOM for detector tallies (see Fig. 37) behaves similarly to the cell tallies. The FOM once again levels off for high values of BNUM. Again, this leveling results from the fact that the tally has 
already converged, and additional photons slow down the calculation. Detector tallies tend to converge faster than cell tallies because they are deterministic estimates of flux, rather than the result of actual particle transport to the detector region. Every particle contributes to a detector tally, regardless of splitting in the default code. Thus, the detector response to BNUM is opposite to that of the cell tallies in that the ratio of FOM for a particular BNUM is proportional to the tally angle.

Figures 38-41 give the ratio of the FOM for the particular value of BNUM $\left(\mathrm{FOM}_{\mathbf{i}}\right)$ to the FOM for the analog case $\left(\mathrm{FOM}_{1}\right)$, for either the standard or modified BNUM sampling scheme. For both cell and detector tallies, the modified BNUM sampling always produces a FOM greater than the analog case. For the standard BNUM sampling and detector tallying, however, increasing BNUM usually results in a degradation in the FOM. For standard BNUM sampling, using BNUM should not lead to gains in the FOM because only one photon contributes to the detector tally, regardless of BNUM. Figure 40 reinforces this fact in that the FOM decreases as BNUM is increased, since more computer time is being spent tracking the extra photons. A degradation in performance is also the case for standard sampling and cell tallies for BNUM greater than 20.

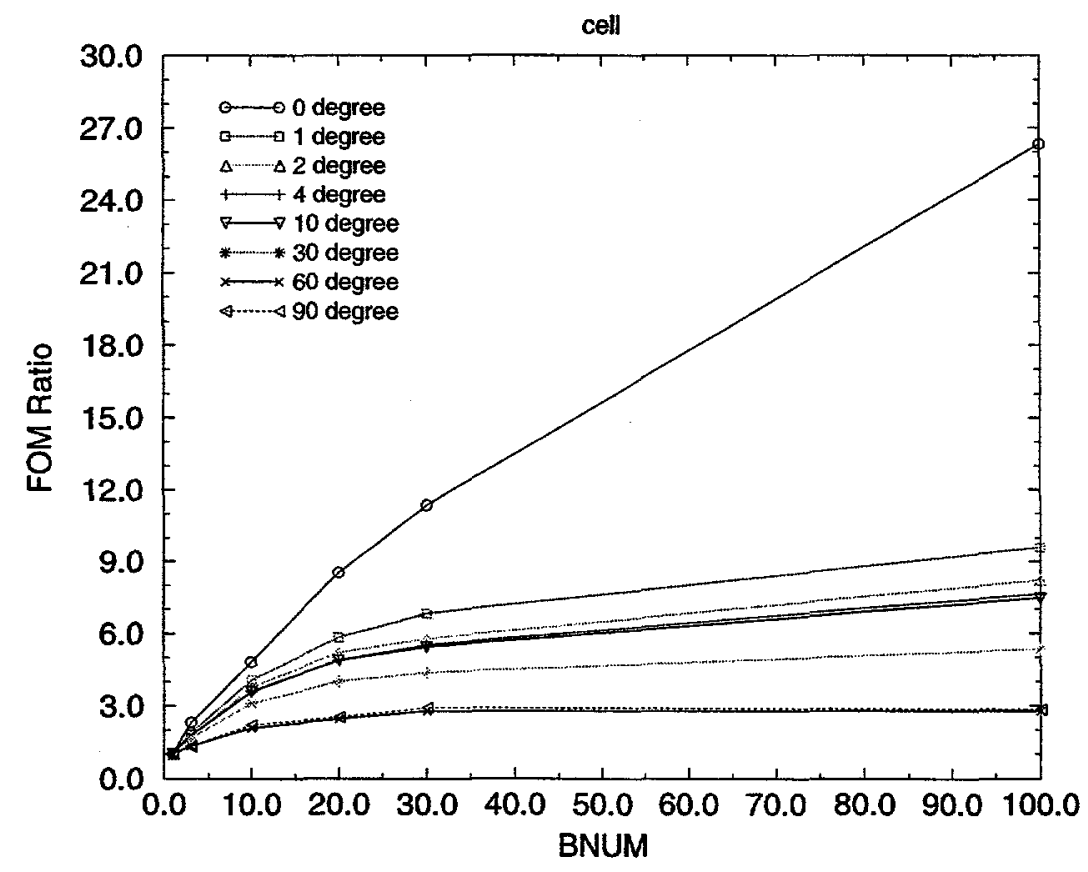

Fig. 36. Ratio of modified FOM to standard FOM vs BNUM for photon flux cell tallies. 
Figures 42 and 43 show the actual FOM values for the photon current and flux for the downstream face of the target. Since these are integrated quantities over a large angular range sampling, more photons will not greatly improve the FOM. The FOM do change with BNUM because different numbers of photons are escaping out the sides of the target and/or being absorbed in the target.

\section{F. $20 \mathrm{MeV}$ Electrons in Water}

This section of the report describes a suite of electron depth dose calculations done with MCNP4xq, a preliminary version of MCNP4B. Energy deposition is one of the most widely recognized benchmarks for electron calculations. These calculations augment the growing database of electron/photon benchmark calculations.

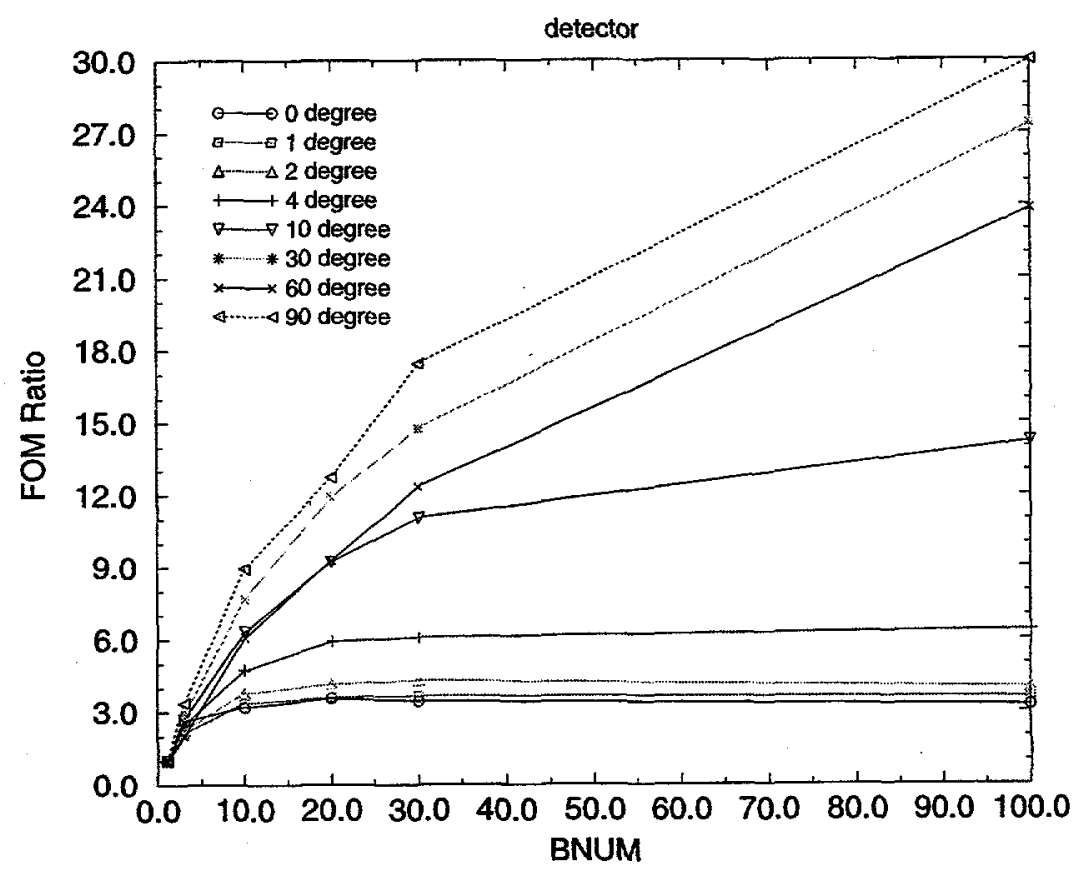

Fig. 37. Ratio of modified FOM to standard FOM vs BNUM for photon flux detector tallies. 


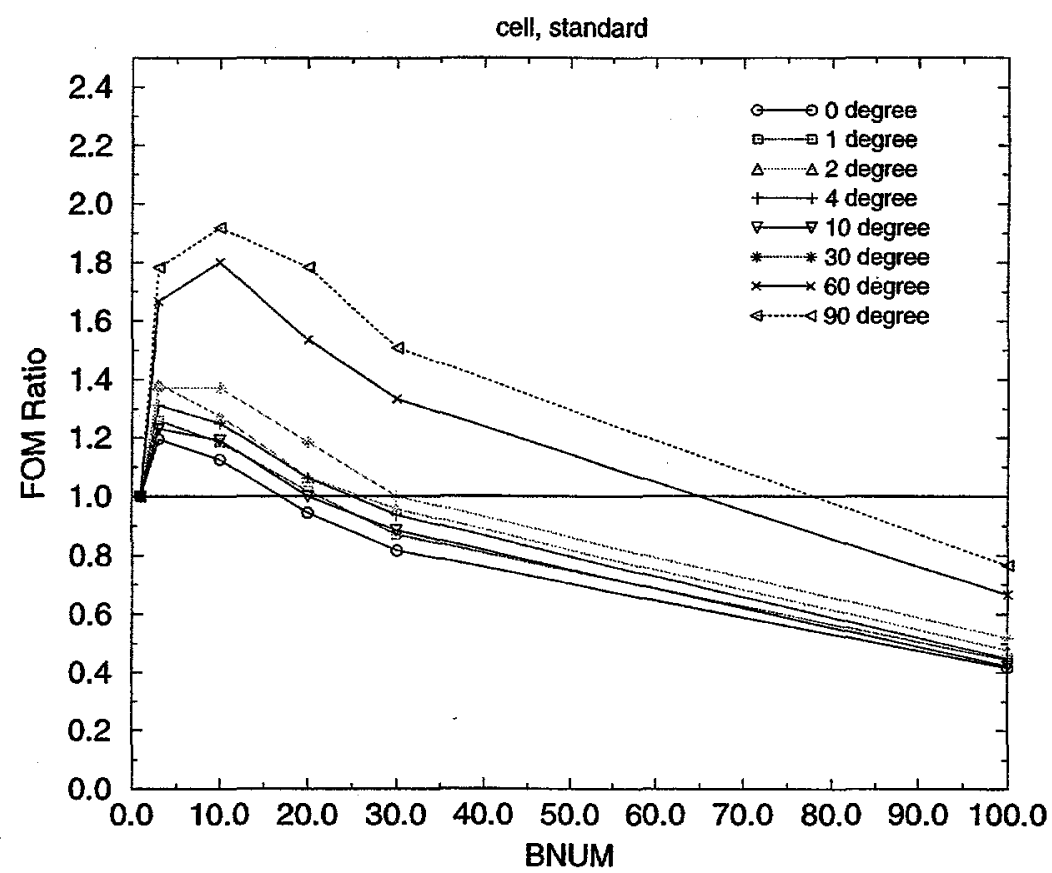

Fig. 38. Ratio of $\mathrm{FOM}_{\mathrm{i}}$ to $\mathrm{FOM}_{1}$ vs BNUM for standard splitting; cell tallies.

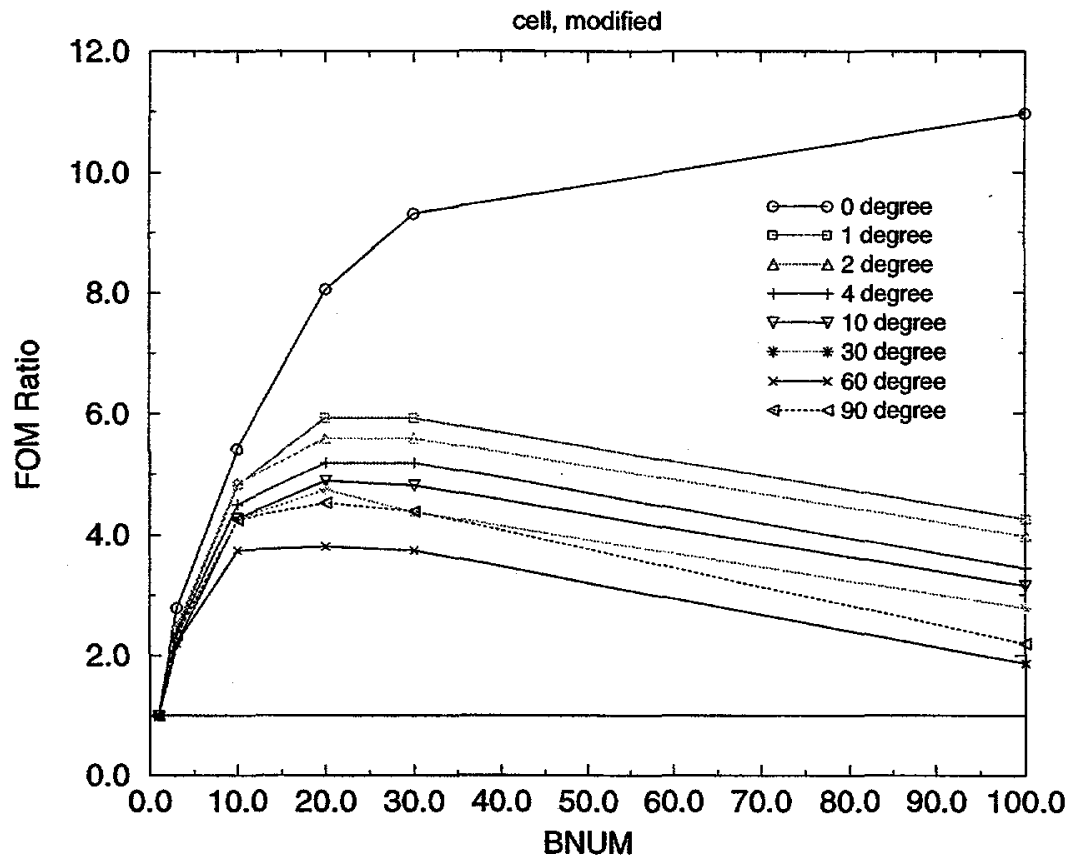

Fig. 39. Ratio of $\mathrm{FOM}_{\mathrm{i}}$ to $\mathrm{FOM}_{1}$ vs BNUM for modified splitting; cell tallies. 


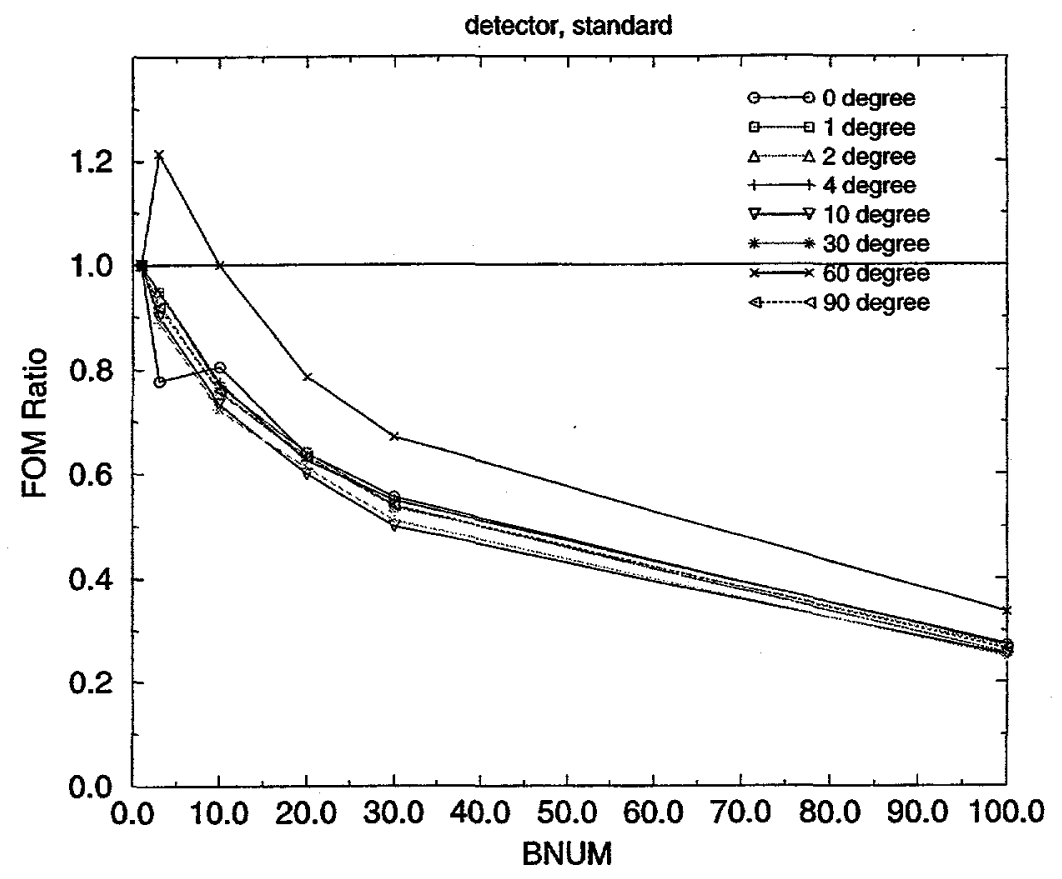

Fig. 40. Ratio of $\mathrm{FOM}_{\mathrm{i}}$ to $\mathrm{FOM}_{1}$ vs BNUM for standard splitting; detector tallies.

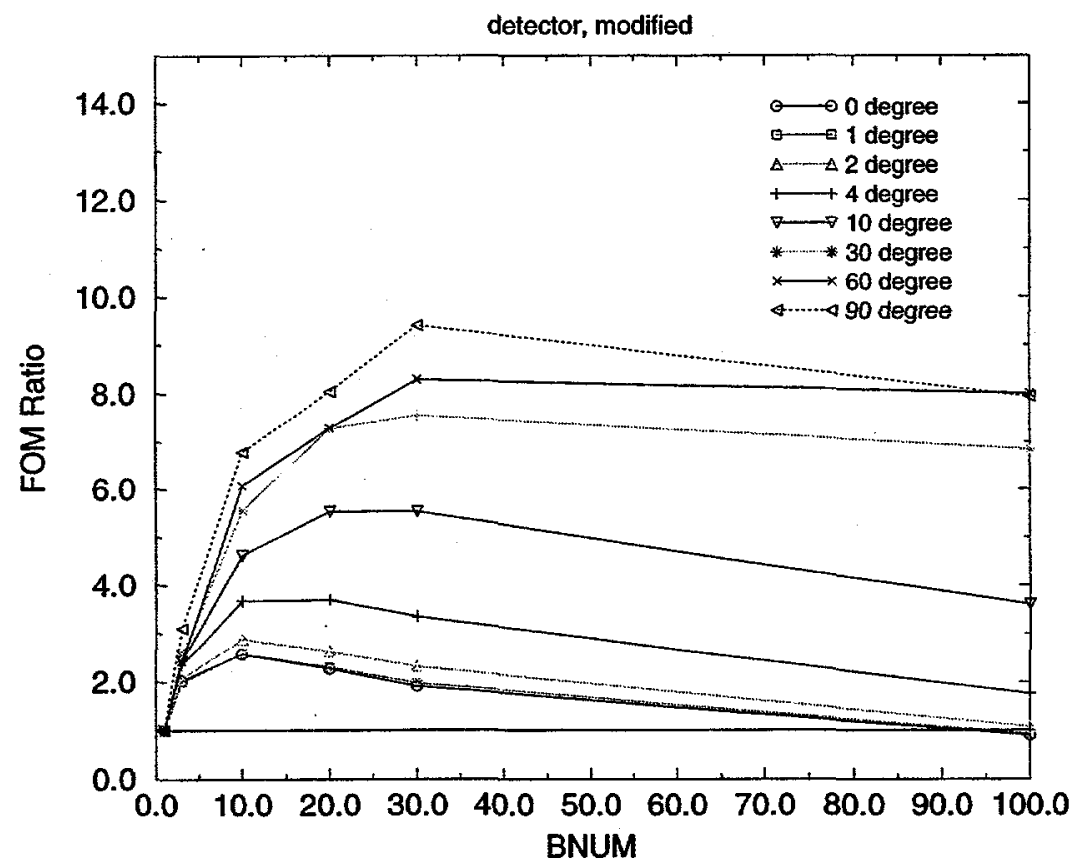

Fig. 41. Ratio of $\mathrm{FOM}_{\mathrm{i}}$ to $\mathrm{FOM}_{1}$ vs BNUM for standard splitting sampling; detector tallies. 


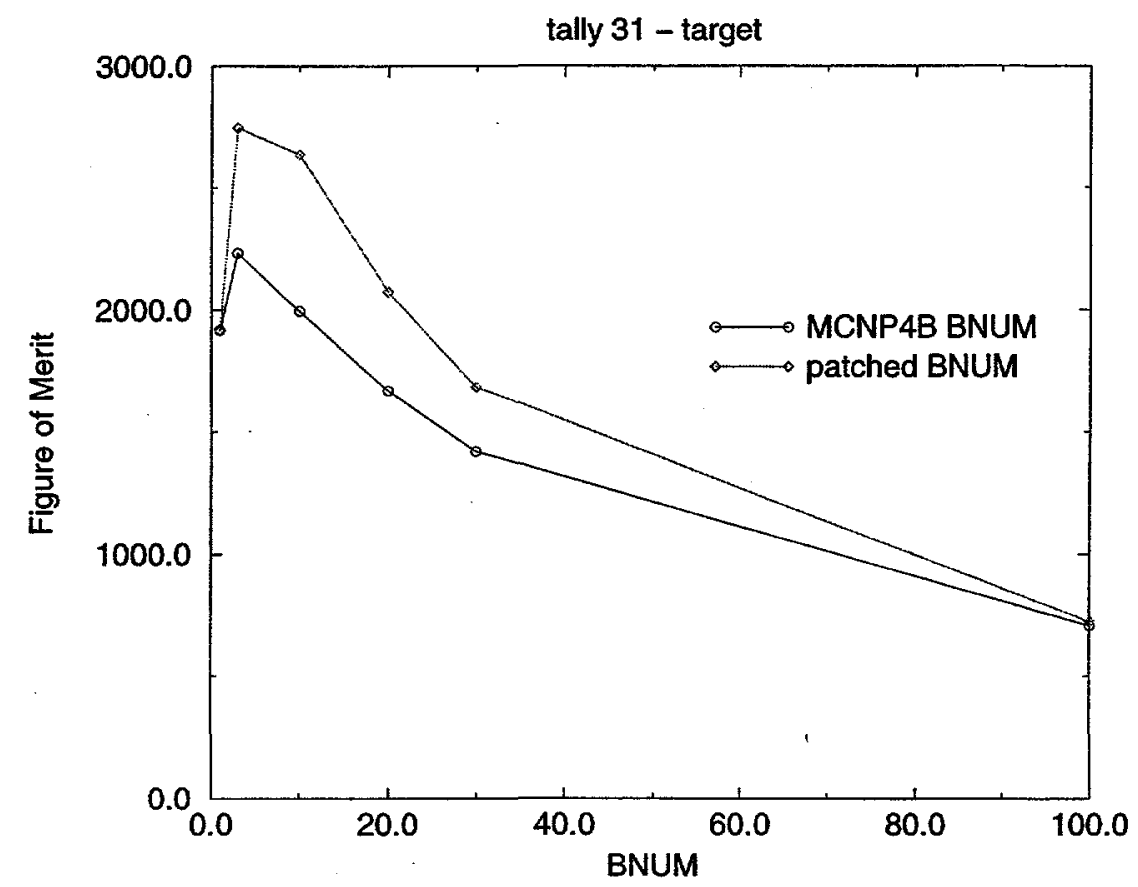

Fig. 42. FOM vs BNUM for photon current exiting the downstream face of the target in the forward direction.

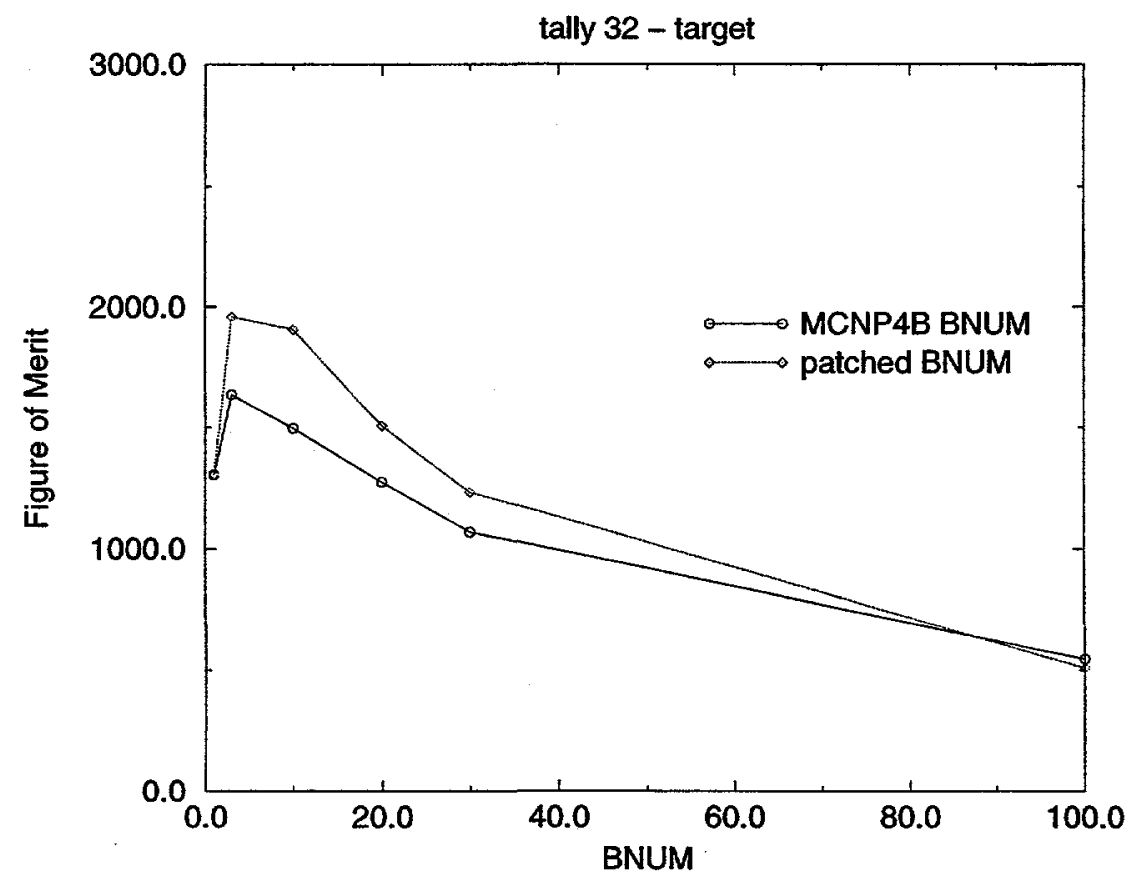

Fig. 43. FOM vs BNUM for photon flux tally of photons crossing the downstream face of the target. 
1. Simulation Geometry. The $10 \mathrm{~cm}$ thick disk was divided into cells $0.5 \mathrm{~cm}$ thick. The geometry is illustrated in Fig. 44. It should be noted that the disk is large in the $y$ and $z$ directions. The input file template is given in Appendix B.6. This problem is quite similar to the benchmarks described by Rogers and Bielajew. ${ }^{14}$

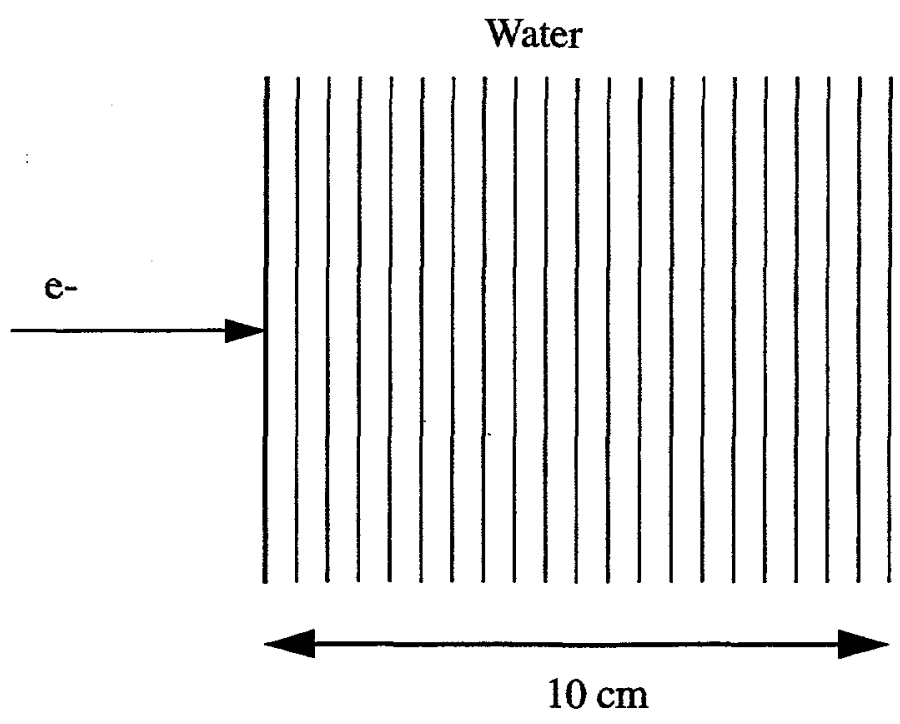

Fig. 44. Depth Dose Geometry.

2. Variation of Parameters. Several parameters were varied for this problem. These parameters, highlighted in the template input file (see Appendix B.6), were selected because they should impact the physics models. The problem was run in mode e, electrons only, or mode e p, electrons and photons. Also, several electron physics card parameters were varied. The IPHOT, ISTRG, and RNOK entries were either entered as 0 or 1 . Recall that for the integer parameters, 0 is the default, but RNOK has a default of 1 . A zero entry for IPHOT means that electrons will produce photons, while an entry of one means that electrons will not produce photons. For ISTRG=0, the straggling for electron energy loss is sampled, while for ISTRG=1, there is no straggling, and continuous slowing down energy loss is modeled. Knock-on electrons are produced when $\mathrm{RNOK}=1$, but not produced when $\mathrm{RNOK}=0$. The 18 th entry in the $\mathrm{DBCN}$ card was also varied. For an entry of one, the ITS3.0 energy grid sampling scheme is used, while for an entry of zero the default MCNP energy grid is used. Finally, the ESTEP parameter, which controls the number of electron substeps per energy step, was varied from the default of 3 to 15. 
Table XX serves a summary of the parameters used and the output for the each run. For the run designated inpdef, all of the input parameters are default values. This input file was run for MCNP version $4 \mathrm{a}$ as well as $4 \mathrm{xq}$. The first run listed mirrors the default run except that ESTEP is set to 15 . All other runs were performed with the default value of estep=3.

TABLE XX: INPUT PARAMETERS

\begin{tabular}{|c|c|c|c|c|c|}
\hline $\begin{array}{c}\text { Input } \\
\text { File }\end{array}$ & IPHOT & ISTRG & RNOK & $\begin{array}{c}\text { DBCN } \\
(\mathbf{1 8})\end{array}$ & MODE \\
\hline \hline inpe15 & 0 & 0 & 1 & 0 & $\mathrm{p} \mathrm{e}$ \\
\hline inpdef & 0 & 0 & 1 & 0 & $\mathrm{p} \mathrm{e}$ \\
\hline inp01 & 0 & 0 & 0 & 0 & $\mathrm{p} \mathrm{e}$ \\
\hline inp02 & 0 & 0 & 0 & 0 & $\mathrm{e}$ \\
\hline inp03 & 0 & 0 & 0 & 1 & $\mathrm{p} \mathrm{e}$ \\
\hline inp04 & 0 & 0 & 0 & 1 & $\mathrm{e}$ \\
\hline inpdef & 0 & 0 & 1 & 0 & $\mathrm{p} \mathrm{e}$ \\
\hline inp06 & 0 & 0 & 1 & 0 & $\mathrm{e}$ \\
\hline inp07 & 0 & 0 & 1 & 1 & $\mathrm{p} \mathrm{e}$ \\
\hline inp08 & 0 & 0 & 1 & 1 & $\mathrm{e}$ \\
\hline inp09 & 0 & 1 & 0 & 0 & $\mathrm{p} \mathrm{e}$ \\
\hline inp10 & 0 & 1 & 0 & 0 & $\mathrm{e}$ \\
\hline inp11 & 0 & 1 & 0 & 1 & $\mathrm{p} \mathrm{e}$ \\
\hline inp12 & 0 & 1 & 0 & 1 & $\mathrm{e}$ \\
\hline inp13 & 0 & 1 & 1 & 0 & $\mathrm{p} \mathrm{e}$ \\
\hline inp14 & 0 & 1 & 1 & 0 & $\mathrm{e}$ \\
\hline inp15 & 0 & 1 & 1 & 1 & $\mathrm{p} \mathrm{e}$ \\
\hline inp16 & 0 & 1 & 1 & 1 & $\mathrm{e}$ \\
\hline inp17 & 1 & 0 & 0 & 0 & $\mathrm{p} \mathrm{e}$ \\
\hline inp18 & 1 & 0 & 0 & 0 & $\mathrm{e}$ \\
\hline inp19 & 1 & 0 & 0 & 1 & $\mathrm{pe}$ \\
\hline inp20 & 1 & 0 & 0 & 1 & $\mathrm{e}$ \\
\hline inp21 & 1 & 0 & 1 & 0 & $\mathrm{pe}$ \\
\hline inp22 & 1 & 0 & 1 & 0 & $\mathrm{e}$ \\
\hline inp23 & 1 & 0 & 1 & 1 & $\mathrm{p} \mathrm{e}$ \\
\hline inp24 & 1 & 0 & 1 & 1 & $\mathrm{e}$ \\
\hline
\end{tabular}


TABLE XX: INPUT PARAMETERS (CONT.)

\begin{tabular}{|c|c|c|c|c|c|}
\hline $\begin{array}{c}\text { Input } \\
\text { File }\end{array}$ & IPHOT & ISTRG & RNOK & $\begin{array}{c}\text { DBCN } \\
(\mathbf{1 8})\end{array}$ & MODE \\
\hline \hline inp25 & 1 & 1 & 0 & 0 & $\mathrm{p} \mathrm{e}$ \\
\hline inp26 & 1 & 1 & 0 & 0 & $\mathrm{e}$ \\
\hline inp27 & 1 & 1 & 0 & 1 & $\mathrm{p} \mathrm{e}$ \\
\hline inp28 & 1 & 1 & 0 & 1 & $\mathrm{e}$ \\
\hline inp29 & 1 & 1 & 1 & 0 & $\mathrm{p} \mathrm{e}$ \\
\hline inp30 & 1 & 1 & 1 & 0 & $\mathrm{e}$ \\
\hline inp31 & 1 & 1 & 1 & 1 & $\mathrm{p} \mathrm{e}$ \\
\hline inp32 & 1 & 1 & 1 & 1 & $\mathrm{e}$ \\
\hline
\end{tabular}

3. Problem Tallies. The main item of interest in this problem was energy deposition, which was tallied with a *F8 tally. A pulse height tally (F8) was used to record the electron and photon energy deposition spectra in each $0.5 \mathrm{~cm}$ water cell. The charge deposition in each cell was tallied as well. The electron and photon currents, in terms of weight and energy, were also tallied for each of the water cell surfaces. The electron and photon fluxes were also tallied for each water cell. For electrons, the flux averaged over each of the cells, in terms of weight and energy, was also tallied.

4. Convergence of Results. Tally fluctuation bins were used to track the convergence of the tallies. For the energy and charge deposition tallies, the last bin $(9.5-10 \mathrm{~cm}$ depth) was used to check for convergence. For the current (F1) and flux (F2) tallies, the first cosine bin ( -1 to 0$)$ and the first surface $(x=0.0)$ were used as tally checks. For the flux averaged over a cell $(F 4)$, the last cell was checked $(9.5-10 \mathrm{~cm}$ depth). These particular bins were chosen because they were deemed to be the least likely to converge. If they converged, then the other bins should definitely converge. In most cases, the tallies passed all ten of the statistical checks. The most common tallies not to pass all of the checks were the electron flux averaged over the last cell, and the charge deposition in the last cell. Of course, there were relatively fewer tallies in these cells, since most of the electrons failed to penetrate that deeply into the water. Furthermore, since the tally fluctuation bins were chosen in such a conservative manner, not passing the checks does not necessarily indicate problems with the overall tally. 
5. Results. The graphs presented next show the energy deposition as a function of distance in the water disk. The default values are: electrons will produce photons, sampled straggling for energy loss, knock-on electrons are produced, the MCNP energy grid is used, and both electrons and photons are included. The graphs show two curves: the default run, and a run with some parameter(s) different from the default. The title indicates the parameters that differed from the default, as well as the mode of the non-default run.

6. Timing Studies. It was interesting to note how the cutoff energy affected the speed of the calculation. All of the problems were run with an electron cutoff energy of $189 \mathrm{keV}$. For comparison purposes, inp02 was run with two other cutoff values. The results are presented in the following table. It should be noted that the speed of the calculation is not linearly related to the cutoff energy of the problem. The figure of merit for the electron energy deposition tally (*F8:e) behaves in a manner quite similar to the particles run per minute.

7. Conclusions. A few conclusions can be drawn from the results. For cases with no straggling, there is a slight dose buildup near the incident surface, and much less energy deposited near the end of the slab. These results agree with the no-straggling results of Rogers and Bielajew (cf Figure 6.7, Ref. 14), who used EGS4. When knock-on electrons are not included, there is more energy deposited early in the water slab, since more energy can be deposited locally and not be carried away by the knock-ons. This differs slightly from the results of Rogers and Bielajew (cf Figure 6.7, Ref. 14), but is in agreement with the results of Nahum (cf Figure 1.11, Ref. 15). Changing the mode from electrons and photons to electrons only, for any case, tends to increase the energy deposited near the beginning of the slab, and decrease the energy deposited at the end of the slab. This can be explained because in electron mode only, any bremsstrahlung energy is deposited locally, since no photons are created. When the nearest bin energy sampling (used in ITS) energy grid is used, there is more energy deposited early in the water slab, whereas there is less energy later in the slab. This implies that there is more scatter in the ITS methodology. For cases where the electrons do not produce photons, the electron-only mode and the electron and photon mode agree. The CSDA results show that energy is deposited past the electron range of $9.23 \mathrm{~cm}$ calculated with the total stopping power because even though collisional straggling is turned off, there is still radiative straggling which can effectively allow the electron to lose less energy due to radiative processes. 
TABLE XXI: TIMING COMPARISON

\begin{tabular}{|c|c|c|}
\hline $\begin{array}{c}\text { Cutoff Energy } \\
\text { (keV) }\end{array}$ & $\begin{array}{c}\text { Particles per } \\
\text { minute }\end{array}$ & $\begin{array}{c}\text { Figure of Merit, } \\
\text { *F8:e }\end{array}$ \\
\hline \hline 189 & 5495 & 588 \\
\hline 10 & 3684 & 397 \\
\hline 1 & 2957 & 319 \\
\hline
\end{tabular}

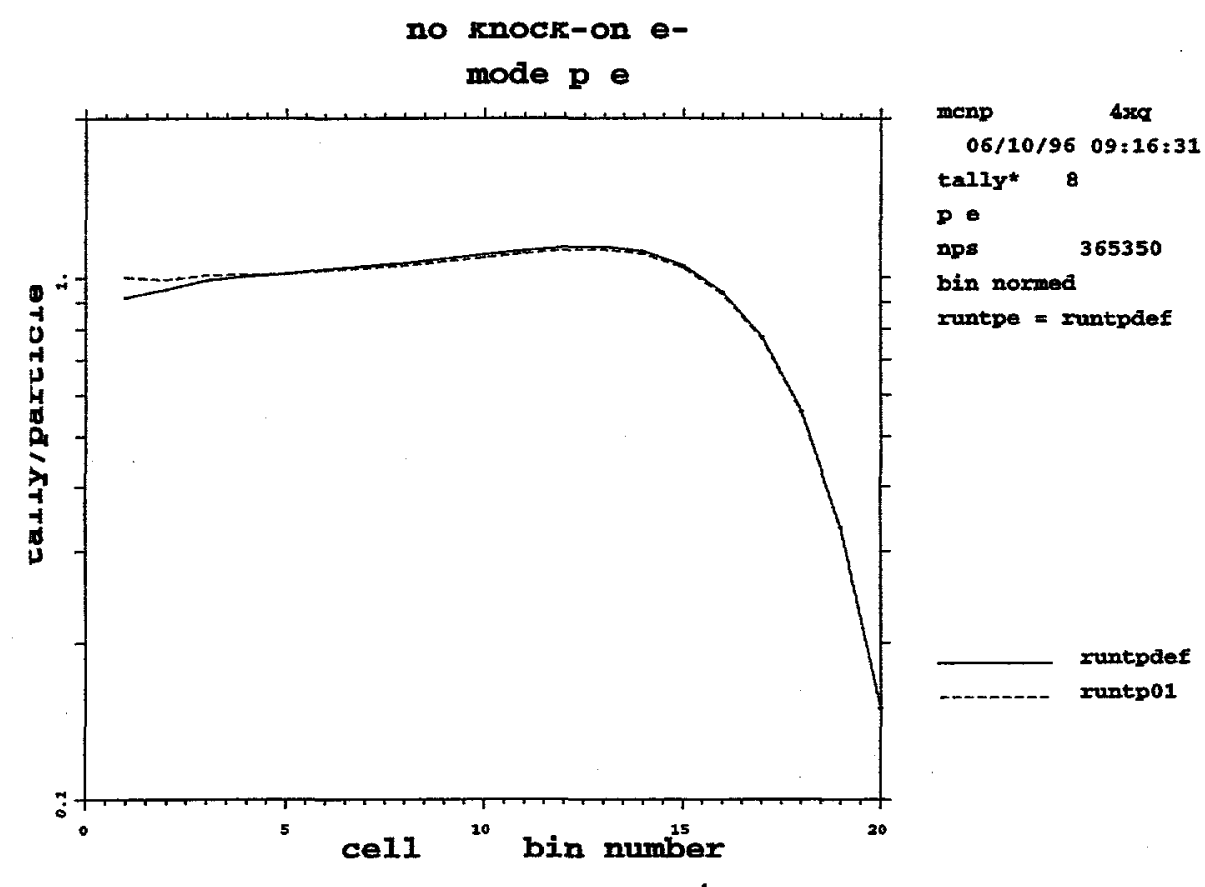

Fig. 45. The solid line indicates default settings. The dashed line indicates the same settings, except that there are no knock-on electrons. In both cases, the problem includes photons and electrons. 


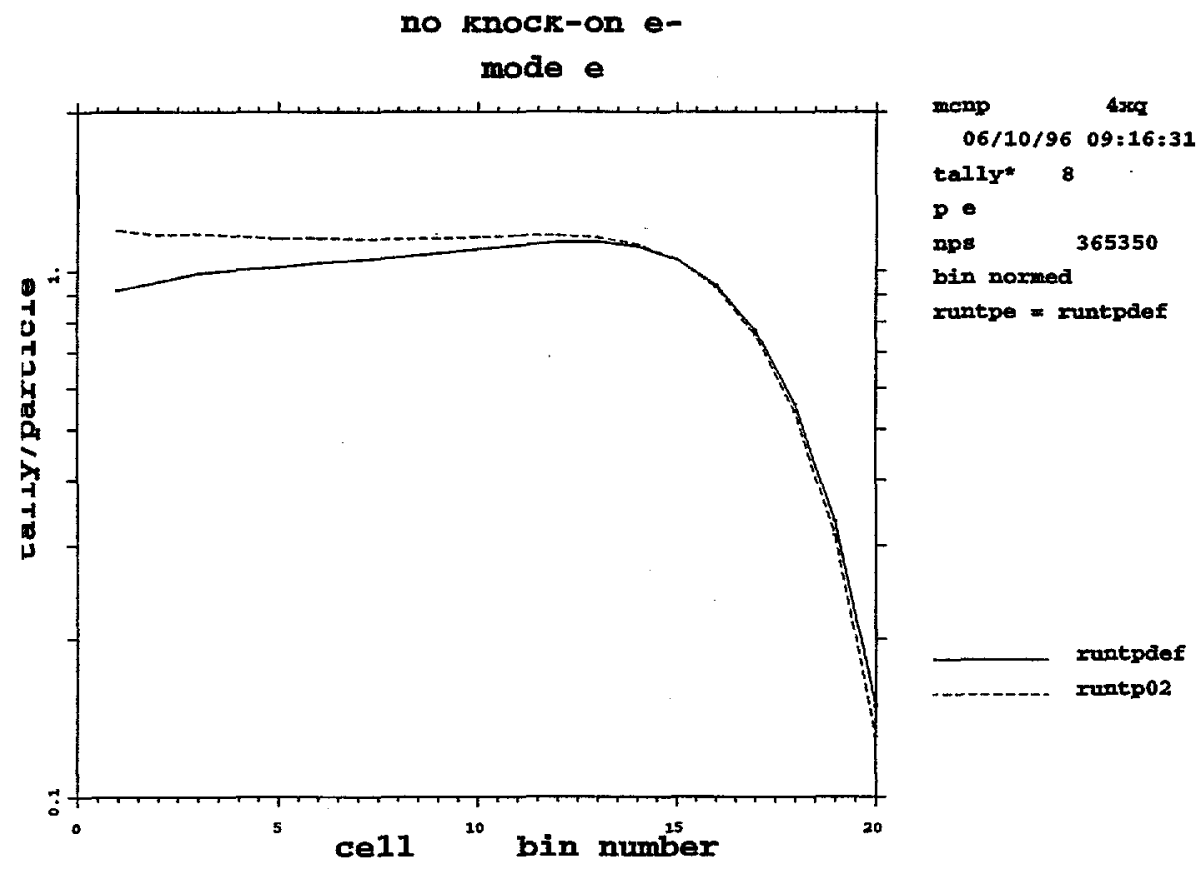

Fig. 46. The solid line indicates default settings. The dashed line indicates the same settings, except that there are no knock-on electrons. For the default case, the problem includes photons and electrons, whereas only electrons are included in the other case.

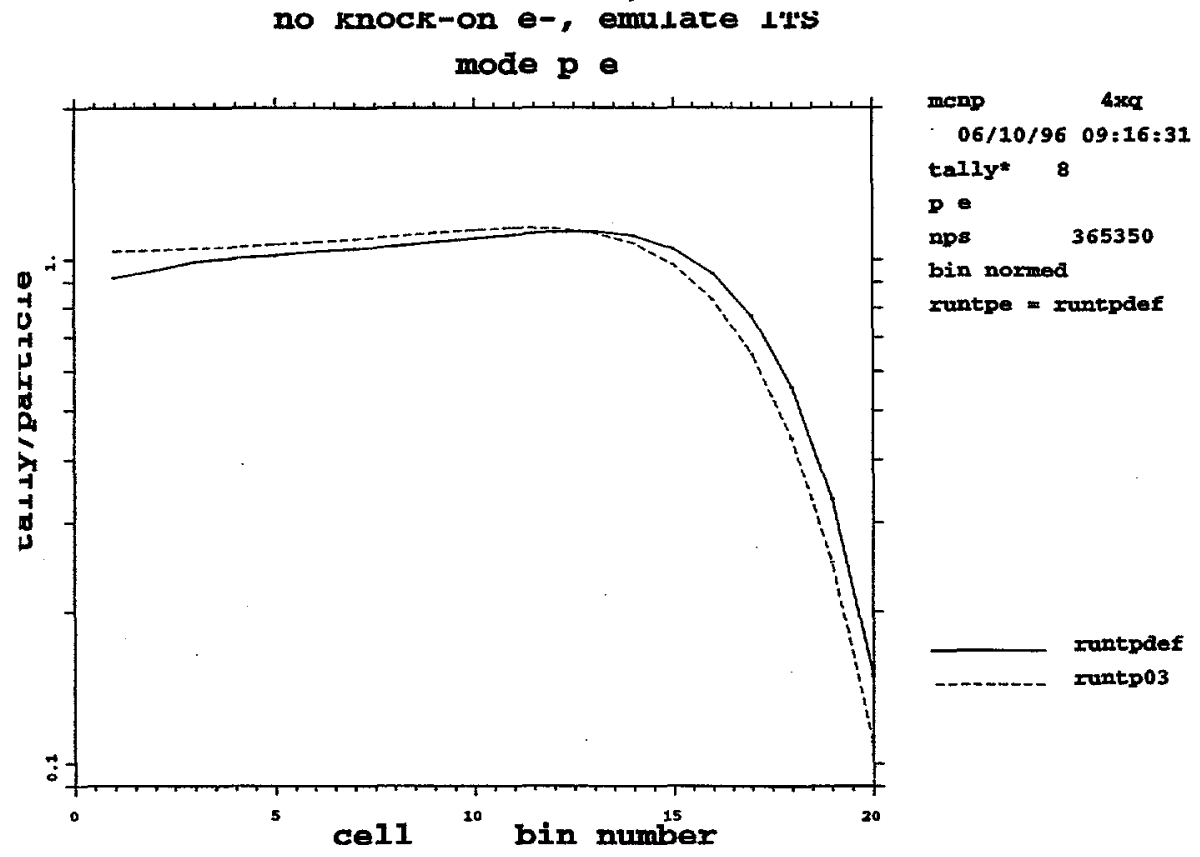

Fig. 47. The solid line indicates default settings. The dashed line indicates the same settings, except that there are no knock-on electrons, and the ITS energy grid is used. In both cases, the problem includes photons and electrons. 


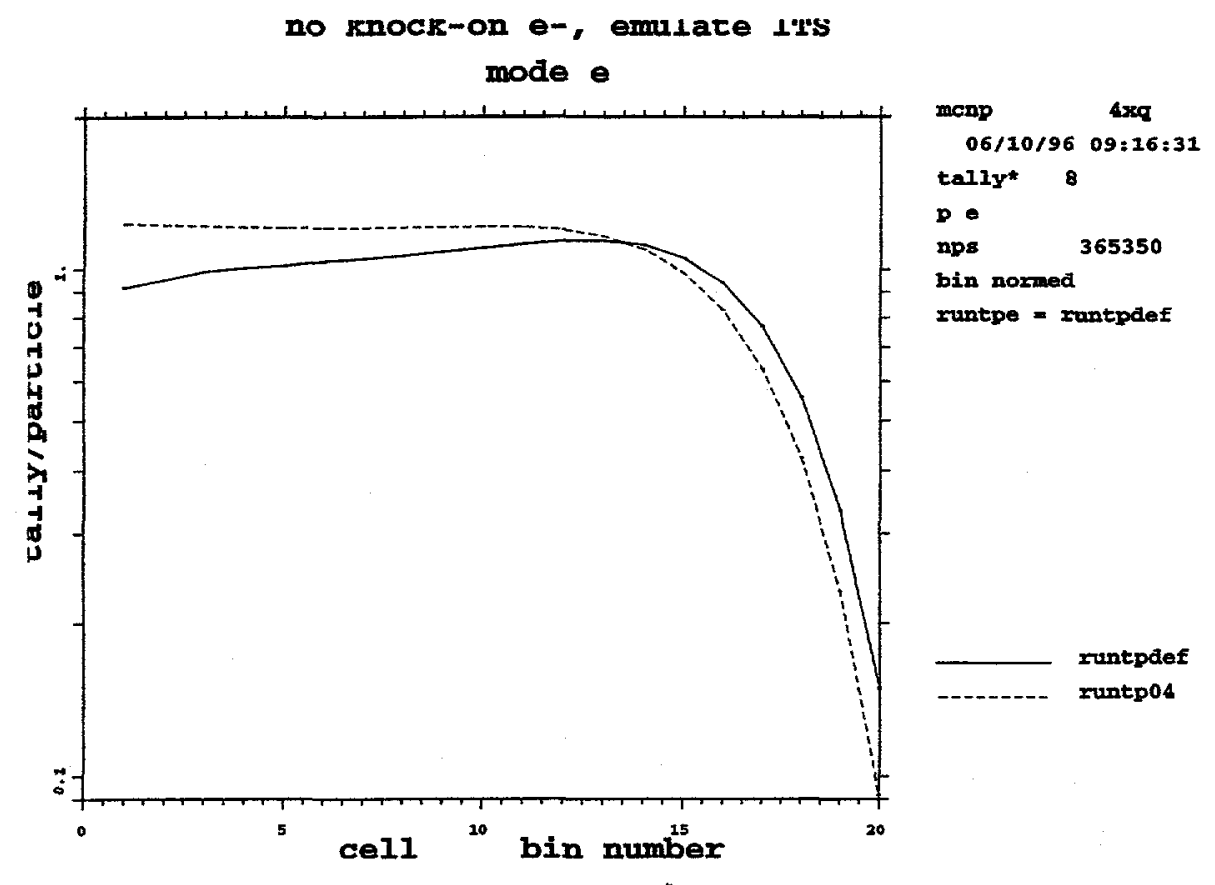

Fig. 48. The solid line indicates default settings. The dashed line indicates the same settings, except that there are no knock-on electrons, and the ITS energy grid is used. For the default case, the problem includes photons and electrons, whereas only electrons are included in the other case.

ait deraulcs, excpec mode e

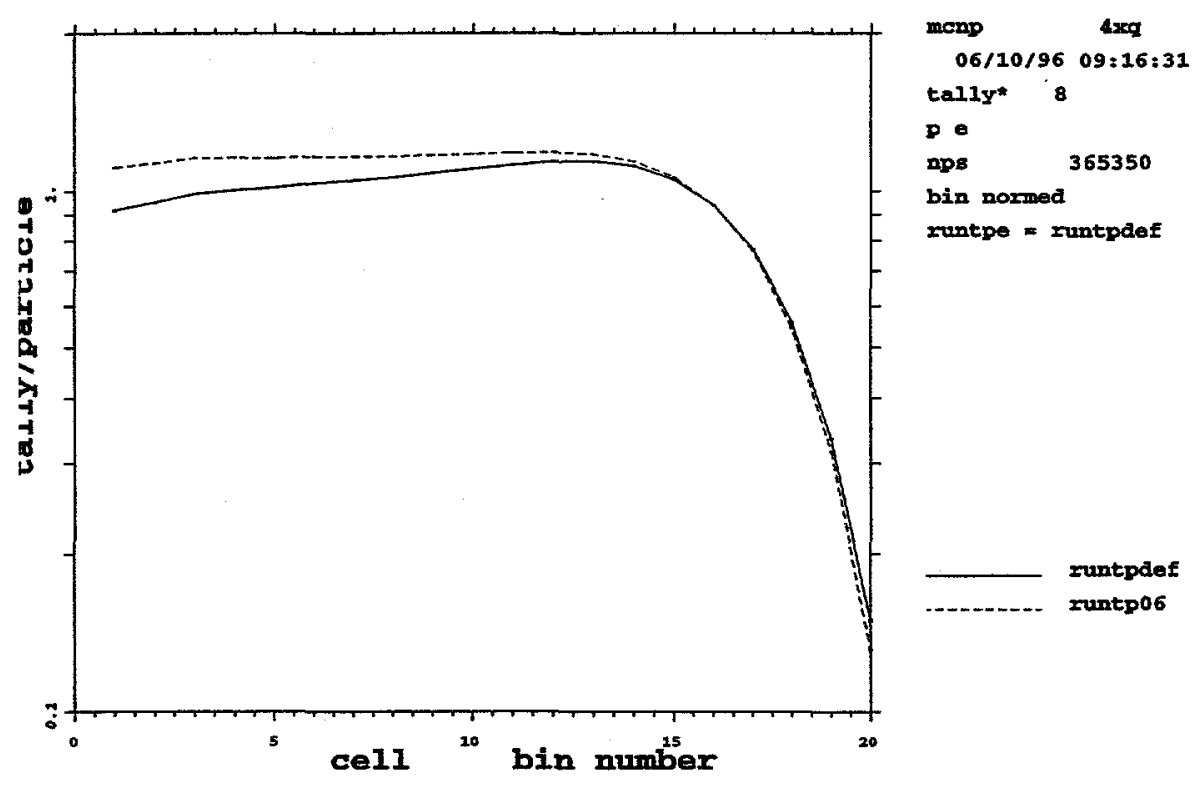

Fig. 49. The solid line indicates all default settings. The dashed line indicates all default settings, except the problem only includes electrons. 


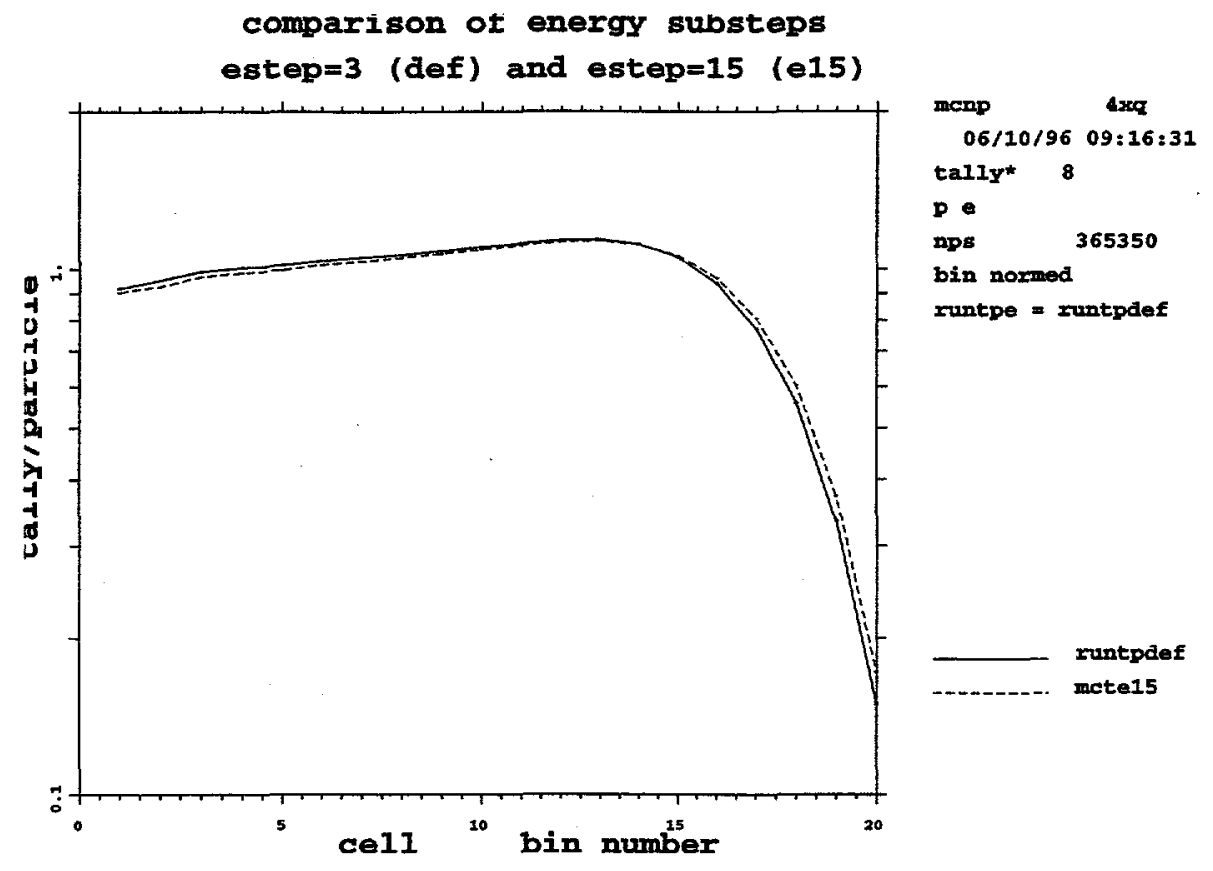

Fig. 50. Both runs have default settings, except for ESTEP. The solid line is estep=3 (default), whereas the dashed line is ESTEP $=15$.

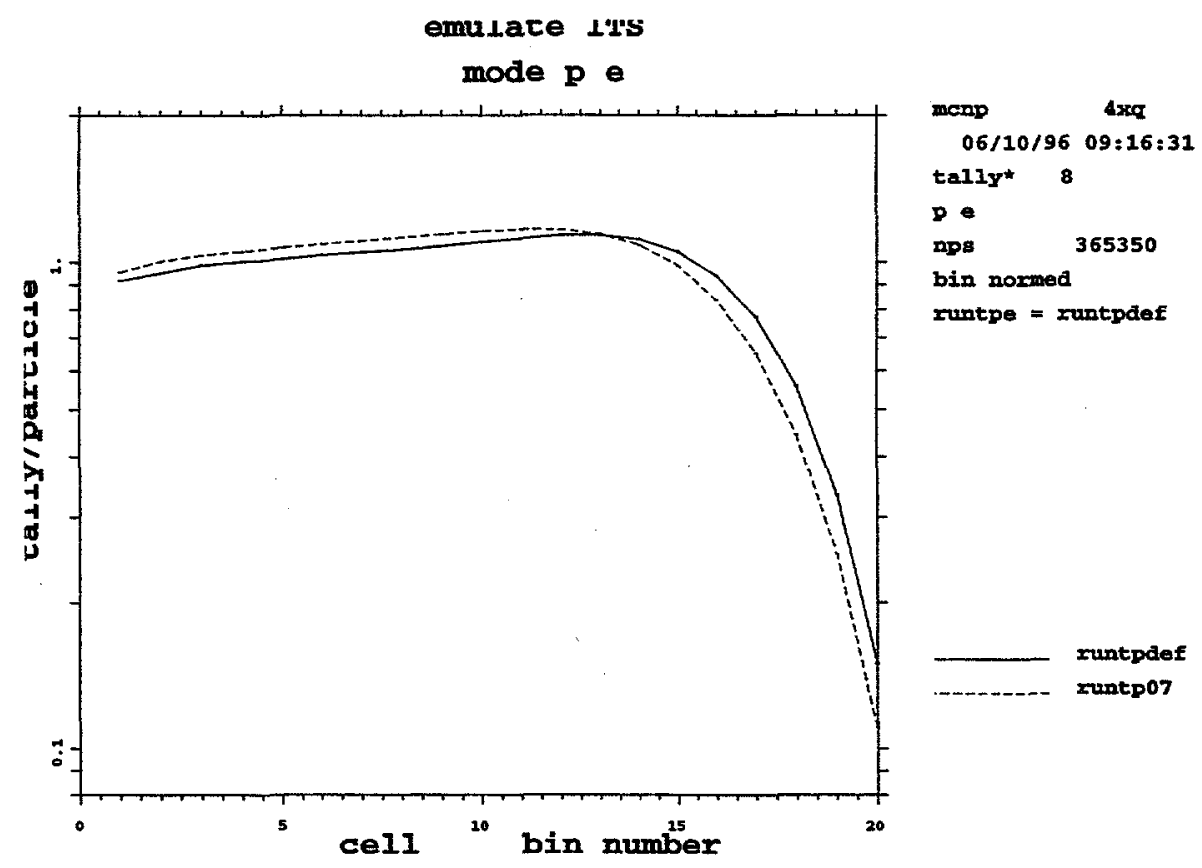

Fig. 51. The solid line indicates default settings. The dashed line indicates the same settings, except that the ITS energy grid is used. In both cases, the problem includes photons and electrons. 


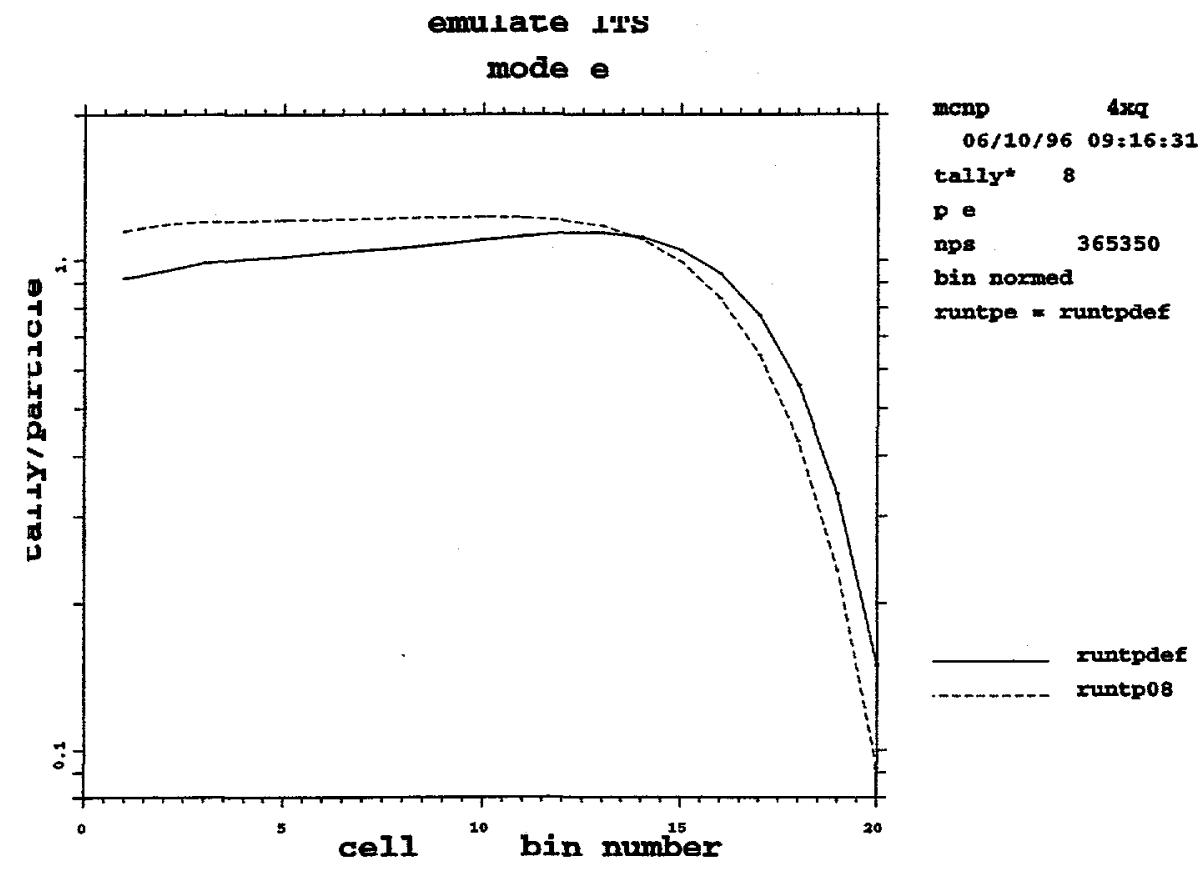

Fig. 52. The solid line indicates default settings. The dashed line indicates the same settings, except that the ITS energy grid is used. For the default case, the problem includes photons and electrons, whereas only electrons are included in the other case.

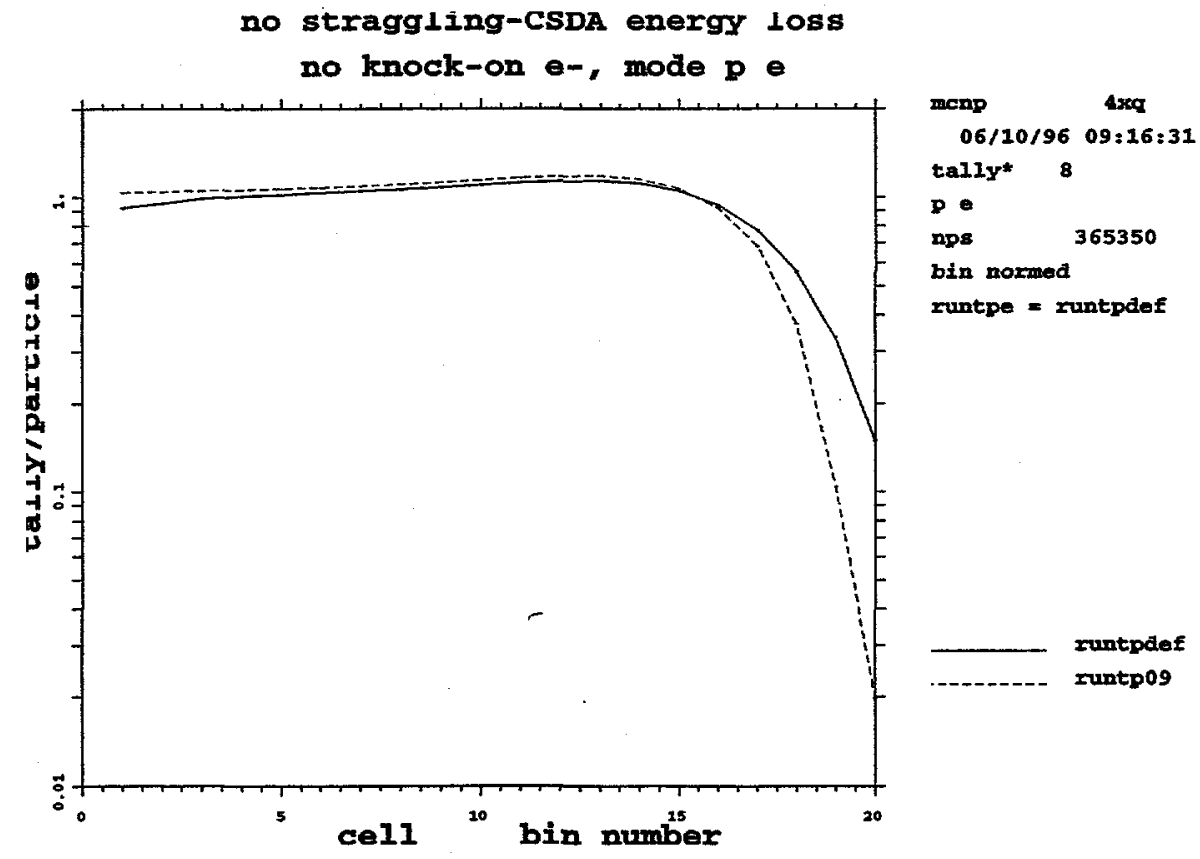

Fig. 53. The solid line indicates default settings. The dashed line indicates the same settings, except that there is no straggling, i.e., CSDA energy loss, and no knock-on electrons. In both cases, the problem includes photons and electrons. 


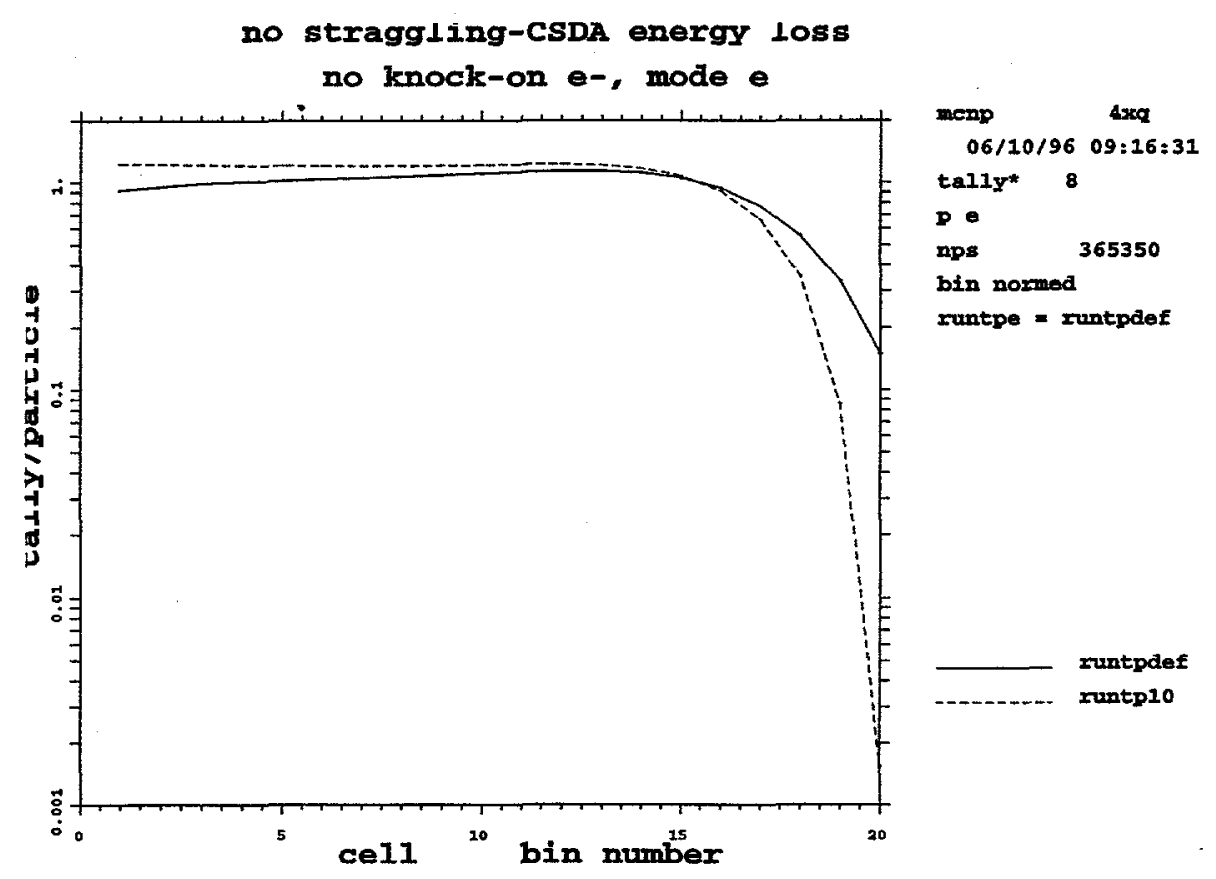

Fig. 54. The solid line indicates default settings. The dashed line indicates the same settings, except that there is CSDA energy loss, and no knock-on electrons. For the default case, the problem includes photons and electrons, whereas only electrons are included in the other case.

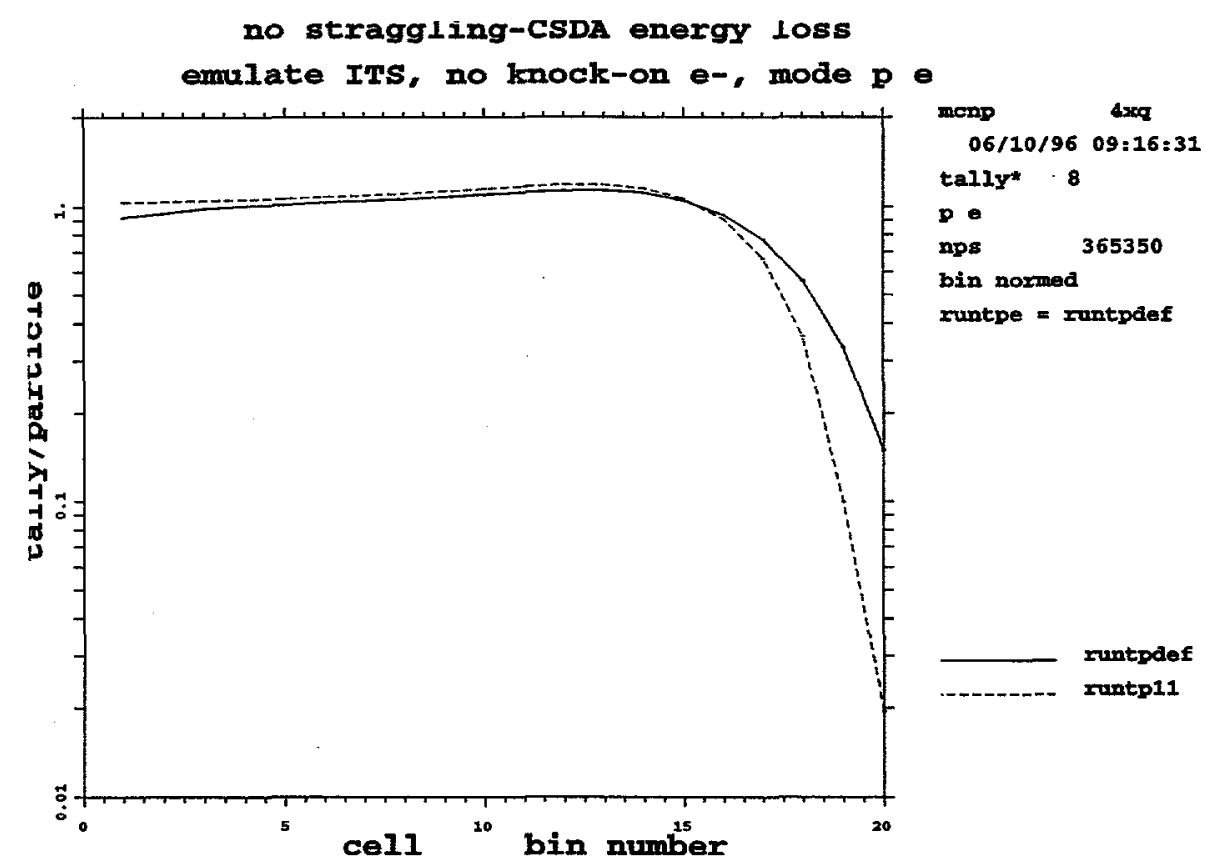

Fig. 55. The solid line indicates default settings. The dashed line indicates the same settings, except that there is CSDA energy loss, the ITS energy grid is used, and there are no knock-on electrons. In both cases, the problem includes photons and electrons. 


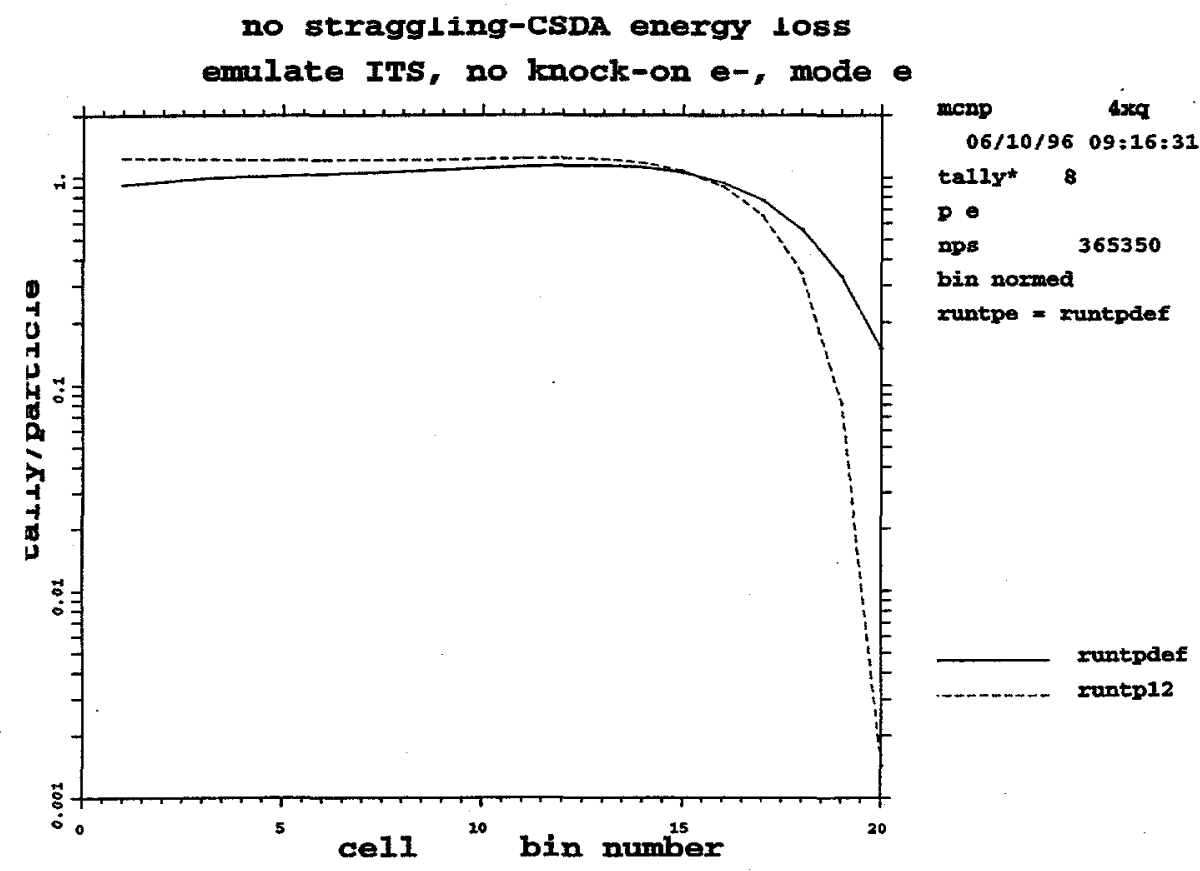

Fig. 56. The solid line indicates default settings. The dashed line indicates the same settings, except that there is CSDA energy loss, the ITS energy grid is used, and no knock-on electrons. For the default case, the problem includes photons and electrons, whereas only electrons are included in the other case.

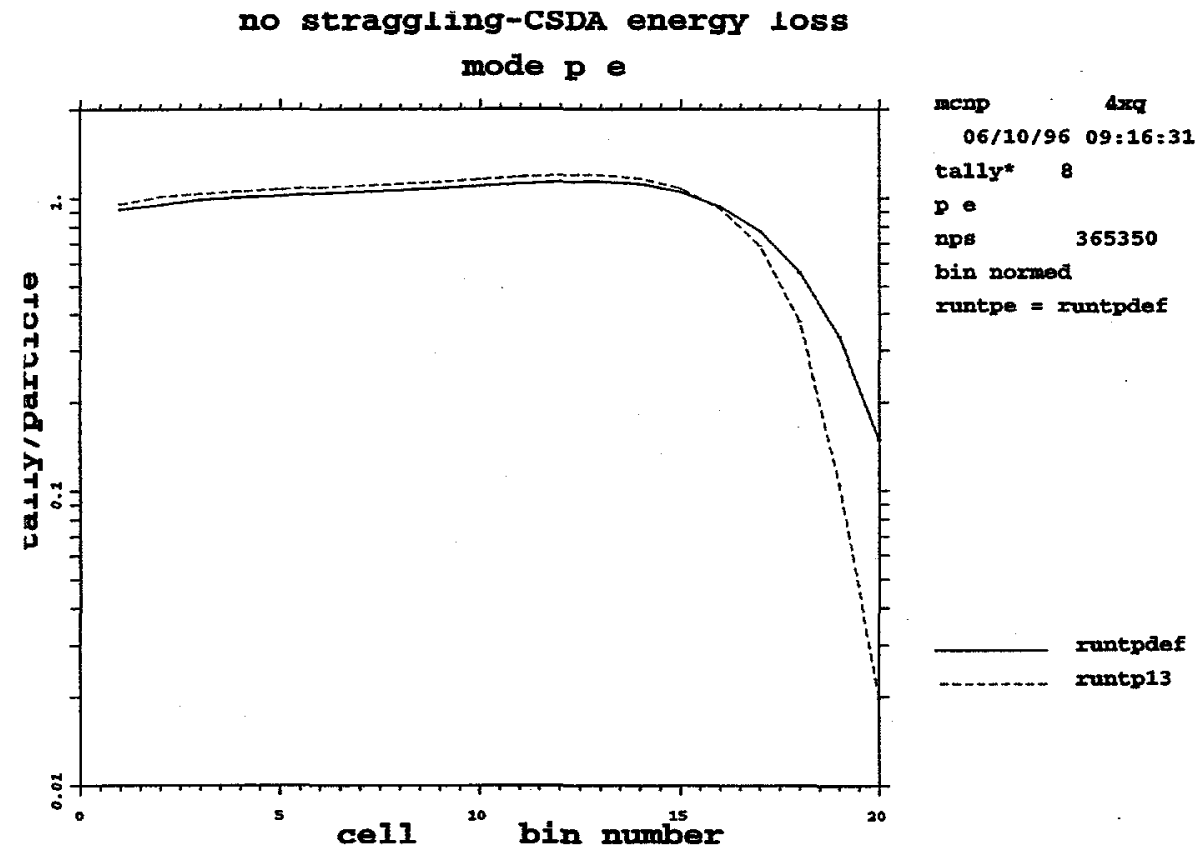

Fig. 57. The solid line indicates default settings. The dashed line indicates the same settings, except that there is CSDA energy loss. In both cases, the problem includes photons and electrons. 


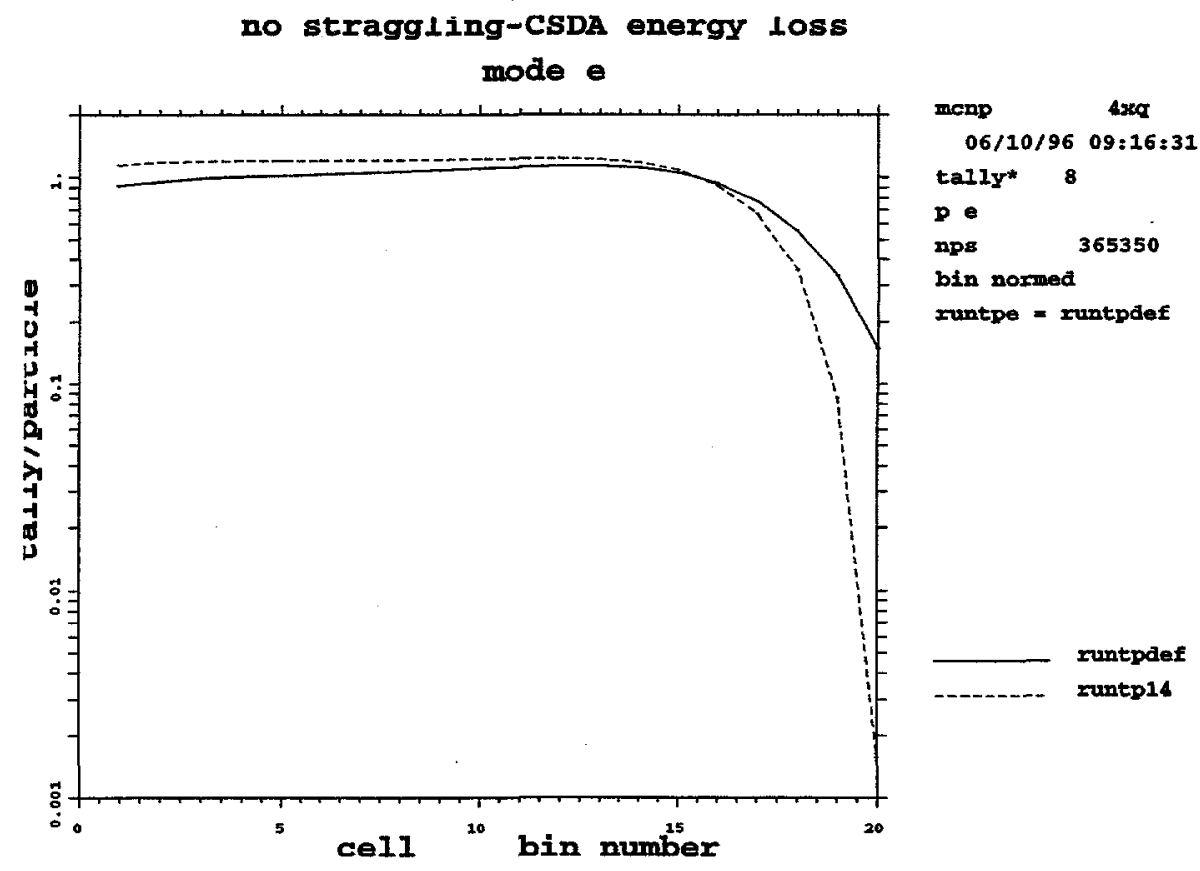

Fig. 58. The solid line indicates default settings. The dashed line indicates the same settings, except that there is CSDA energy loss. For the default case, the problem includes photons and electrons, whereas only electrons are included in the other case.

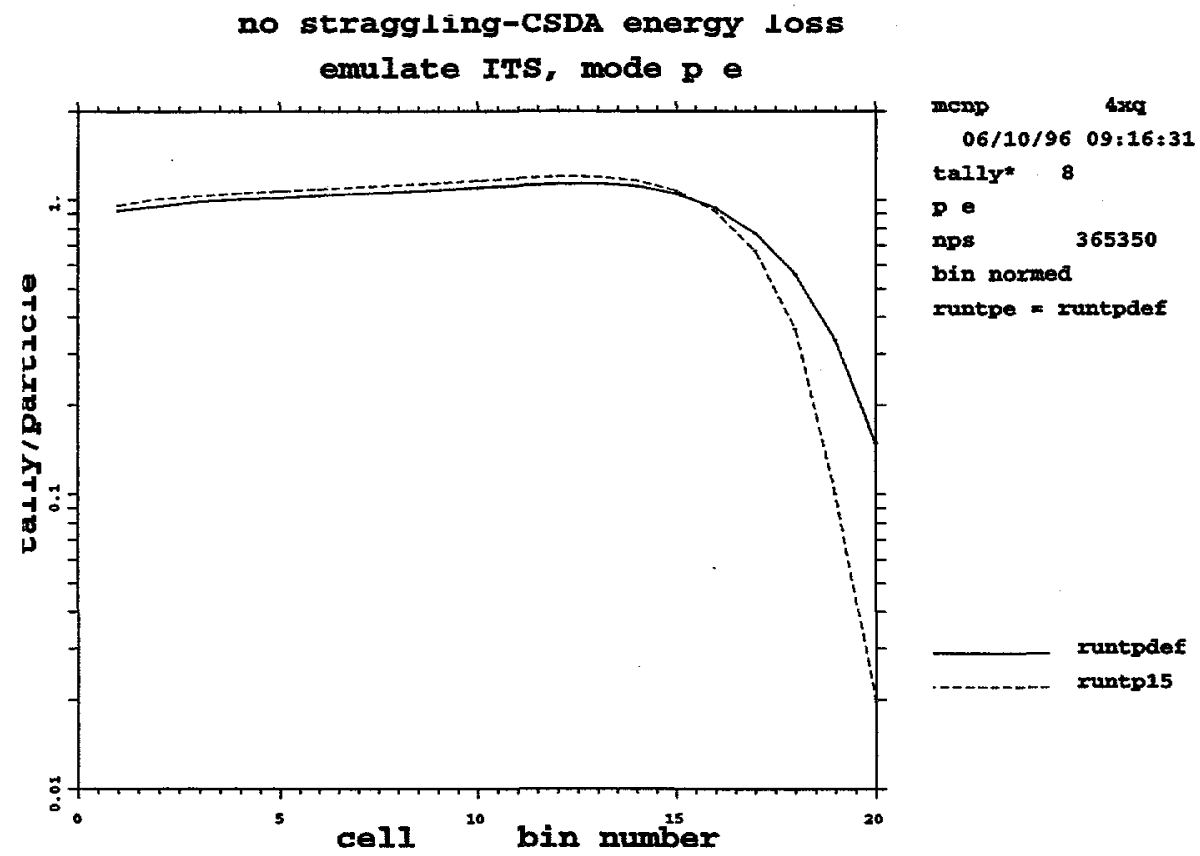

Fig. 59. The solid line indicates default settings. The dashed line indicates the same settings, except that there is CSDA energy loss, and the ITS energy grid is used. In both cases, the problem includes photons and electrons. 


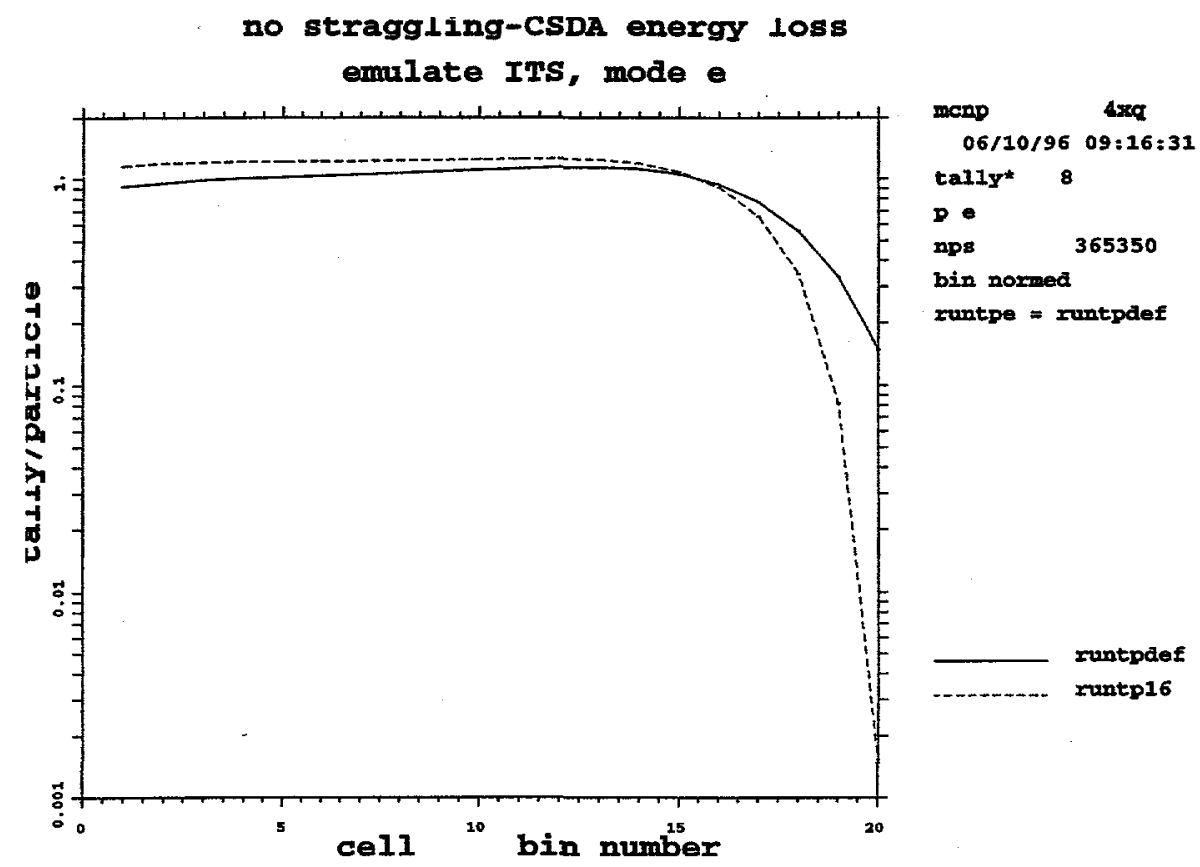

Fig. 60. The solid line indicates default settings. The dashed line indicates the same settings, except that there is CSDA energy loss, and the ITS energy grid is used. For the default case, the problem includes photons and electrons, whereas only electrons are included in the other case.

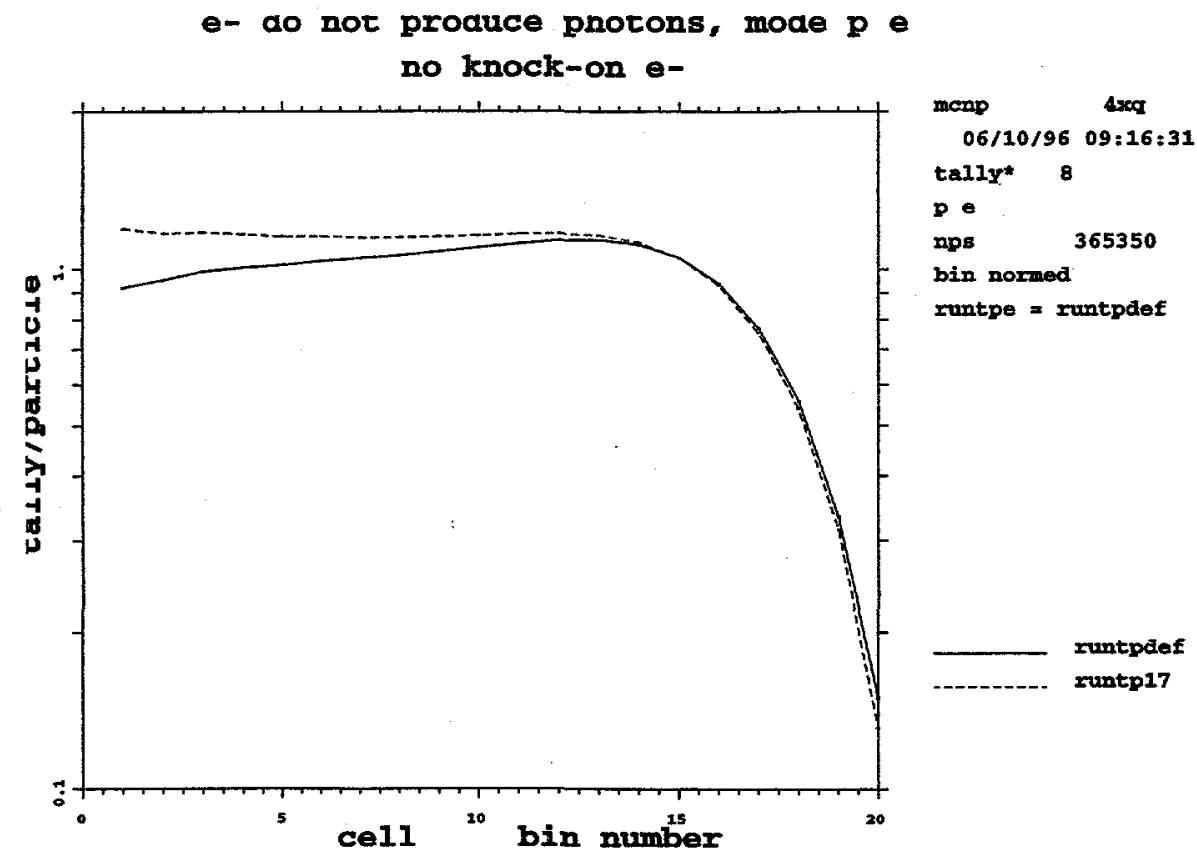

Fig. 61. The solid line indicates default settings. The dashed line indicates the same settings, except that electrons do not produce photons, and there are no knock-on electrons. In both cases, the problem includes photons and electrons. 


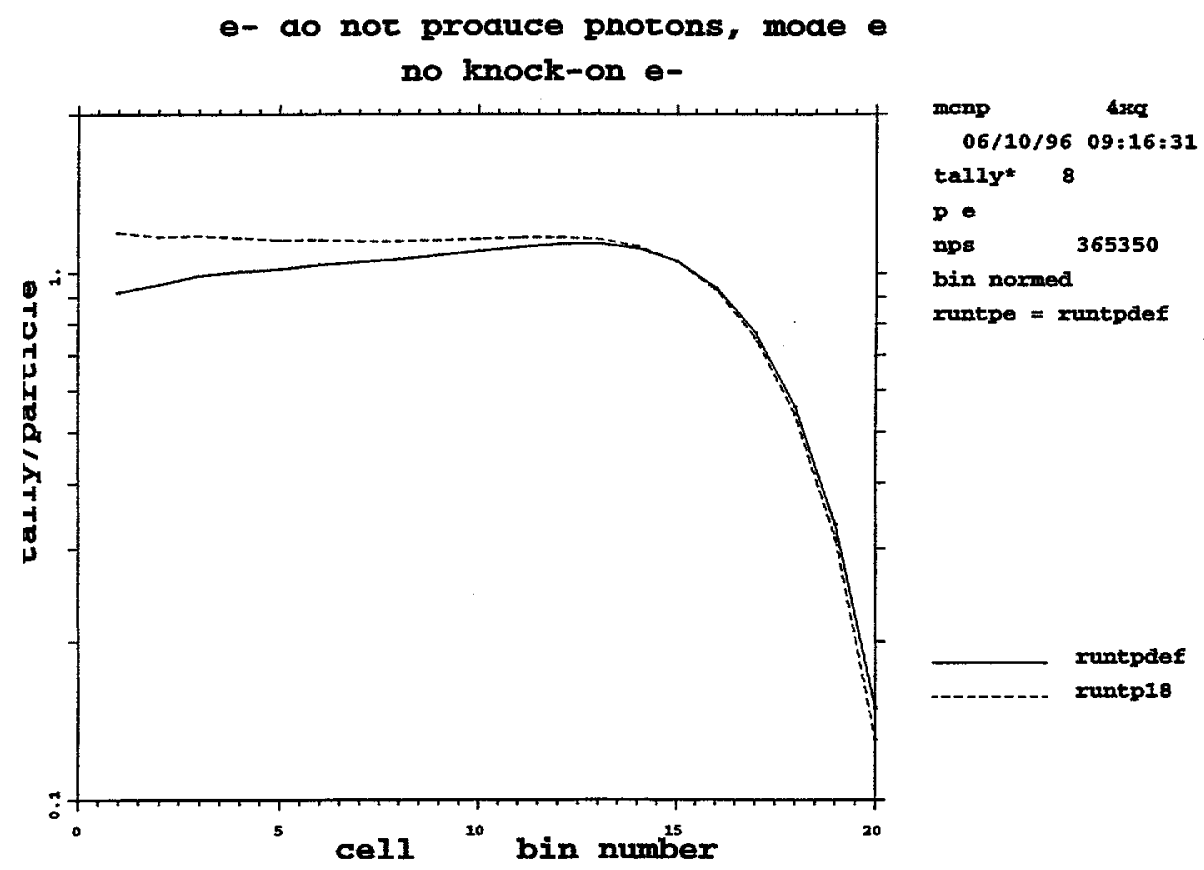

Fig. 62. The solid line indicates default settings. The dashed line indicates the same settings, except that electrons do not produce photons, and there are no knock-on electrons. For the default case, the problem includes photons and electrons, whereas only electrons are included in the other case.

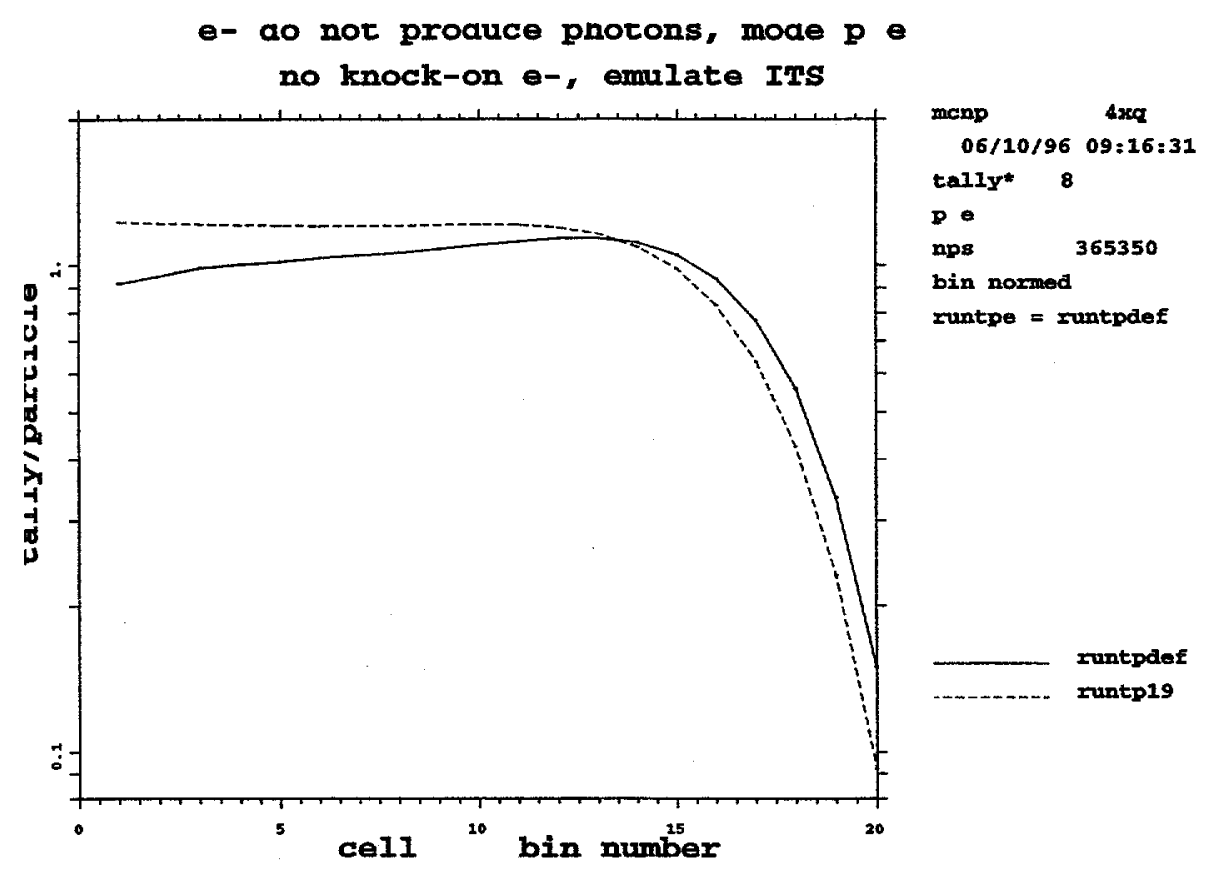

Fig. 63. The solid line indicates default settings. The dashed line indicates the same settings, except that electrons do not produce photons, there are no knock-on electrons, and the ITS energy grid is used. In both cases, the problem includes photons and electrons. 


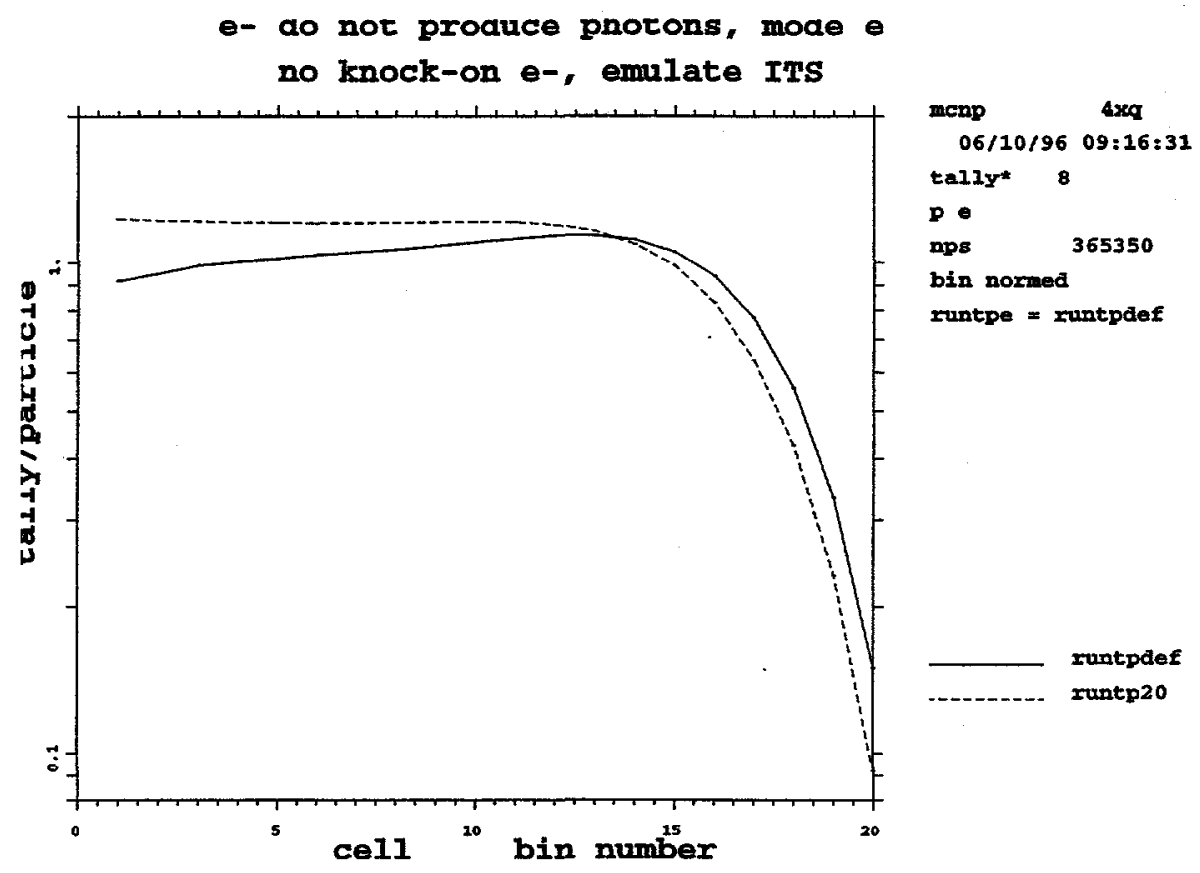

Fig. 64. The solid line indicates default settings. The dashed line indicates the same settings, except that electrons do not produce photons, there are no knock-on electrons, and the ITS energy grid is used. For the default case, the problem includes photons and electrons, whereas only electrons are included in the other case.

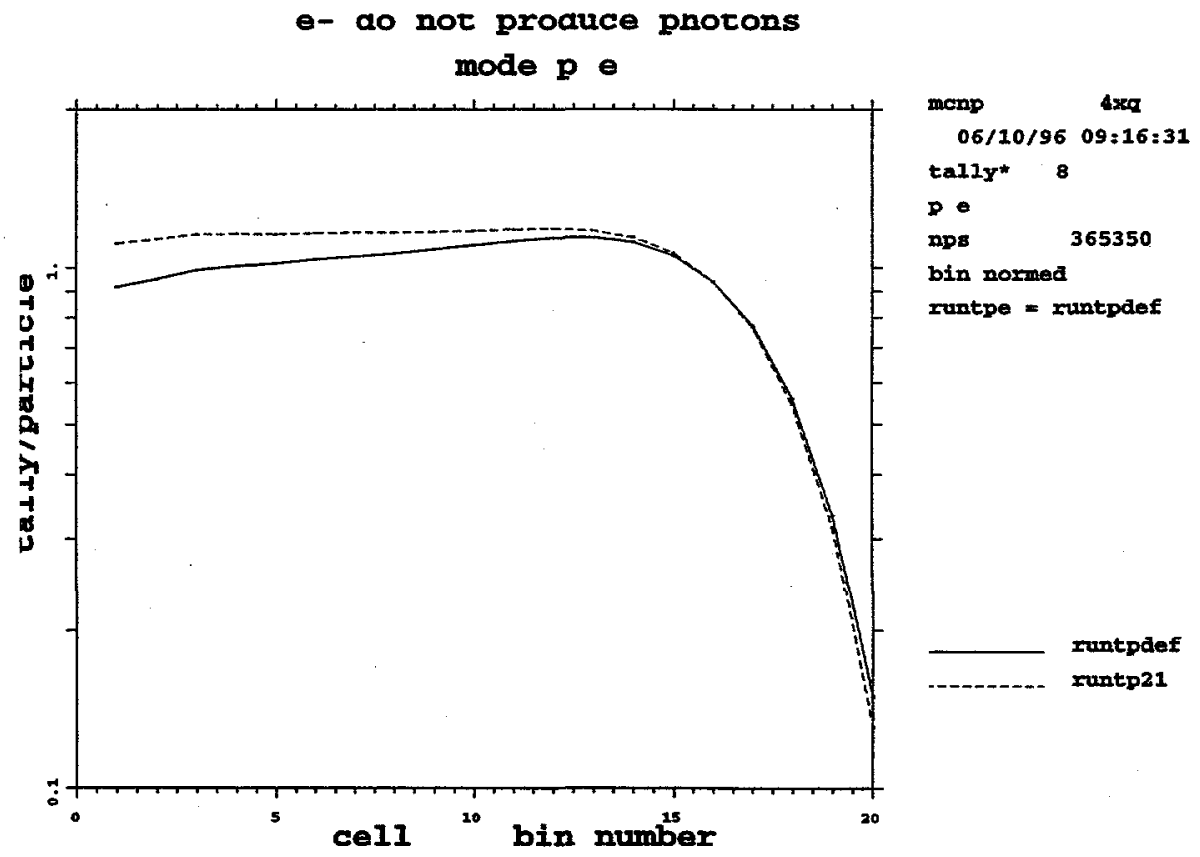

Fig. 65. The solid line indicates default settings. The dashed line indicates the same settings, except that electrons do not produce photons. In both cases, the problem includes photons and electrons. 


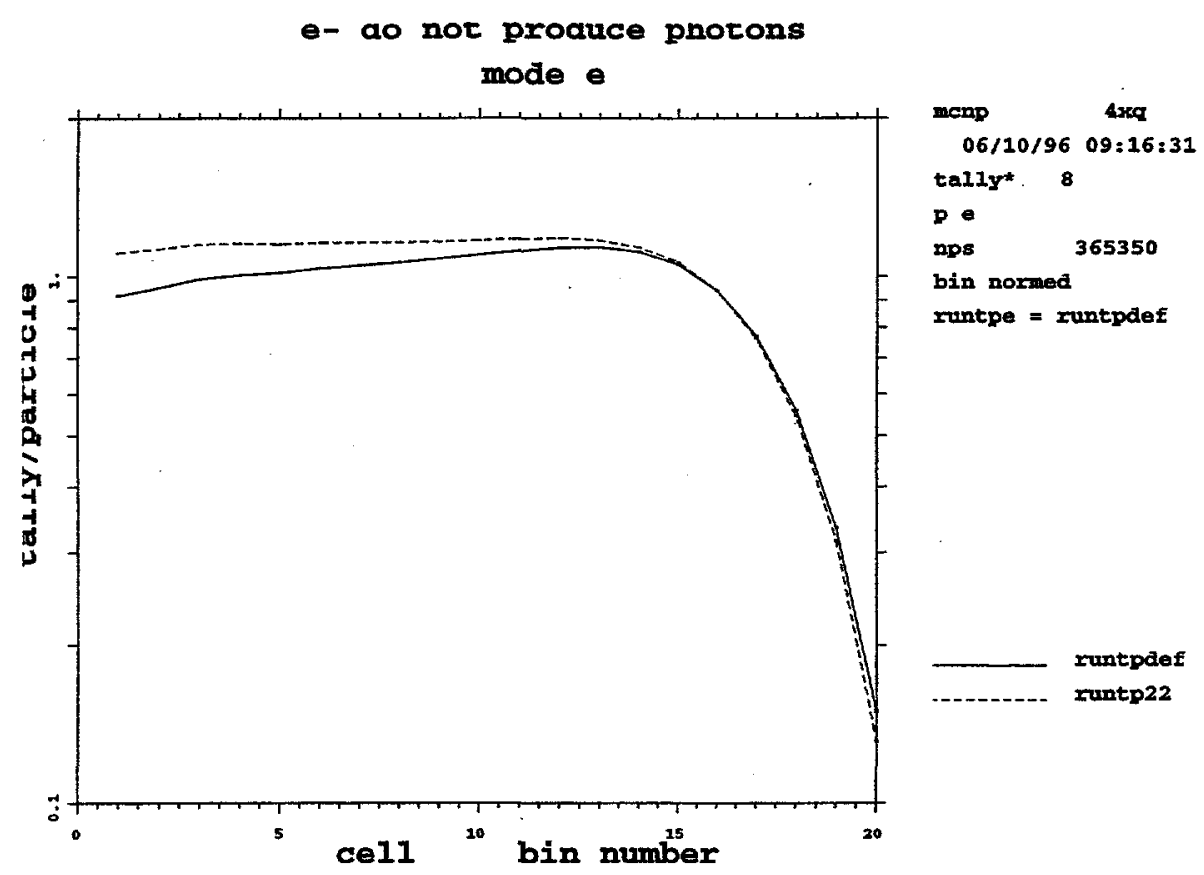

Fig. 66. The solid line indicates default settings. The dashed line indicates the same settings, except that electrons do not produce photons. For the default case, the problem includes photons and electrons, whereas only electrons are included in the other case.

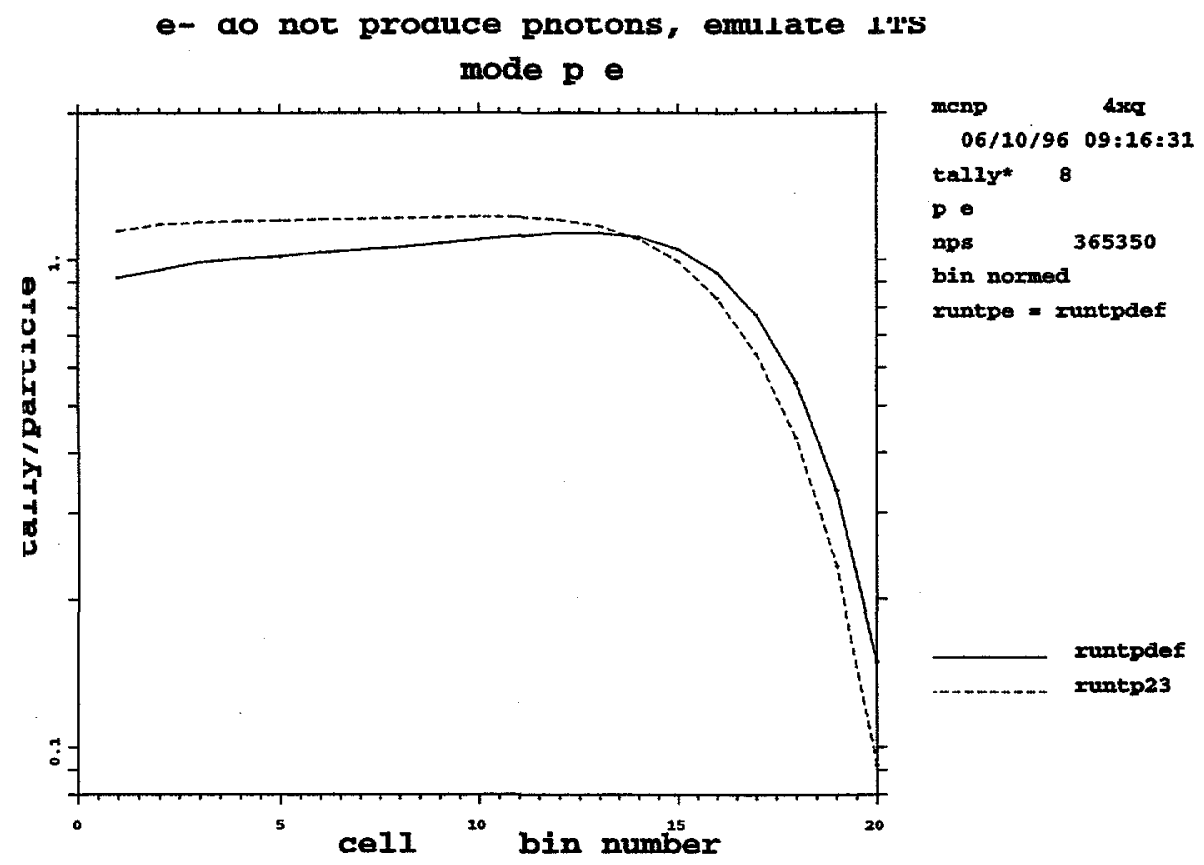

Fig. 67. The solid line indicates default settings. The dashed line indicates the same settings, except that electrons do not produce photons, and the ITS energy grid is used. In both cases, the problem includes photons and electrons. 


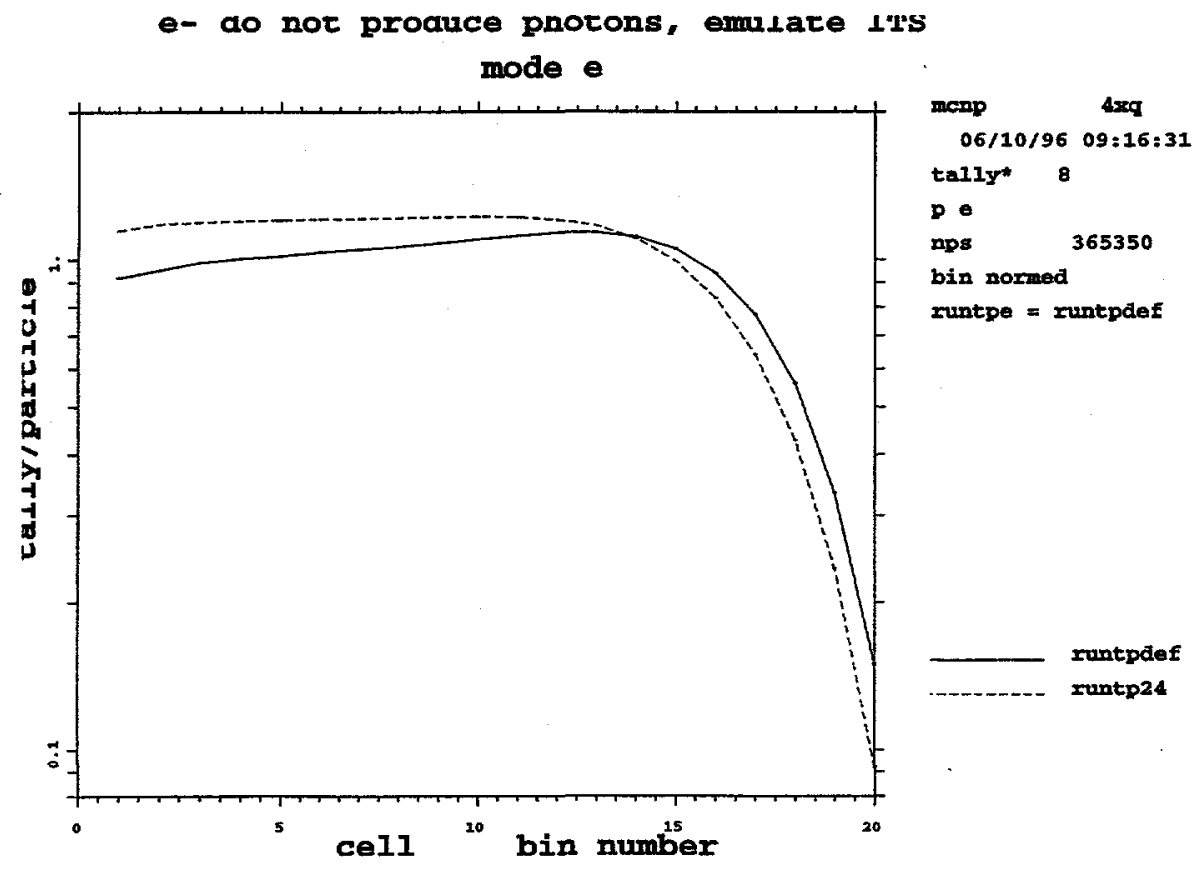

Fig. 68. The solid line indicates default settings. The dashed line indicates the same settings, except that electrons do not produce photons, and the ITS energy grid is used. For the default case, the problem includes photons and electrons, whereas only electrons are included in the other case.

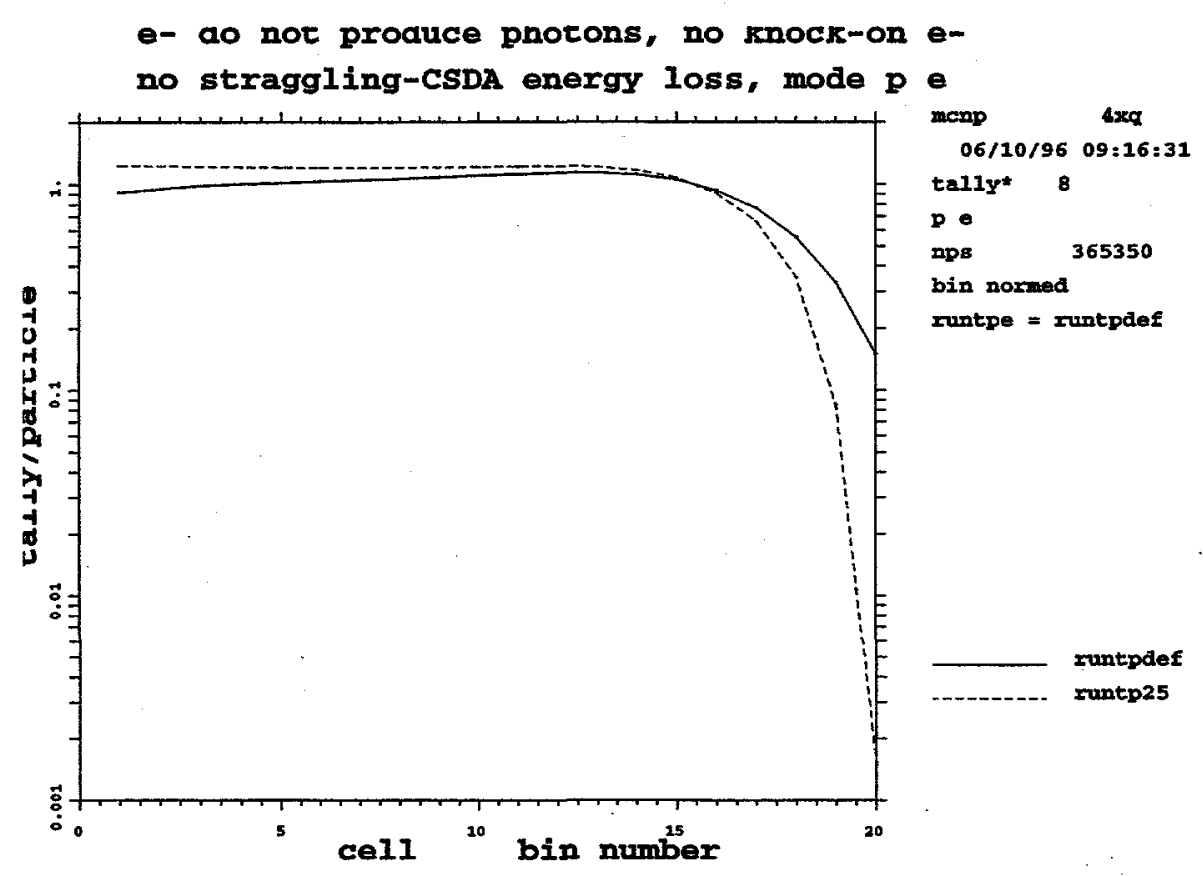

Fig. 69. The solid line indicates default settings. The dashed line indicates the same settings, except that electrons do not produce photons, there are no knock-on electrons, and CSDA energy loss. In both cases, the problem includes photons and electrons. 


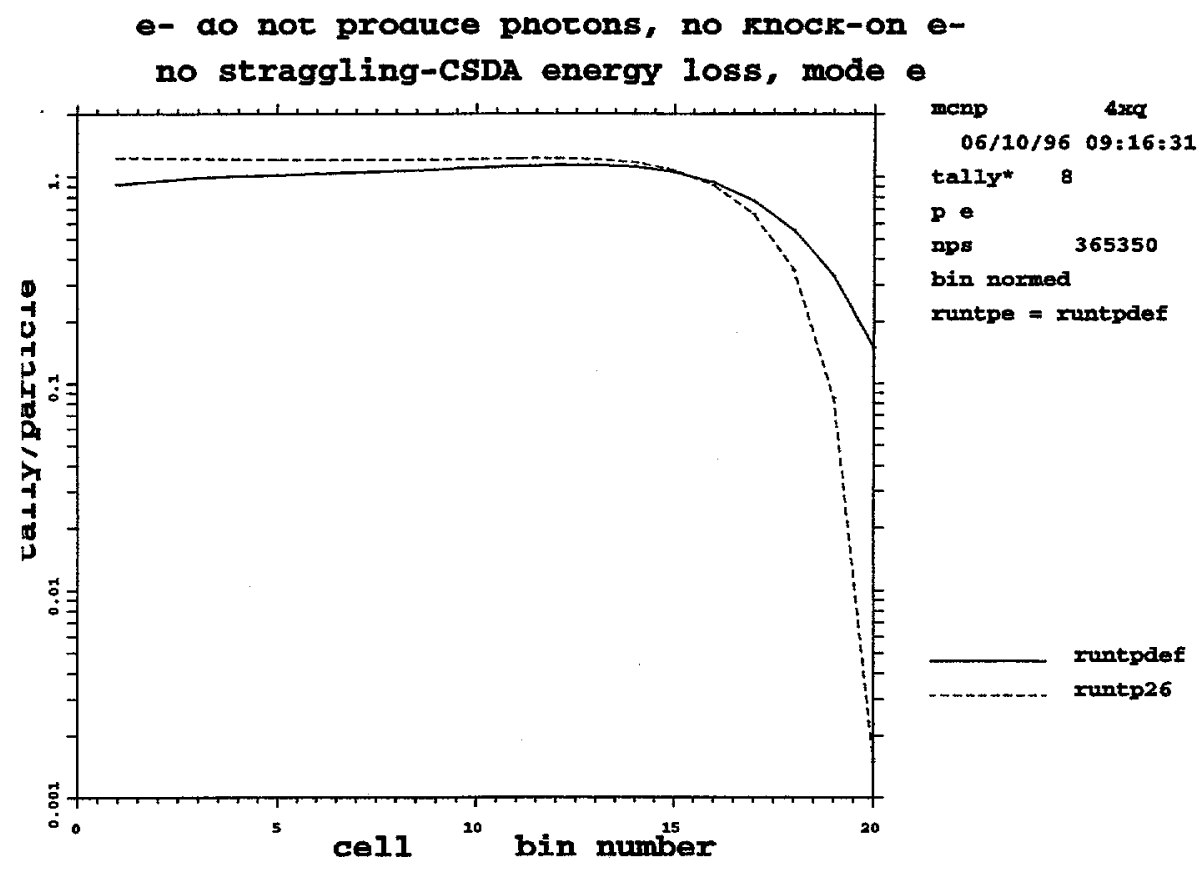

Fig. 70. The solid line indicates default settings. The dashed line indicates the same settings, except that electrons do not produce photons, there are no knock-on electrons, and CSDA energy loss. For the default case, the problem includes photons and electrons, whereas only electrons are included in the other case.

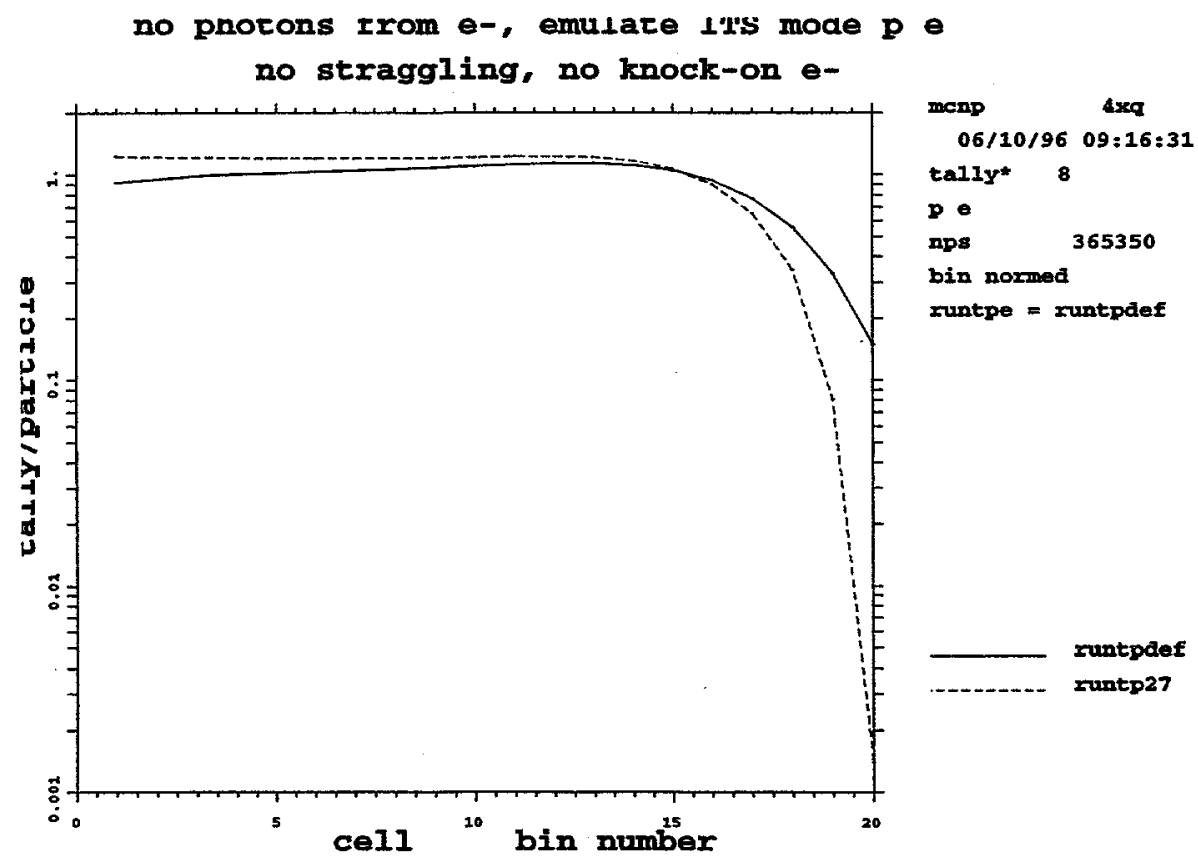

Fig. 71. The solid line indicates default settings. The dashed line indicates that electrons do not produce photons, there are no knock-on electrons, the ITS energy grid is used, and CSDA energy loss. In both cases, the problem includes photons and electrons. 


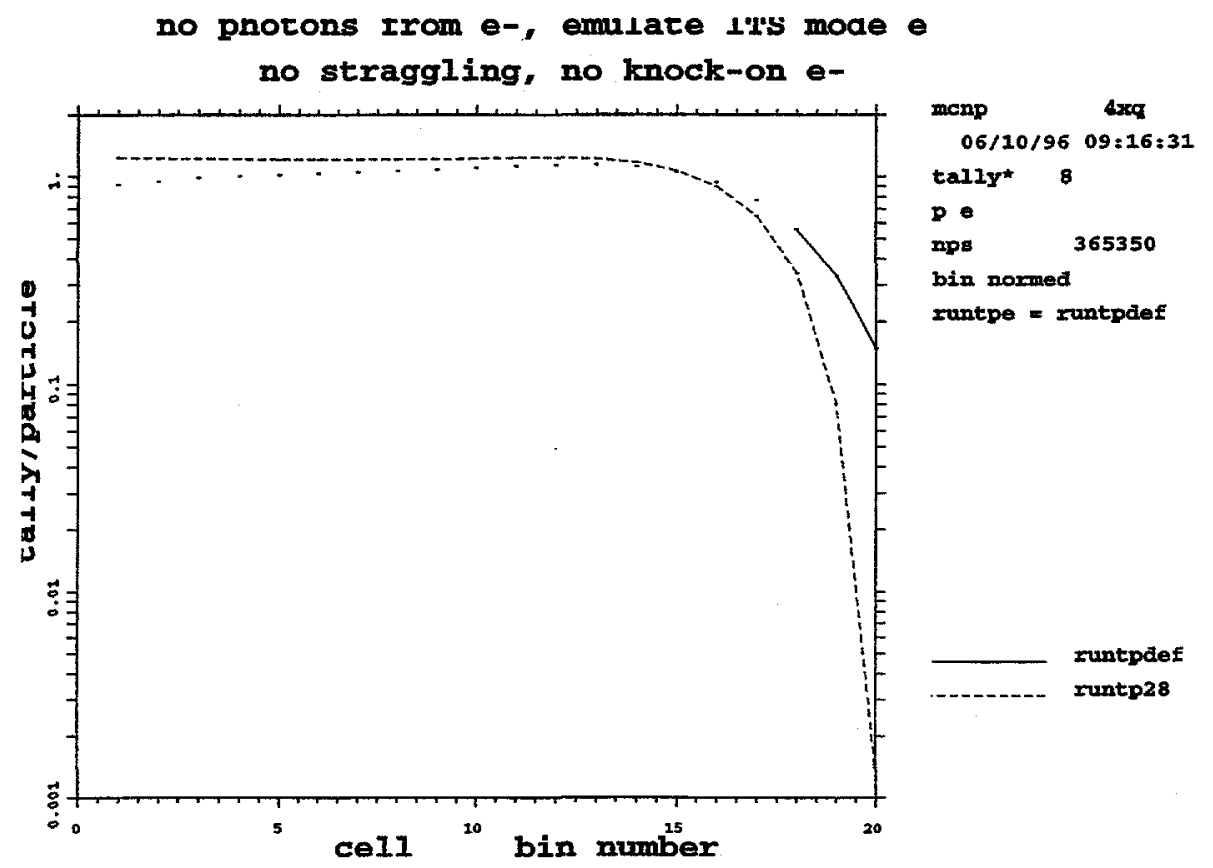

Fig. 72. The solid line indicates default settings. The dashed line indicates that electrons do not produce photons, there are no knock-on electrons, the ITS energy grid is used, and CSDA energy loss. For the default case, the problem includes photons and electrons, whereas only electrons are included in the other case.

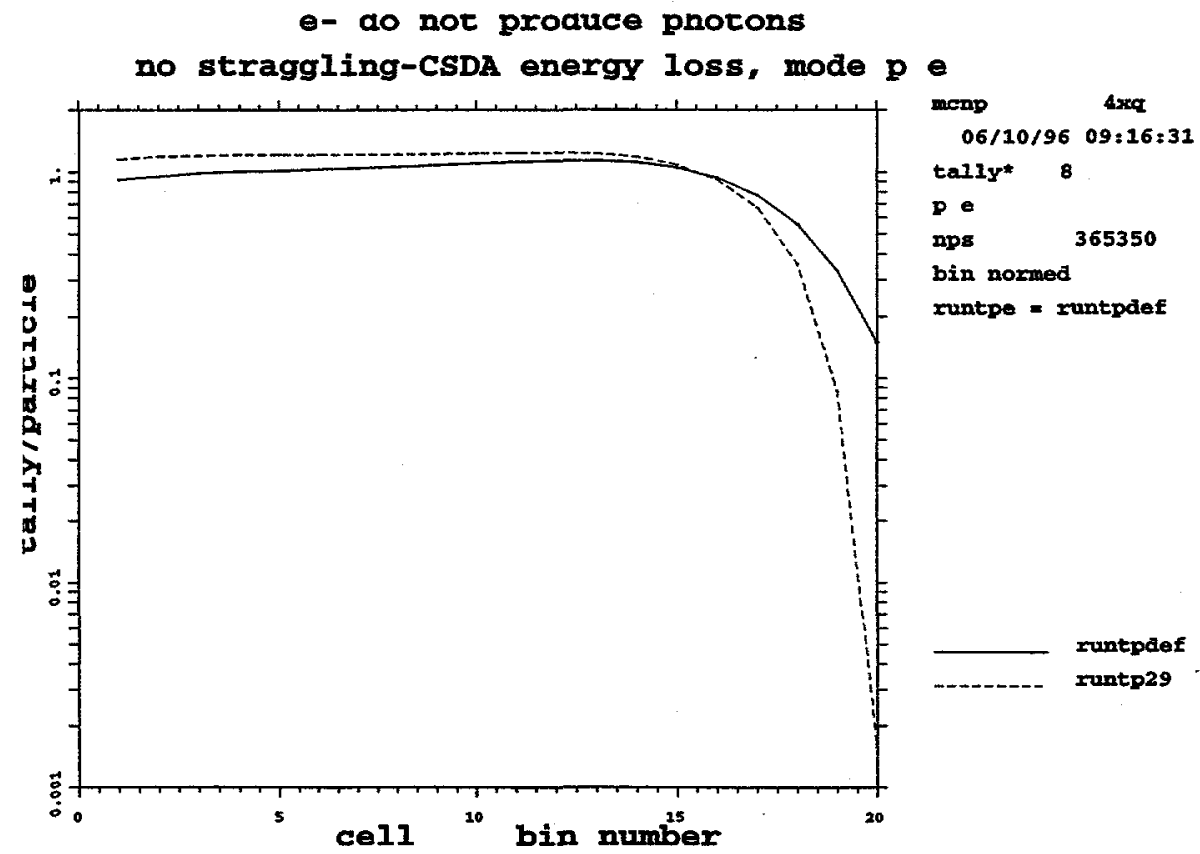

Fig. 73. The solid line indicates default settings. The dashed line indicates the same settings, except that electrons do not produce photons, and there is CSDA energy loss. In both cases, the problem includes photons and electrons. 


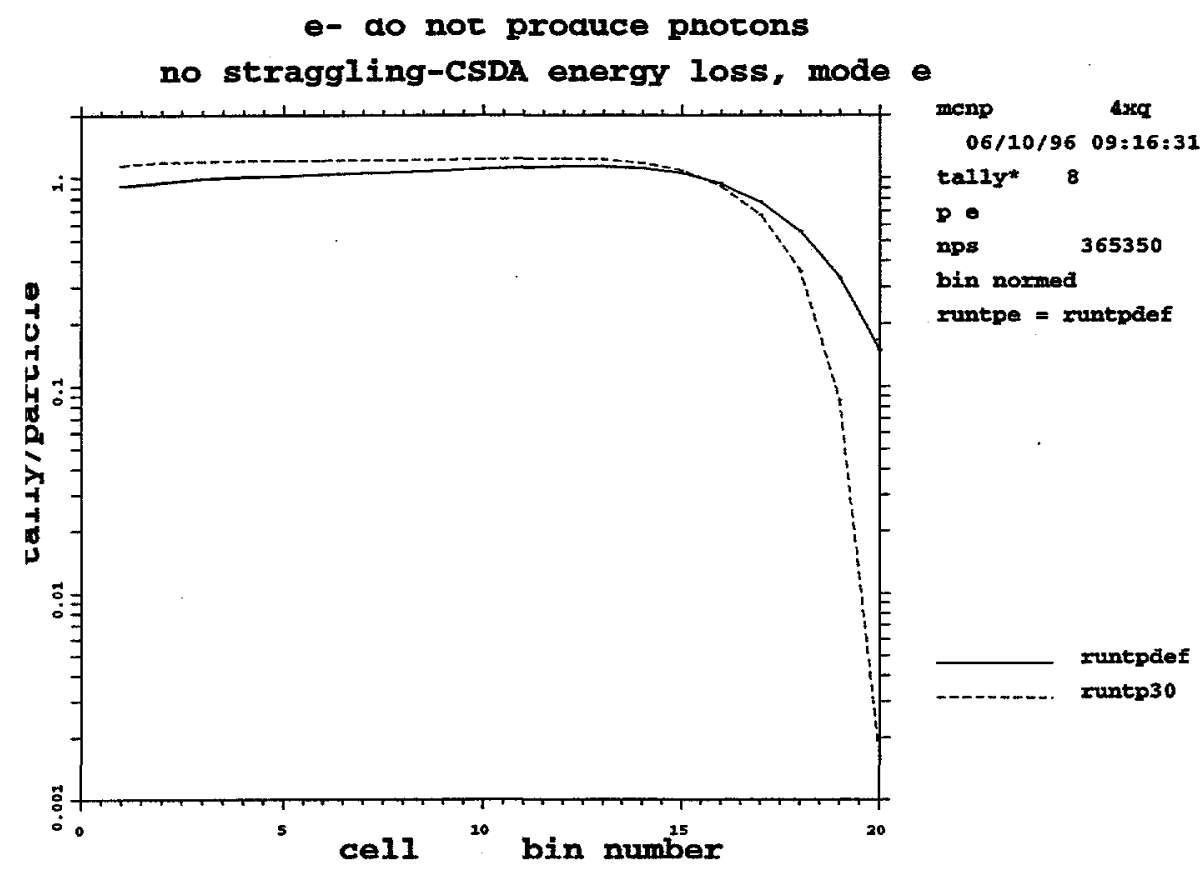

Fig. 74. The solid line indicates default settings. The dashed line indicates the same settings, except that electrons do not produce photons, and there is CSDA energy loss. For the default case, the problem includes photons and electrons, whereas only electrons are included in the other case.

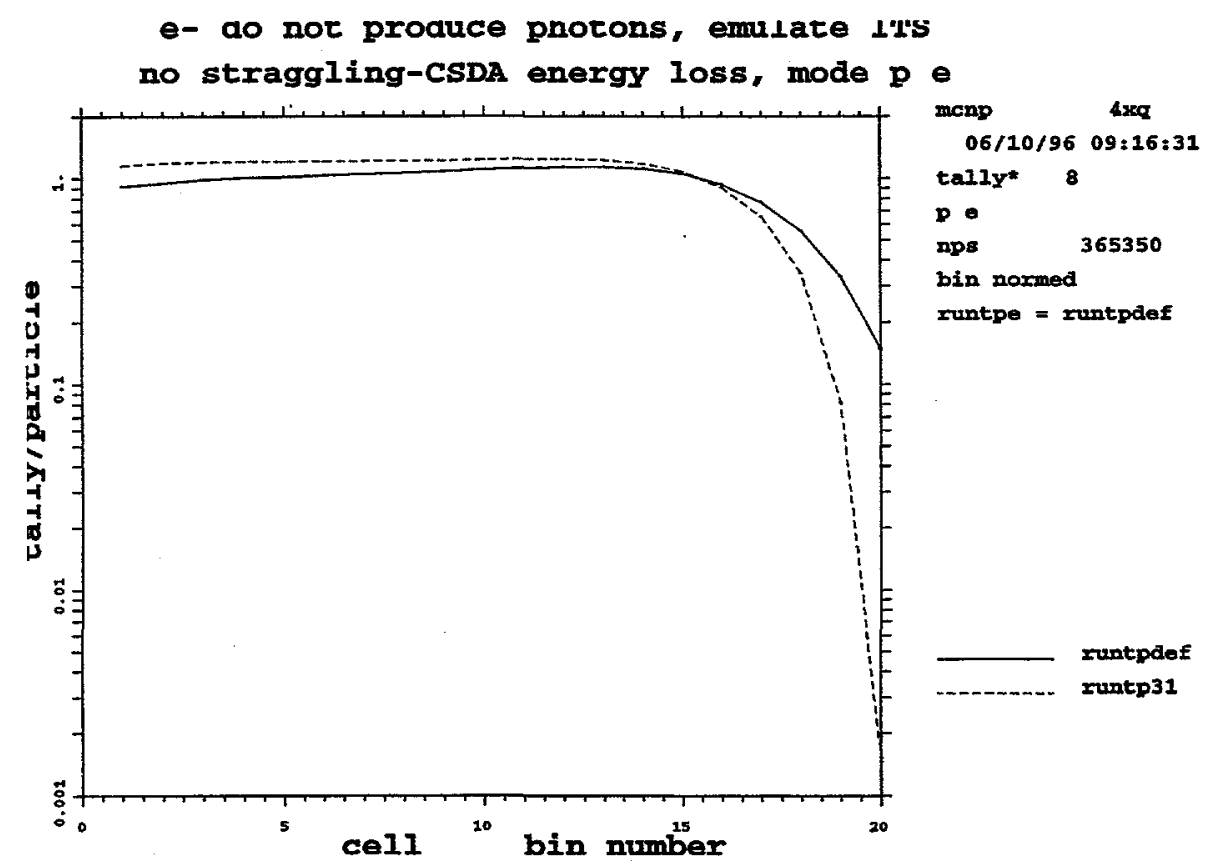

Fig. 75. The solid line indicates default settings. The dashed line indicates the same settings, except that electrons do not produce photons, the ITS energy grid is used, and there is CSDA energy loss. In both cases, the problem includes photons and electrons. 


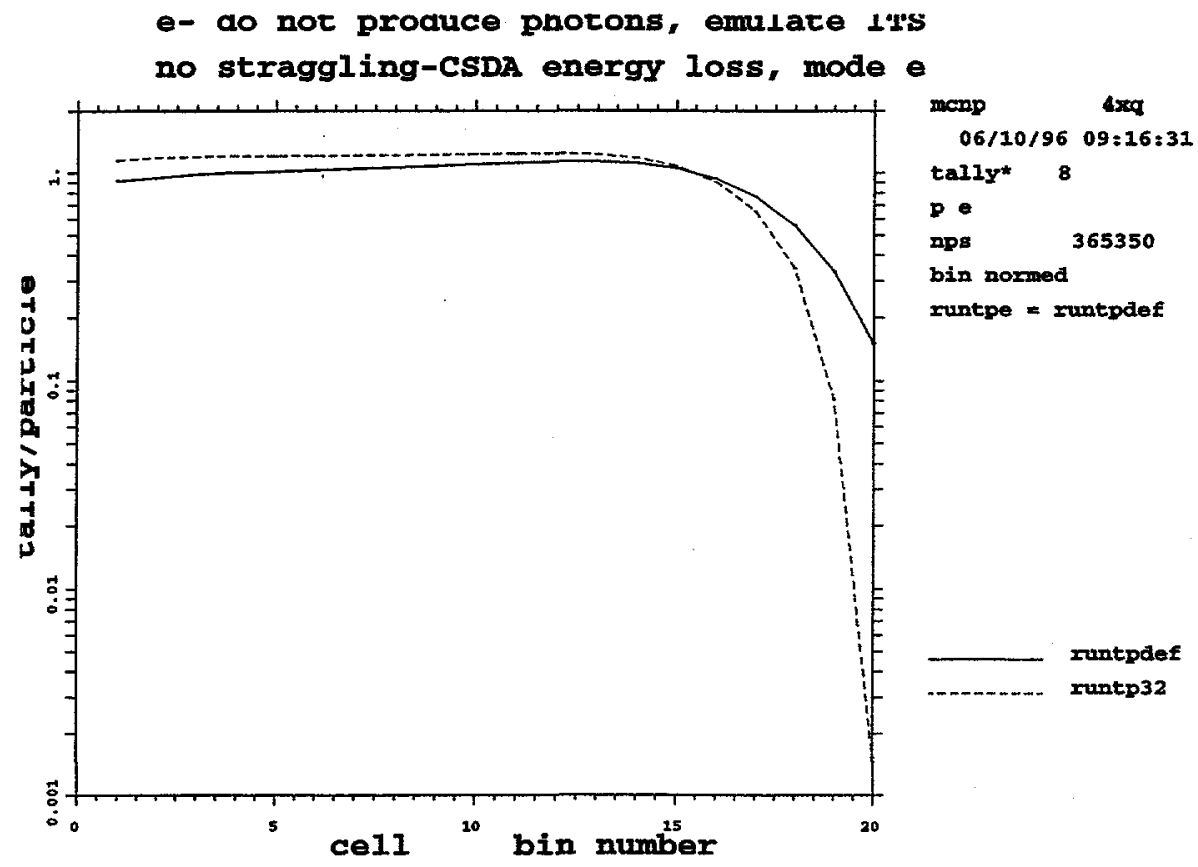

Fig. 76. The solid line indicates default settings. The dashed line indicates the same settings, except that electrons do not produce photons, the ITS energy grid is used, and there is CSDA energy loss. For the default case, the problem includes photons and electrons, whereas only electrons are included in the other case.

\section{CONCLUSIONS}

MCNP4B was verified against a wide range of electron/photon experiments including high-energy bremsstrahlung production and electron transmission and reflection. The bremsstrahlung spectral shape and mean energy compared well across three benchmark experiments. The energy integrated yields agreed within about $10 \%$ for cell tallies and $5 \%$ for detector tallies when compared to the experiments of Faddegon et al., except for a few points near $0^{\circ}$ and $90^{\circ}$. The cell tally energy integrated yields for O'Dell, however, agreed to within $5 \%$ of the experiment and were slightly higher than the experimental results. The calculations of electron transmission based on the experiments of Ebert et al., compare within 5-15\% for silver and uranium, but there are highly significant deviations for carbon. The backscatter verification calculations, for the most part, agree with experiment within $8-20 \%$, with the range of data from several experiment spanning the MCNP results. The overall agreement suggests that electron backscatter calculations in MCNP still warrant improvement. 
Several physics parameters have been shown to affect the results and efficiency of the calculations. The choice of bremsstrahlung angular model was shown to impact the calculated results with cell and detector tallies showing better agreement when the same model is used. The electron sub-step artifacts for bremsstrahlung sampling in MCNP4A were shown to be mitigated in MCNP4B. The energy grid sampling scheme was shown to affect the integrated bremsstrahlung yields at high angles, and significantly impacted the results of electron transmission for carbon, but showed a marginal effect for silver and uranium. The bremsstrahlung splitting scheme presently in MCNP could be enhanced by a modified splitting scheme that has undergone preliminary testing. Furthermore, the effect of including knock-on electrons and secondary photons, as well as varying the energy grid and straggling schemes was studied for a suite of depth dose in water calculations.

This study has also demonstrated the significant improvement in the default performance of MCNP4B compared to MCNP4A. Specifically, the enhancements in secondary particle production algorithms and ESTEP performance, as well as improved stopping powers and energy loss parameters have been demonstrated.

The calculations done using the default settings in MCNP4B show excellent overall agreement with experiment. This study has shown that the default parameters should be modified in a few instances. The electron transmission comparisons in carbon show improved agreement with experiment when using the nearest group energy indexing algorithm (DBCN:18=1) option. It is therefore recommended that the $\mathrm{DBCN}: 18=1$ be used for high energy electrons $(\sim 10 \mathrm{MeV})$ in low $\mathrm{Z}$ materials. Furthermore, after further verification for the full range of experiments and representative target thicknesses in this study, the modified BNUM splitting scheme should be made default in MCNP4C.

There are several areas that can be addressed in further study. The energy indexing algorithms still need to be studied further. The bremsstrahlung angular distributions can be improved, since the energy spectra are already in excellent agreement. More computer time should be invested in the calculations presented here to assess the statistical convergence of the differential energy spectra. Finally, more benchmark studies ${ }^{18}$ should be performed to examine a wider range of experiments. 


\section{REFERENCES}

1. J. F. Briesmeister, "MCNP - A General Monte Carlo N-Particle Transport Code, Version 4B," Los Alamos National Laboratory report, LA-12625-M (1997).

2. W. R. Nelson, H. Hirayama, and D. W. O. Rogers, "The EGS4 code system," SLAC-Report265, Stanford Linear Accelerator Center (Dec. 1985).

3. B. A. Faddegon and D. W. O Rogers, "Comparisons of Thick-Target Bremsstrahlung Calculations by EGS4/PRESTA and ITS version 2.1," Nuc. Inst. Meth. A 327, pp. 556-565 (1993).

4. J. A. Halbleib, R. P. Kensek, T. A. Mehlhorn, G. D. Valdez, S. M. Seltzer, and M. J. Berger, "ITS Version 3.0: The Integrated TIGER Series of Coupled Electron/Photon Monte Carlo Transport Codes," Sandia National Laboratories report SAND91-1634 (March 1992).

5. J. J. DeMarco, T. D. Soldberg, R. E. Wallace, and J. B. Smathers, "A Verification of the Monte Carlo Code MCNP for Thick Target Bremsstrahlung Calculations," Med. Phys. 22, pp. 11-16, (1995).

6. B. A. Faddegon, C. K. Ross, and D. W. O. Rogers, "Forward-Directed Bremsstrahlung of 10- to 30-MeV Electrons Incident on Thick Targets of $\mathrm{Al}$ and Pb," Med. Phys. 17, pp. 773785, (1990).

7. B. A. Faddegon, C. K. Ross, and D. W. O. Rogers, "Angular Distribution of Bremsstrahlung from 15-MeV Electrons Incident on Thick Targets of $\mathrm{Be}, \mathrm{Al}$, and $\mathrm{Pb}$," Med. Phys. 18, pp. 727-739 (1991).

8. A. A. O'Dell, C. W. Sandifer, R. B. Knowlen, and W. D. George, "Measurement of Absolute thick-target Bremsstrahlung Spectra," Nuc. Inst. Meth. 61, pp. 340-346 (1968).

9. N. Starfelt and H. W. Koch, "Differential Cross-Section Measurements of Thin-target Bremsstrahlung Produced by 2.7- to 9.7- MeV Electrons," Phys. Rev. 102, pp.1598-1612 (1956).

10. M. J. Berger and S. M. Seltzer, "Bremsstrahlung and Photoneutrons from Thick Tungsten and Tantalum Targets," Phys. Rev. C 2, pp. 621-631, (1970).

11. P. J. Ebert, A. F. Lauzon, and E. M. Lent, "Transmission and Backscattering of 4.0- to 12.0MeV Electrons," Phys. Rev. 183, pp. $422-430$ (1969).

12. R. W. Dressel, "Retrofugal Electron Flux from Massive Targets Irradiated with a Monoenergetic Primary Beam," Phys. Rev. 144, pp. 332-343 (1966).

13. H.A. Bethe and W. Heitler, "On stopping of fast particles and on the creation of positive electrons," Proc. Roy. Soc. (London) A146, pp. 83 (1934). 
14. D. W. O. Rogers and A. F. Bielajew, "20 MeV Electrons on a Slab of Water," in Monte Carlo Transport of Electron 's and Photons, T. M. Jenkins, W. R. Nelson, and A. Rindi, eds., Plenum Press, 1987.

15. A. E. Nahum, "Overview of Photon and Electron Monte Carlo," in Monte Carlo Transport of Electrons and Photons, T. M. Jenkins, W. R. Nelson, and A. Rindi, eds., Plenum Press (1987).

16. T. Tabata, "Backscattering of Electrons from 3.2 to $14 \mathrm{MeV}$," Phys. Rev. 162 p. 336-347, (1967).

17. H. W. Koch and J. W. Motz, "Bremsstrahlung Cross-Section Formulas and Related Data," Rev. Mod. Phys. 31 p. 920, (1959).

18. C. R. Drumm, "Multi-Dimensional Electron-Photon Transport with Standard Discrete Ordinates codes," Nucl. Sci. Eng. 127, p. 1-21 (1997). 


\section{APPENDIX A}

\section{Modified BNUM Sampling Patch}


This patch is located on cfs in 119238/bench/install.bnum.

*ident,bnum

*d,ny.444

if(nwc.eq.6)then

bnum $=\max$ (zero,ritm)

if(bnum.ne.zero)enum=one/bnum

endif

*d,bb.14

c

c set up to produce none, one, or more photons.

el=zero

npa $=1$

$\mathrm{nb}=1$

c

c do splitting or roulette if bnum biasing is used.

if(bnum.ne.one)then

$\mathrm{nb}=$ bnum+rang 0

if(nb.eq. 0$)$ then

npa $=0$

$\mathrm{nb}=1$

endif

endif

$w b=w g t$

if(bnum.ne.zero)wb=wgt/bnum

$\mathrm{rb}=\mathrm{nb}$

c

c prepare to make a photon and save the stack.

c sample the site and get the electron energy for each possible photon

c and record energy loss, el.

$5 \mathrm{npb}=\mathrm{npb}+1$

*d,bb.21,bb.23

*i,bb. 25

wgt $=w b$

*d,cor4-1.163

if(iphot.ne.0.or.fiml(2).eq.0.or.es.It.elc(2).or.

\& npa.eq.0)go to 200

*d,bb.71,bb.74

*d,cor4-1.167

*i,bb.149

$\mathrm{nb}=\mathrm{nb}-1$

if(nb.gt.0)go to 5

*i,bb.151

$\mathrm{el}=\mathrm{el} / \mathrm{rb}$ 
APPENDIX B

MCNP Input Files 
Input File B.1： Faddegon input files

These input files and associated output files are located on cfs in /x6code/benchmarks/electron/ faddegon/fad.al.tar, fad.be.tar, and fad.pb.tar. This input file is based on the aluminum with stainless steel configuration. The changes needed for lead and beryllium are indicated in italics.

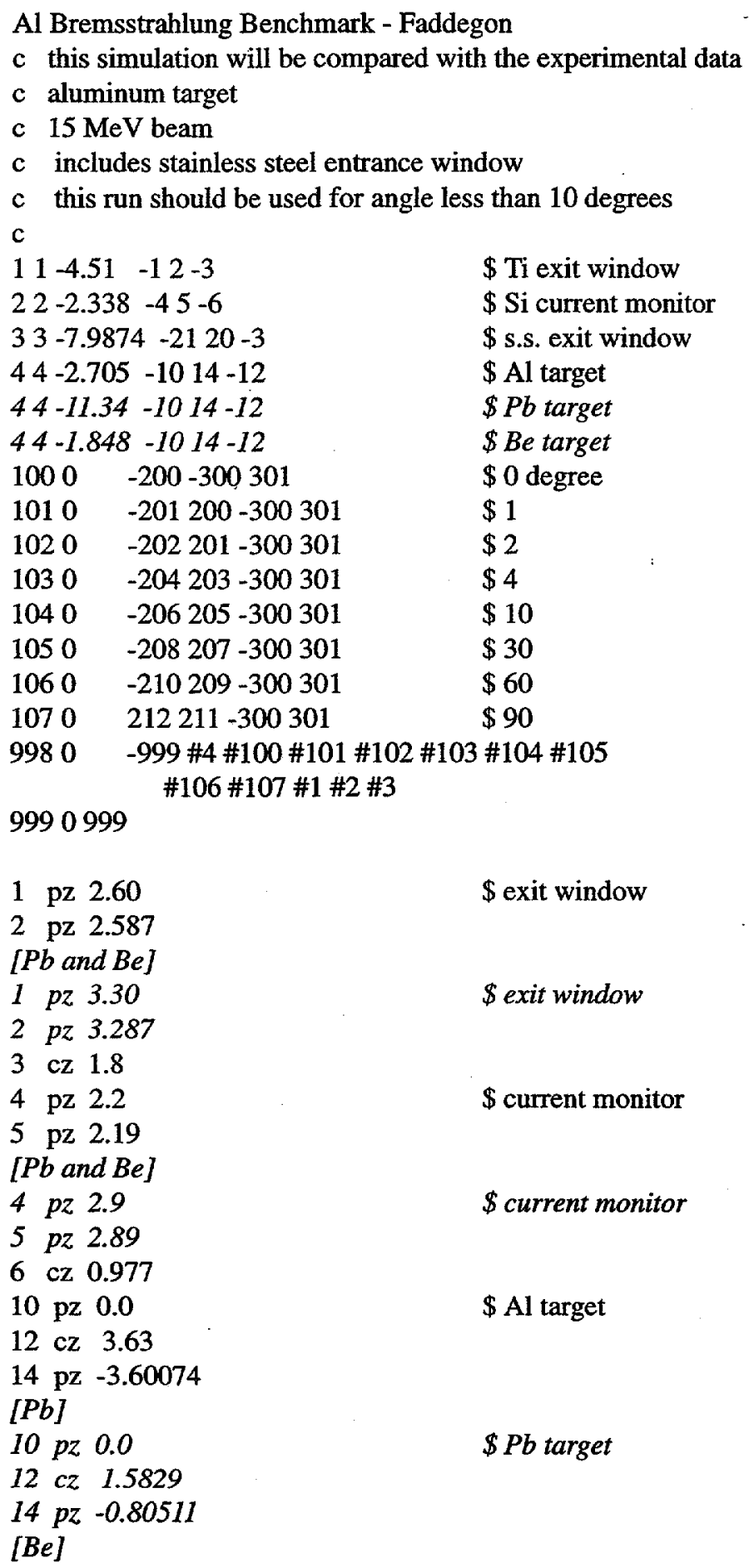


$10 \mathrm{pz} 0.0$

$12 \mathrm{cz} 3.6364$

$14 p z-6.3149$

$20 \mathrm{pz} 0.9$

$21 \mathrm{pz} 0.9051$

$100 \mathrm{pz} 2.601$

[ $\mathrm{Pb}$ and $\mathrm{Be}$ ]

$100 \mathrm{pz} 3.301$

$c$ cones for cell tallies

$200 \mathrm{kz} 0.7 .6158 \mathrm{e}-5$ - $1 \$ 0.5$ degree

$201 \mathrm{kz} 0.6 .8570 \mathrm{e}-4-1 \$ 1.5$

$202 \mathrm{kz} 0.1 .9063 \mathrm{e}-3-1 \$ 2.5$

$203 \mathrm{kz} 0.3 .7409 \mathrm{e}-3-1 \$ 3.5$

$204 \mathrm{kz} 0.6 .1940 \mathrm{e}-3 \mathrm{-1} \$ 4.5$

$205 \mathrm{kz} 0.2 .8004 \mathrm{e}-2$-1 $\$ 9.5$

$206 \mathrm{kz} 0.3 .4351 \mathrm{e}-2-1 \quad \$ 10.5$

$207 \mathrm{kz} 0.3 .2010 \mathrm{e}-1$-1 $\$ 29.5$

$208 \mathrm{kz} 0.3 .4697 \mathrm{e}-1-1 \quad \$ 30.5$

$209 \mathrm{kz} 0.2 .8821 \mathrm{e} 0-1 \$ 59.5$

$210 \mathrm{kz} 0.3 .1240 \mathrm{e} 0-1 \$ 60.5$

$211 \mathrm{kz} 0.1 .3131 \mathrm{e} 4-1 \$ 89.5$

$212 \mathrm{kz} 0.1 .3131 \mathrm{e} 4 \quad 1 \quad \$ 90.5$

c spheres for cell tallies ( $1 \mathrm{~mm}$ radial thickness)

300 so 300 .

301 so 299.9

c outside world

999 so 500

mode p e

imp:p 1 12r 0

imp:e $112 \mathrm{r} 0$

cut:p j 0.145 3j

cut:e j $0.1453 \mathrm{j}$

phys:e $15.8 \mathrm{j}$

sdef par=3 sur=100 pos=0 02.601 vec=0 $0-1$ dir=1 rad=d1 erg=15.

$[\mathrm{Pb}$ and $\mathrm{Be}]$

sdef par $=3$ sur $=100$ pos $=003.301$ vec $=00-1$ dir $=1 \mathrm{rad}=d 1$ erg $=15$.

sil 0.05

c

c note: fm tally multipliers convert to per steradian

c by multiplyting by $(300 \mathrm{~cm})^{\wedge} 2$

$c$ this is the source to detector distance

$c$ the energy spectra, to agree with Faddegon, must be

$c$ divided by the width of the energy bin

c

c cell tallies

f4:p $1006 \mathrm{i} 107$

fm4 9.e4 \$ convert to per steradian

fq4 ef

c

c ring detectors

$\mathrm{c}$ the first and last tally is offset slightly since detectors

$c$ located right on a surface cause trouble

fc5 0 degree
$\$$ Be target

\$ stainless steel window

$\$$ surface for starting source

$\$$ surface for starting source 


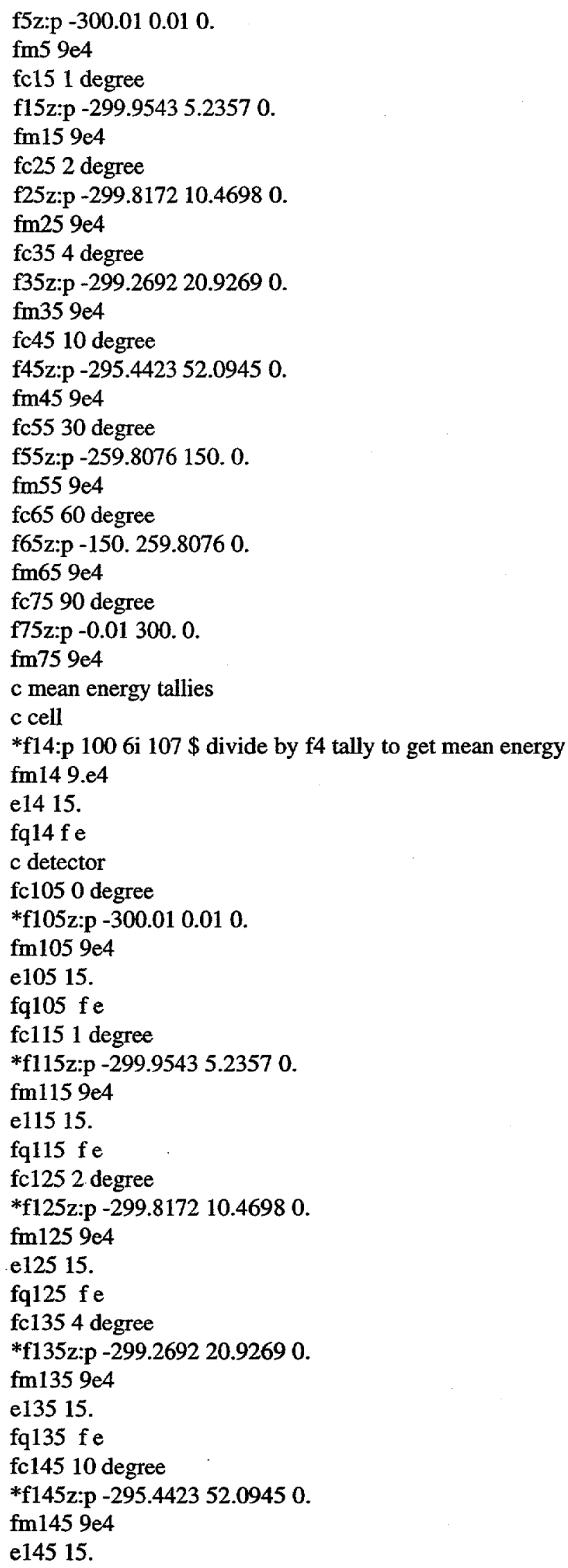




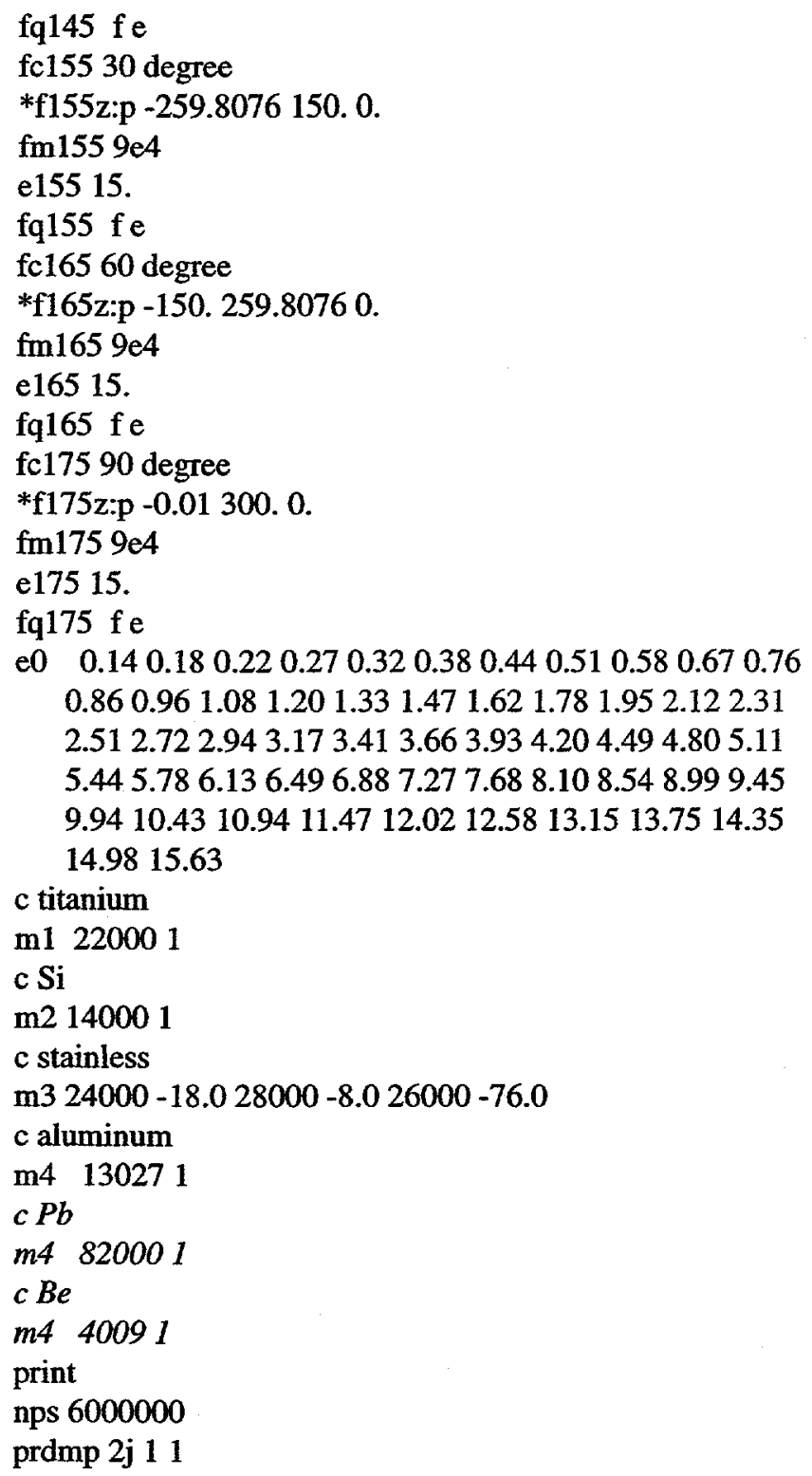


Input File B.2: Faddegon, Aluminum target, no stainless steel entrance window

This input file is based on the aluminum without stainless steel configuration. The changes needed for lead and beryllium are indicated in italics.

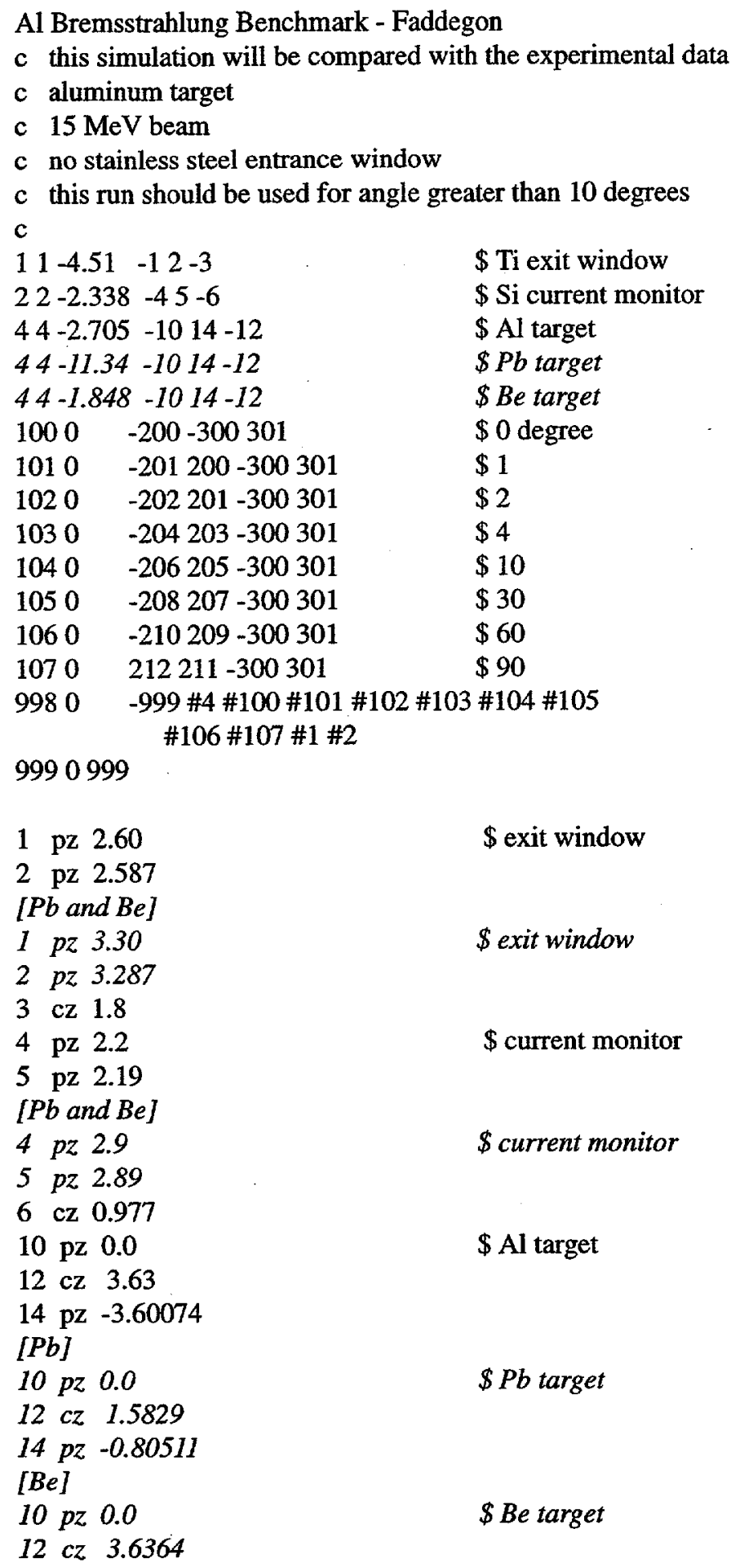


c cones for cell tallies

$200 \mathrm{kz} 0.7 .6158 \mathrm{e}-5-1 \$ 0.5$ degree

$201 \mathrm{kz} 0.6 .8570 \mathrm{e}-4-1 \$ 1.5$

$202 \mathrm{kz} 0.1 .9063 \mathrm{e}-3-1 \$ 2.5$

$203 \mathrm{kz} 0.3 .7409 \mathrm{e}-3-1 \$ 3.5$

$204 \mathrm{kz} 0.6 .1940 \mathrm{e}-3-1 \$ 4.5$

$205 \mathrm{kz} 0.2 .8004 \mathrm{e}-2-1 \$ 9.5$

$206 \mathrm{kz} 0.3 .4351 \mathrm{e}-2-1 \$ 10.5$

$207 \mathrm{kz} 0.3 .2010 \mathrm{e}-1-1 \$ 29.5$

$208 \mathrm{kz} 0.3 .4697 \mathrm{e}-1-1 \$ 30.5$

$209 \mathrm{kz} 0.2 .8821 \mathrm{e} 0-1 \$ 59.5$

$210 \mathrm{kz} 0.3 .1240 \mathrm{e} 0-1 \$ 60.5$

$211 \mathrm{kz} 0.1 .3131 \mathrm{e} 4-1 \$ 89.5$

$212 \mathrm{kz} 0.1 .3131 \mathrm{e} 4 \quad 1 \$ 90.5$

$\mathrm{c}$ spheres for cell tallies ( $1 \mathrm{~mm}$ radial thickness)

300 so 300 .

301 so 299.9

c outside world

999 so 500

mode $\mathrm{p}$ e

imp:p $111 \mathrm{r} 0$

imp:e $111 \mathrm{r} 0$

cut:p j $0.1453 \mathrm{j}$

cut:e $\mathrm{j} 0.1453 \mathrm{j}$

phys:e $15.8 \mathrm{j}$

sdef par $=3$ sur=100 pos $=002.601$ vec=0 $0-1 \mathrm{dir}=1 \mathrm{rad}=\mathrm{d} 1 \mathrm{erg}=15$.

$[\mathrm{Pb}$ and $\mathrm{Be}]$

sdef par $=3$ sur=100 pos $=003.301$ vec $=00-1$ dir $=1$ rad $=d 1$ erg $=15$.

sil 0.05

c

c note: fm tally multipliers convert to per steradian

c by multiplyting by $(300 \mathrm{~cm})^{\wedge} 2$

$c$ this is the source to detector distance

c the energy spectra, to agree with Faddegon, must be

$c$ divided by the width of the energy bin

c

c cell tallies

f4:p 1006 i 107

fm4 9.e4 \$ convert to per steradian

fq4 $\mathbf{e} f$

$c$

c ring detectors

$c$ the first and last tally is offset slightly since detectors

$c$ located right on a surface cause trouble

fc5 0 degree

f5z:p -300.010 .010 .

fm5 $9 \mathrm{e} 4$

fc15 1 degree

f15z:p -299.9543 5.2357 0. 


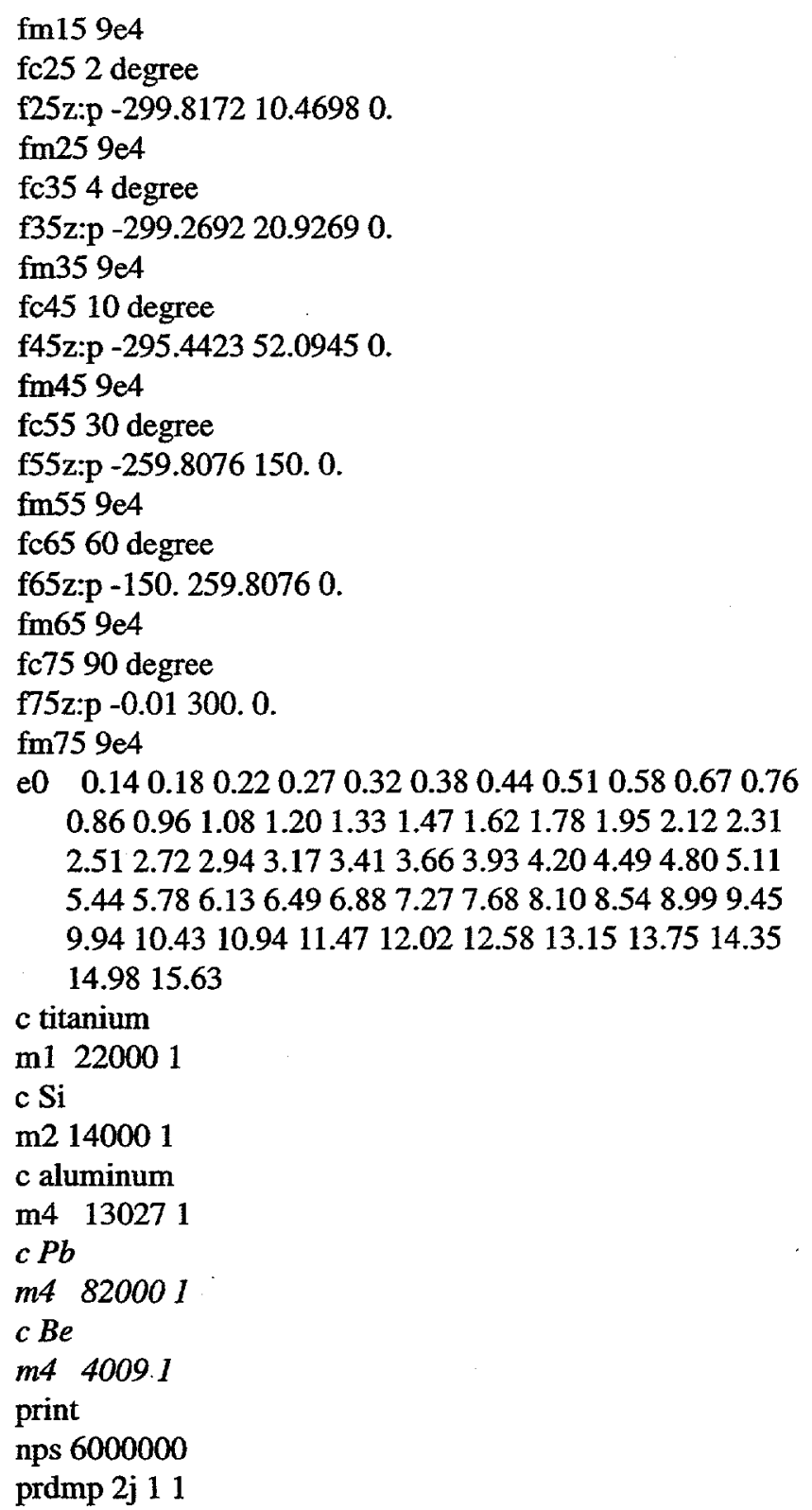




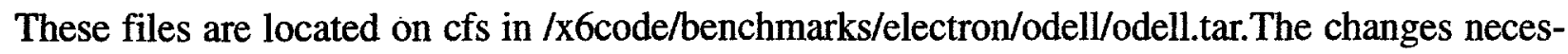
sary for $20.9 \mathrm{MeV}$ electrons are indicated in italics.

$\mathrm{Au} / \mathrm{W}$ Bremsstrahlung Benchmark - O'Dell

c this simulation will be compared with the experimental data

c $\mathrm{Au} / \mathrm{W}$ target

c $10 \mathrm{MeV}$ beam

c

$11-19.24-1014-12$

$22-19.32 \quad 20-14-12$

$1000 \quad-3035-3132-3334$

$9980 \quad-999 \# 1$ \#2 \#100

9990999

$10 \mathrm{pz} 0.0$

$12 \mathrm{cz} 2.6$

$14 \mathrm{pz}-0.0254678$

$20 \mathrm{pz}-0.0381489$

\$ W target

\$ Au target

$\$ 0$ degree tally

$30 \mathrm{pz}-25.6$

31 py 1.905

32 py -1.905

[20.9 MeV]

31 py 0.635

32 py -0.635

$33 \mathrm{px} 0.3175$

$34 \mathrm{px}-0.3175$

$35 \mathrm{pz}-25.7$

$100 \mathrm{pz} 1.0$

\$ W target

$\$$ W thickness of $0.49 \mathrm{~g} / \mathrm{cm} 2$

$\$$ Au thickness of $0.245 \mathrm{~g} / \mathrm{cm} 2$

c outside world

$\$ 1.5$ in face of secondary target

999 so 200

mode $\mathrm{pe}$

imp:p,e $13 r 0$

$c$ these high cutoffs are the same as in experiment

cut:p j 4. $3 \mathrm{j}$

cut:e j $4.3 \mathrm{j}$

$c$ change this card when altering source energy

phys:e 10. 8j

[20.9] phys:e $20.98 j$

sdef par $=3$ sur= 100 pos $=001.0$ vec=0 $0-1 \mathrm{dir}=1 \mathrm{rad}=\mathrm{d} 1 \mathrm{erg}=10$.

[20.9] sdef par $=3$ sur $=100$ pos $=001.0$ vec $=00-1$ dir $=1$ rad $=d 1$ erg $=20.9$

si1 0.1

c

c note: fm tally multipliers convert to per steradian

$c$ by multiplyting by $(25.6 \mathrm{~cm})^{\wedge} 2$

$\mathrm{c}$ this is the source to detector distance

c

$c$ cells

fc4 cell tally - forward direction

f4:p 100 
$\mathrm{fm} 4655.36$

fq4 $\mathrm{e} f$

c detectors

$c$ use point detector for 0 degrees only as a check

fc5 0 degree

f5:p $00-25.50$.

fm5 655.36

c

e0 $\quad 4.04 .24 .44 .64 .85 .05 .25 .45 .65 .86 .0$

6.26 .46 .66 .87 .07 .27 .47 .67 .88 .08 .4

8.89 .29 .610 .

[20.9]

eO 4.0 4.2 4.44.6 4.8 5.0 5.2 5.4 5.6 5.86.0

6.26 .46 .66 .87 .07 .27 .47 .67 .88 .08 .4

8.89 .29 .610 .10 .410 .812 .212 .613 .013 .4

13.814 .214 .615 .015 .415 .816 .216 .617 .0

17.518 .018 .519 .019 .520 .220 .9

$c$ tungsten

m1 740001

$c$ gold

m2 791971

print

nps 4000000

prdmp 2j 11 
These files are located on cfs in $/ x 6$ code/benchmarks/electron/starfelt/star.tar.

W Bremsstrahlung Benchmark - Starfelt and Koch

c this simulation will be compared with the experimental data

c W target $5.8 \mathrm{~g} / \mathrm{cm} 2$ thickness

c $9.66 \mathrm{MeV}$ beam

c

$11-19.24-1014-12$

$1000 \quad-200-300301$

$1040-220210-300301$

$\$$ W target

$\$ 0$ degree

$9980-999 \# 1 \# 100 \# 104$

$\$ 12$

9990999

$10 \mathrm{pz} 0.0$

$12 \mathrm{cz} 2.6$

$14 \mathrm{pz}-0.301455$

$100 \mathrm{pz} 1.0$

$200 \mathrm{kz} \mathrm{0.1.49277e-4-1}$

$210 \mathrm{kz} 0.4 .13928 \mathrm{e}-2-1$

$220 \mathrm{kz} 0.4 .91485 \mathrm{e}-2 \mathrm{-1}$

c spheres for cell tallies

\$ W target

$\$$ corresponds to a $5.8 \mathrm{~g} / \mathrm{cm} 2$ thickness

$\$$ surface for starting source

$\$ 0.7$ degree

$\$ 11.5$

$\$ 12.5$

300 so 120.

301 so 119.7

c outside world

999 so 200

mode $p e$

imp:p,e $13 \mathrm{r} 0$

cut:p j $0.43 \mathrm{j}$

cut:e j $0.43 \mathrm{j}$

c change this card when altering source energy

phys:e $10.4 \mathrm{j} 202 \mathrm{j} 0.05 \mathrm{j}$

sdef $p a r=3$ sur $=100$ pos $=001.0$ vec $=00-1 \mathrm{dir}=1 \mathrm{rad}=\mathrm{d} 1 \mathrm{erg}=9.66$

sil 0.3

c

c note: fm tally multipliers convert to per steradian

c by multiplyting by $(120 \mathrm{~cm})^{\wedge} 2$

$\mathrm{c}$ this is the source to detector distance

c

c cells

fc 4 cell tallies: 0 and 12 degrees

f4:p 100104

fm4 1.44e4

fq4 e f

c detectors

$\mathrm{c}$ use point detector for $\mathbf{0}$ degrees

fc5 0 degree

f5:p $00-120.10$.

fm5 $1.44 \mathrm{e} 4$

c 
c ring detector

fc15 12 degree

f15z:p - 117.3777 24.94940 .

fm15 1.44e4

c

e0 $\quad 0.40 .580 .760 .941 .121 .31 .481 .661 .842 .022 .22 .38$

2.562 .742 .923 .13 .283 .463 .643 .824 .04 .254 .54 .75

5.05.45.8 6.2 6.67.07.5 8.0 8.5 9.09.66

m1 740001

print

nps 15000000

prdmp 2j 11 
Input File B.5: Sample input template for Ebert, transmission and backscatter

These input files and associated output files are located on cfs in /x6code/benchmarks/electron/ ebert/ebert.ag.tar,ebert.c.tar,ebert.u.tar, and ebert.back.tar. This input file is based on silver simulations. The changes needed for carbon and uranium are indicated in italics.

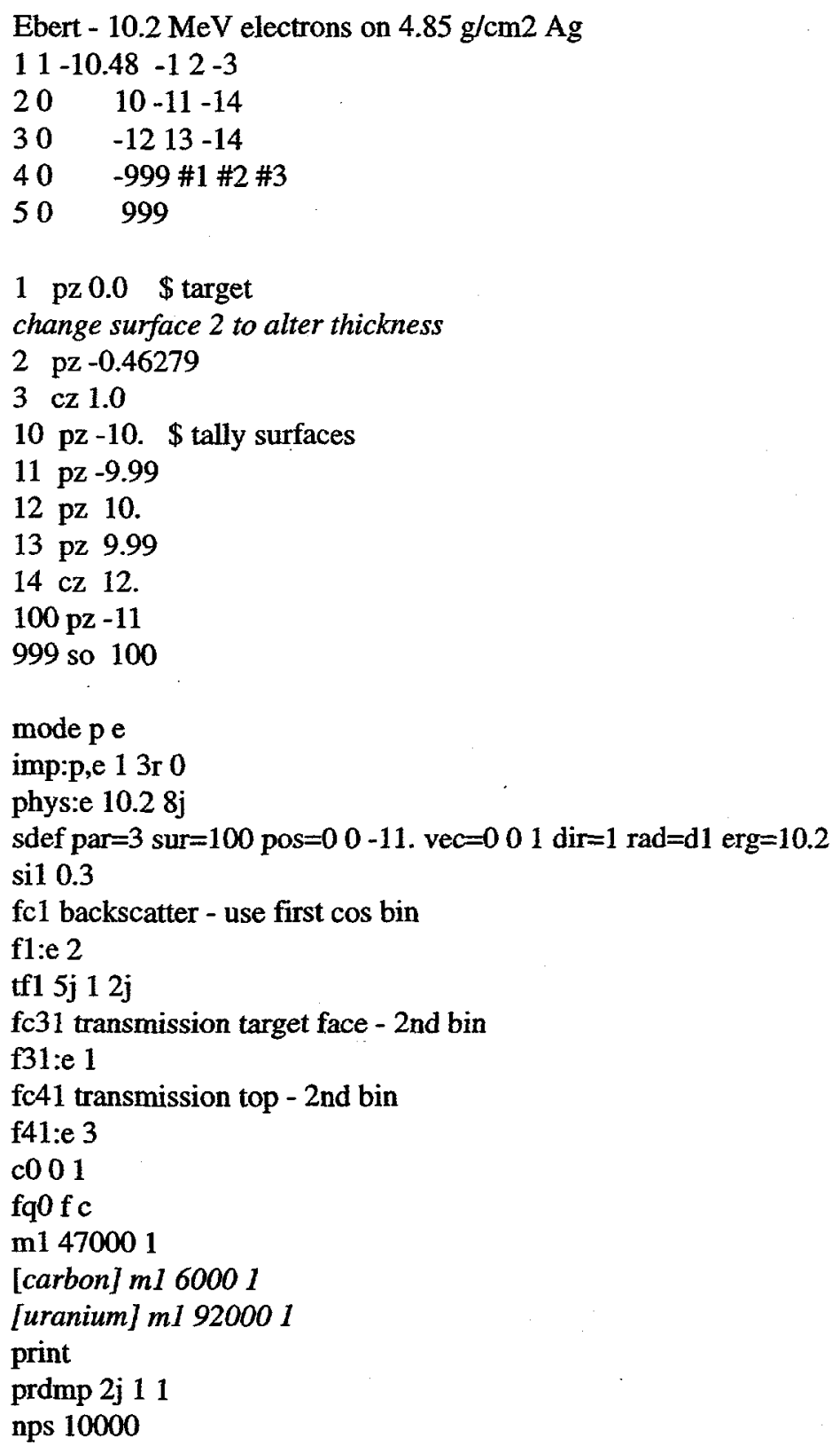


Input File B.6: Input template for $20 \mathrm{MeV}$ electrons in water

These files are located on cfs node / 66 code/benchmarks/electron/water. This is a master input file which highlights the parameters that were changed in italics.

Energy deposition by $20 \mathrm{MeV}$ electrons in water.

$\begin{array}{llllll}1 & 1 & -1 . & 100 & -105 & -300\end{array}$

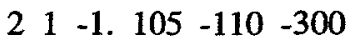

$\begin{array}{llllll}3 & 1 & -1 & 110 & -115 & -300\end{array}$

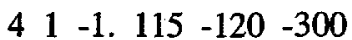

$\begin{array}{llllll}5 & 1 & -1 & 120 & -125 & -300\end{array}$

$\begin{array}{llllll}6 & 1 & -1 & 125 & -130 & -300\end{array}$

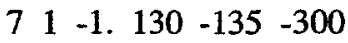

$\begin{array}{lllllll}8 & 1 & -1 & 135 & -140 & -300\end{array}$

$9 \begin{array}{llllll}9 & 1 & -1 . & 140 & -145 & -300\end{array}$

$\begin{array}{lllllll}10 & 1 & -1 . & 145 & -150 & -300\end{array}$

$\begin{array}{llllll}11 & 1 & -1 . & 150 & -155 & -300\end{array}$

$\begin{array}{llllll}12 & 1 & -1 & 155 & -160 & -300\end{array}$

$\begin{array}{llllll}13 & 1 & -1 . & 160 & -165 & -300\end{array}$

$\begin{array}{llllll}14 & 1 & -1 . & 165 & -170 & -300\end{array}$

$\begin{array}{llllll}15 & 1 & -1 . & 170 & -175 & -300\end{array}$

$\begin{array}{llllll}16 & 1 & -1 . & 175 & -180 & -300\end{array}$

$\begin{array}{llllll}17 & 1 & -1 . & 180 & -185 & -300\end{array}$

$\begin{array}{lllllll}18 & 1 & -1 . & 185 & -190 & -300\end{array}$

$\begin{array}{llllll}19 & 1 & -1 . & 190 & -195 & -300\end{array}$

$\begin{array}{lllllll}20 & 1 & -1 & 195 & -200 & -300\end{array}$

$210 \quad(-100: 200: 300)$

$100 \mathrm{px} \quad 0.0$

$105 \mathrm{px} 0.5$

$110 \mathrm{px} 1.0$

$115 \mathrm{px} 1.5$

$120 \mathrm{px} 2.0$

125 px 2.5

$130 \mathrm{px} 3.0$

$135 \mathrm{px} 3.5$

$140 \mathrm{px} 4.0$

$145 \mathrm{px} 4.5$

$150 \mathrm{px} 5.0$

155 px 5.5

$160 \mathrm{px} 6.0$

$165 \mathrm{px} 6.5$

$170 \mathrm{px} 7.0$

$175 \mathrm{px} 7.5$

$180 \mathrm{px} 8.0$

185 px 8.5

$190 \mathrm{px} 9.0$

195 px 9.5

200 px 10.0

300 so 100 
phys:e 20. j iphot $[0,1] \mathrm{j}$ istrg[0,1] $2 \mathrm{j}$ rnok $[0,1] \mathrm{j}$

dbcn 17j dbcn(18)[0,1] \$ Emulate ITS 3. (default=0)

sdef $\operatorname{par}=3$ pos 0.0 .0 . sur $=100 \mathrm{vec} 1.0 .0$. dir $=1$. erg $=20$.

cut:p j .189

cut:e j .189

*f8:e,p $1234567891011121314151617181920 \mathrm{t}$

e8 0. 00000000120 .

fq0 $f e$

tf8 20

+fl8:e $1234567891011121314151617181920 \mathrm{t}$

c00. 1 .

f1:p $10019 \mathrm{i} 200$

f101:e $10019 \mathrm{i} 200$

*f11:p 10019 i 200

*f111:e 10019 i 200

f2:p $10019 i 200$

f102:e $10019 \mathrm{i} 200$

tf1 $5 \mathrm{j} 1$

tf101 5j 1

tf $115 \mathrm{j} 1$

tf111 5j 1

tf $25 \mathrm{j} 1$

tf102 5j 1

f4:e $118 \mathrm{i} 20$

sd4 1. 19r

tf4 20

*f14:e $118 \mathrm{i} 20$

sd14 1. 19r

tf14 20

m1 1000280001

$c$ Consider also estep $=15$

mode $p$ e [e]

imp:e $119 \mathrm{r} 0$

imp:p 1 19r 0

nps 1000000

ctme 120.

print

prdmp $2 \mathrm{j} 1$ 